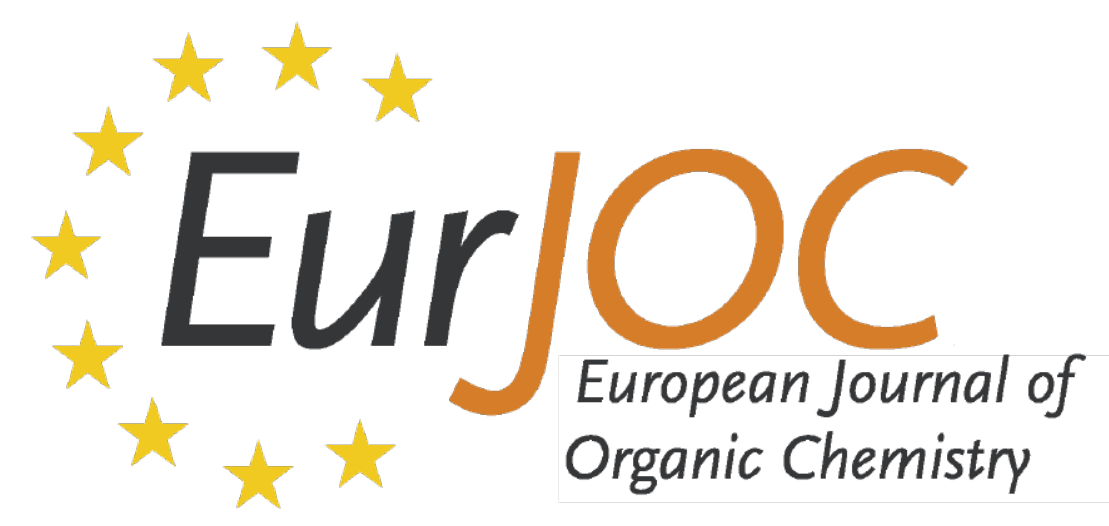

Supporting Information

\title{
Cobalt(III)-Catalyzed Direct ortho-Alkenylation of Arylpyrazoles: A Comparative Study on Decarboxylation and Desilylation
}

Anil Kumar, Nachimuthu Muniraj, and Kandikere Ramaiah Prabhu*

ejoc201900270-sup-0001-SupMat.pdf 
Table of contents

\begin{tabular}{|c|c|c|}
\hline S. No. & Title & Page No. \\
\hline 1 & General experimental & S-2 \\
\hline 2 & Detailed optimization studies & S-3 \\
\hline 3 & Scope for phenyl pyrazole derivatives with trimethylsilylacetylene & S-6 \\
\hline 4 & Unsuccessful substrates & S-9 \\
\hline 5 & Mechanistic studies & S-10 \\
\hline 6 & Characterization data for products & S-11 \\
\hline 7 & References & S-22 \\
\hline 8 & ${ }^{1} \mathrm{H}$ and ${ }^{13}$ C NMR spectra & S-23 \\
\hline
\end{tabular}




\section{General experimental}

General Information. All reactions were carried out using distilled solvents. Reactions were monitored by using precoated silica TLC plates. Mass spectra were recorded on EI, and ESI (TOF) modes. NMR spectra were recorded in at $400 \mathrm{MHz}$ spectrometers in $\mathrm{CDCl}_{3}, \mathrm{DMSO}_{6}$, tetramethylsilane (TMS; $\delta=$ $0.00 \mathrm{ppm}$ ) served as an internal standard for ${ }^{1} \mathrm{H}$ NMR. The corresponding residual non-deuterated solvent signal $\left(\mathrm{CDCl}_{3} ; \delta=77.00 \mathrm{ppm}\right.$ and DMSO-d 6 ; $\left.\delta=39.52 \mathrm{ppm}\right)$ was used as internal standard for ${ }^{13} \mathrm{C}$ NMR. Column chromatography was carried out on silica gel 230-400 mesh or 100-200 mesh (Merck) and thinlayer chromatography was carried out using SILICA GEL GF-254. Chemicals obtained from commercial suppliers were used without further purification. All arylpyrazoles derivatives, ${ }^{[1]}$ arylalkynyl carboxylic acid derivatives ${ }^{[2]}$, arylalkynyl silane derivatives, ${ }^{[3]}$ silver phenylacetylide, ${ }^{[4]}$ and cobalt catalysts ${ }^{[5]}$ were prepared according to reported literature procedure. 
Detailed optimization studies:

Table S1. Survey of the reaction parameters with $\operatorname{MnBr}(\mathrm{CO})_{5}$ catalyst. ${ }^{[\mathrm{a}, \mathrm{b}]}$

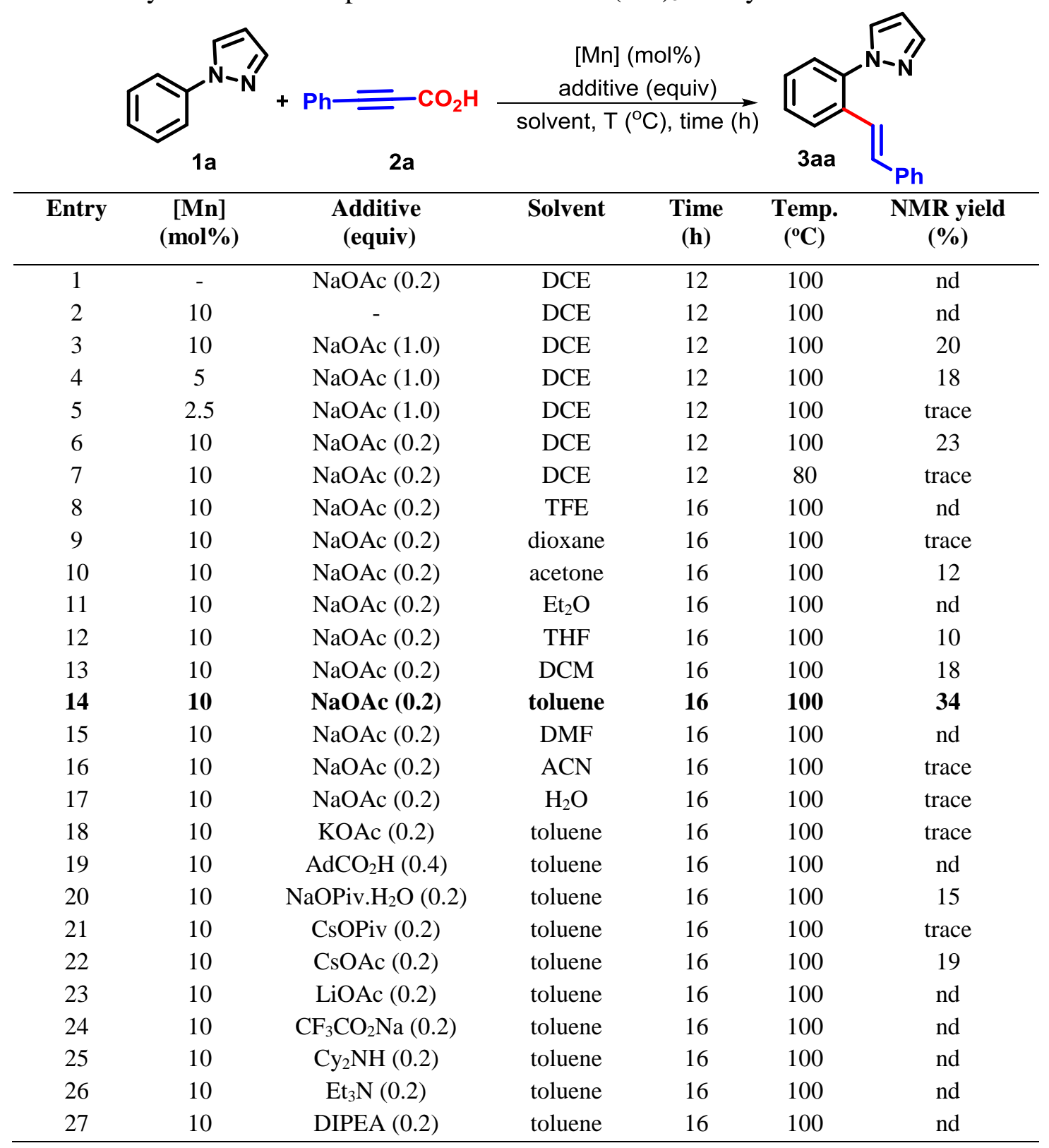

[a] Reaction conditions: 1a $(0.2 \mathrm{mmol}), \mathbf{2 a}(0.24 \mathrm{mmol}),[\mathrm{Mn}]=$ bromopentacarbonylmanganese $(\mathrm{I})$-catalyst $\mathrm{MnBr}(\mathrm{CO})_{5}(\mathrm{~mol} \%)$, additive (equiv), solvent $(2 \mathrm{~mL})$ at temperature $\left({ }^{\circ} \mathrm{C}\right)$ for given time. [b] ${ }^{1} \mathrm{H}$ NMR yield (using terepthalaldehyde as an internal standard). $\mathrm{AdCO}_{2} \mathrm{H}=1$-adamantane carboxylic acid. $\mathrm{nd}=$ not detected. 
Table S2. Survey of the reaction parameters with $\mathrm{Cp} * \mathrm{Co}(\mathrm{CO}) \mathrm{I}_{2}$ catalyst. ${ }^{[a, b]}$

\begin{tabular}{|c|c|c|c|c|c|c|}
\hline \multirow[b]{2}{*}{ Entry } & \multirow{2}{*}{$\begin{array}{c}1 \mathrm{a} \\
{[\mathrm{Co}]} \\
(5 \mathrm{~mol} \%)\end{array}$} & \multirow{2}{*}{$\begin{array}{c}+\mathrm{Ph}-\mathrm{SiMe}_{3} \\
\text { 2a' } \\
\begin{array}{c}\text { Additive } \\
\text { (equiv) }\end{array}\end{array}$} & \multicolumn{2}{|c|}{$\begin{array}{c}{[\mathrm{Co}](\mathrm{mol} \%)} \\
\underset{\mathrm{AgSbF}_{6}(20 \mathrm{~mol} \%)}{\stackrel{\text { additive }(\text { equiv })}{\longrightarrow}} \\
\text { solvent, } \mathrm{T}\left({ }^{\circ} \mathrm{C}\right) \text {, time }(\mathrm{h})\end{array}$} & 3aa & Ph \\
\hline & & & Solvent & $\begin{array}{c}\text { Time } \\
\text { (h) }\end{array}$ & $\begin{array}{c}\text { Temp. } \\
\left({ }^{\circ} \mathrm{C}\right)\end{array}$ & $\begin{array}{c}\text { NMR yield } \\
(\%)\end{array}$ \\
\hline 1 & 5 & $\mathrm{AdCO}_{2} \mathrm{H}(1.0)$ & DCE & 2 & 100 & 75 \\
\hline 2 & 5 & $\mathrm{NaOAc}(0.2)$ & DCE & 2 & 100 & 36 \\
\hline 3 & 5 & $\mathrm{CH}_{3} \mathrm{CO}_{2} \mathrm{H}(1.0)$ & TFE & 2 & 100 & 75 \\
\hline 4 & 5 & $\mathrm{CH}_{3} \mathrm{CO}_{2} \mathrm{H}(1.0)$ & TFE & 3 & 100 & 82 \\
\hline 5 & 5 & $\mathrm{CH}_{3} \mathrm{CO}_{2} \mathrm{H}(2.0) / \mathrm{CsF}(1.0)$ & TFE & 3 & 100 & 83 \\
\hline 6 & 5 & PTSA $(1.0)$ & TFE & 3 & 100 & 10 \\
\hline 7 & 5 & $\mathrm{CH}_{2}(\mathrm{COOH})_{2}(1.0)$ & TFE & 3 & 100 & 82 \\
\hline 8 & 5 & $\left(\mathrm{CH}_{3}\right)_{3} \mathrm{CCO}_{2} \mathrm{H}(2.0)$ & TFE & 3 & 100 & 77 \\
\hline 9 & 5 & $\mathrm{Cl}_{3} \mathrm{CCO}_{2} \mathrm{H}(2.0)$ & TFE & 3 & 100 & 83 \\
\hline 10 & 5 & $\mathrm{Cl}_{3} \mathrm{CCO}_{2} \mathrm{H}(1.0)$ & TFE & 3 & 100 & $86(83)^{c}$ \\
\hline 11 & 5 & $\mathrm{Cl}_{3} \mathrm{CCO}_{2} \mathrm{H}(0.2)$ & TFE & 3 & 100 & 47 \\
\hline 12 & 5 & $\mathrm{~F}_{3} \mathrm{CCO}_{2} \mathrm{H}(1.0)$ & TFE & 3 & 100 & 82 \\
\hline $13^{d}$ & $5[\mathrm{Ru}]$ & $\mathrm{Cl}_{3} \mathrm{CCO}_{2} \mathrm{H}(1.0)$ & TFE & 3 & 100 & 44 \\
\hline $14^{e}$ & 5 [Rh] & $\mathrm{Cl}_{3} \mathrm{CCO}_{2} \mathrm{H}(1.0)$ & TFE & 3 & 100 & 51 \\
\hline $15^{f}$ & $10[\mathrm{Mn}]$ & $\mathrm{Cl}_{3} \mathrm{CCO}_{2} \mathrm{H}(1.0)$ & TFE & 3 & 100 & nd \\
\hline
\end{tabular}

[a] Reaction conditions: 1a $(0.2 \mathrm{mmol}), \mathbf{2 a}(0.24 \mathrm{mmol})$, cobalt catalyst $[\mathrm{Co}]=\mathrm{Cp} * \mathrm{Co}(\mathrm{CO}) \mathrm{I}_{2}(5 \mathrm{~mol} \%), \mathrm{AgSbF}_{6}(20 \mathrm{~mol} \%)$, additive (equiv), solvent $\left(2 \mathrm{~mL}\right.$ ) at $100{ }^{\circ} \mathrm{C}$ for given time (h). [b] ${ }^{1} \mathrm{H}$ NMR yield (using terepthalaldehyde as an internal standard). [c] Isolated yield. [d] $5 \mathrm{~mol} \%$ of $\left[\mathrm{Ru}(p \text {-cymene }) \mathrm{Cl}_{2}\right]_{2}$ was used as a catalyst. [e] $5 \mathrm{~mol} \%$ of $\left[\mathrm{RhCp}^{*} \mathrm{Cl}_{2}\right]_{2}$ was used as a catalyst. [f] $10 \mathrm{~mol} \%$ of $\mathrm{MnBr}(\mathrm{CO})_{5}$ was used as a catalyst.

Table S3. Survey of the reaction parameters. ${ }^{[a, b]}$

\begin{tabular}{|c|c|c|c|c|c|c|}
\hline \multicolumn{2}{|r|}{$1 a$} & $=\mathrm{CO}_{2} \mathrm{H}$ & $\begin{array}{l}\text { [Co] }(5 \mathrm{~m} \\
\text { AgSbF }_{6} \\
\text { additive ( } \\
\text { solvent, }\end{array}$ & $\begin{array}{l}\text { \%) } \\
\text { mol\% } \\
\text { equiv) } \\
\text { C), tim }\end{array}$ & & baa \\
\hline Entry & $\begin{array}{c}{[\mathrm{Co}]} \\
(\mathrm{mol} \%)\end{array}$ & $\begin{array}{l}\text { Additive } \\
\text { (equiv) }\end{array}$ & Solvent & $\begin{array}{c}\text { Time } \\
\text { (h) }\end{array}$ & $\begin{array}{c}\text { Temp. } \\
\left({ }^{\circ} \mathrm{C}\right)\end{array}$ & $\begin{array}{c}\text { NMR yield } \\
(\%)\end{array}$ \\
\hline 1 & 5 & $\mathrm{NaOAc}(0.2)$ & TFE & 3 & 100 & 25 \\
\hline $2^{c}$ & 5 & $\mathrm{NaOAc}(0.2)$ & TFE & 3 & 100 & 18 \\
\hline 3 & 5 & NaOAc (0.2) & TFE & 3 & 120 & 25 \\
\hline 4 & 5 & $\mathrm{NaOAc}(1.0)$ & DCE & 3 & 100 & 20 \\
\hline 5 & 5 & $\mathrm{NaOH} \quad(1.0)$ & TFE & 3 & 100 & 15 \\
\hline
\end{tabular}

[a] Reaction conditions: $1 a(0.3 \mathrm{mmol}), \mathbf{5 a}(0.36 \mathrm{mmol})$, cobalt catalyst $[\mathrm{Co}]=\left[\mathrm{Cp} * \mathrm{Co}(\mathrm{CO}) \mathrm{I}_{2}\right](5 \mathrm{~mol} \%), \mathrm{AgSbF}_{6}(20 \mathrm{~mol} \%)$, additive (equiv), solvent ( $3 \mathrm{~mL}$ ) for $3 \mathrm{~h}$ at given temperature. [b] ${ }^{1} \mathrm{H}$ NMR yield (using terepthalaldehyde as an internal standard). [c] $0.6 \mathrm{mmol}$ of 5 was used. 
Table S4. Survey of the reaction parameters. ${ }^{[a, b]}$

\begin{tabular}{|c|c|c|c|c|c|c|}
\hline \multirow[b]{2}{*}{ Entry } & \multirow[b]{2}{*}{$\begin{array}{c}{[\mathrm{Co}]} \\
(\mathrm{mol} \%)\end{array}$} & \multirow[b]{2}{*}{$\begin{array}{l}\text { Additive } \\
\text { (equiv) }\end{array}$} & \multicolumn{2}{|c|}{$\begin{array}{c}{[\mathrm{Co}](5 \mathrm{~mol} \%)} \\
\underset{\mathrm{AgSbF}_{6}(20 \mathrm{~mol} \%)}{\stackrel{\text { Additive }(\mathrm{X} \text { equiv })}{\longrightarrow}} \\
\text { Solvent, } \mathrm{T}\left({ }^{\circ} \mathrm{C}\right), \text { Time }(\mathrm{h})\end{array}$} & 6aa & \multirow[b]{2}{*}{$\begin{array}{c}\text { NMR yield } \\
(\%)\end{array}$} \\
\hline & & & Solvent & $\begin{array}{c}\text { Time } \\
\text { (h) }\end{array}$ & $\begin{array}{c}\text { Temp. } \\
\left({ }^{\circ} \mathrm{C}\right)\end{array}$ & \\
\hline 1 & 5 & $\mathrm{CH}_{3} \mathrm{CO}_{2} \mathrm{H}(3.0)$ & TFE & 3 & 100 & 50 \\
\hline 2 & 5 & $\mathrm{CH}_{3} \mathrm{CO}_{2} \mathrm{H}(10.0)$ & TFE & 3 & 100 & 50 \\
\hline 3 & 5 & $\mathrm{Cl}_{3} \mathrm{CCO}_{2} \mathrm{H}(2.0)$ & TFE & 3 & 100 & 56 \\
\hline 4 & 5 & $\mathrm{Cl}_{3} \mathrm{CCO}_{2} \mathrm{H}(1.0)$ & TFE & 3 & 100 & $58(51 \%)^{c}$ \\
\hline 5 & 5 & $\mathrm{CH}_{3} \mathrm{CO}_{2} \mathrm{H}(2.4) / \mathrm{CsF}(1.2)$ & TFE & 3 & 100 & 27 \\
\hline 6 & 5 & $\mathrm{CCl}_{3} \mathrm{CO}_{2} \mathrm{H}(2.0)$ & TFE & 6 & 80 & 25 \\
\hline 7 & 5 & $\mathrm{CCl}_{3} \mathrm{CO}_{2} \mathrm{H}(2.0)$ & TFE & 6 & 120 & 54 \\
\hline 8 & 5 & $\mathrm{CCl}_{3} \mathrm{CO}_{2} \mathrm{H}(2.0)$ & TFE & 3 & 140 & 44 \\
\hline 9 & 10 & $\mathrm{Cl}_{3} \mathrm{CCO}_{2} \mathrm{H}(2.0)$ & TFE & 3 & 100 & 56 \\
\hline 10 & 5 & $\mathrm{Cl}_{3} \mathrm{CCO}_{2} \mathrm{H}(2.0)$ & TFE (degassed) & 3 & 100 & 56 \\
\hline 11 & 5 & $\mathrm{Cl}_{3} \mathrm{CCO}_{2} \mathrm{H}(2.0)$ & TFE $(0.5 \mathrm{~mL})$ & 3 & 100 & 52 \\
\hline 12 & 5 & $\mathrm{NH}_{4} \mathrm{Cl}(1.2)$ & TFE & 3 & 100 & nd \\
\hline 13 & 5 & $\mathrm{H}_{2} \mathrm{O}(5.0)$ & TFE & 3 & 100 & nd \\
\hline 14 & 5 & $\mathrm{~F}_{3} \mathrm{CCO}_{2} \mathrm{H}(2.0)$ & TFE & 3 & 100 & 52 \\
\hline 15 & 5 & none & TFE & 3 & 100 & 15 \\
\hline 16 & 5 & $\mathrm{AdCO}_{2} \mathrm{H}(2.0)$ & TFE & 3 & 100 & 40 \\
\hline 17 & 5 & $\mathrm{HCO}_{2} \mathrm{H}(2.0)$ & TFE & 3 & 100 & 52 \\
\hline 18 & 5 & $\mathrm{PhCO}_{2} \mathrm{H}(2.0)$ & TFE & 3 & 120 & 56 \\
\hline 19 & 5 & $\mathrm{CCl}_{3} \mathrm{CO}_{2} \mathrm{H}(2.0)$ & $\mathrm{THF}$ & 6 & 100 & 30 \\
\hline 20 & 5 & $\mathrm{Cl}_{3} \mathrm{CCO}_{2} \mathrm{H}(2.0)$ & toluene & 6 & 100 & 35 \\
\hline 21 & 5 & $\mathrm{Cl}_{3} \mathrm{CCO}_{2} \mathrm{H}(2.0)$ & $\mathrm{Et}_{2} \mathrm{O}$ & 6 & 100 & 30 \\
\hline 22 & 5 & $\mathrm{Cl}_{3} \mathrm{CCO}_{2} \mathrm{H}(2.0)$ & DMF & 6 & 100 & nd \\
\hline 23 & 5 & $\mathrm{Cl}_{3} \mathrm{CCO}_{2} \mathrm{H}(2.0)$ & DME & 3 & 100 & 10 \\
\hline 24 & 5 & $\mathrm{H} \equiv \mathrm{CO}_{2} \mathrm{H}(1.0)$ & TFE & 3 & 100 & traces \\
\hline
\end{tabular}

[a] Reaction conditions: 1a $(0.3 \mathrm{mmol}), \mathbf{5 a}(0.36 \mathrm{mmol})$, cobalt catalyst $[\mathrm{Co}]=\left[\mathrm{Cp} * \mathrm{Co}(\mathrm{CO}) \mathrm{I}_{2}\right](5 \mathrm{~mol} \%), \mathrm{AgSbF}_{6}(20 \mathrm{~mol} \%)$, Additive (equiv.), solvent $(2 \mathrm{~mL})$ at given time $(\mathrm{h})$ and temperature. [b] ${ }^{1} \mathrm{H}$ NMR yield (using terepthalaldehyde as an internal standard). [c] Isolated yield. nd = not detected. AdCOOH = 1-adamantane carboxylic acid. 
Scheme S1: Scope for 1-phenylpyrazole derivatives with trimethylsilylacetylene. ${ }^{[a, b]}$

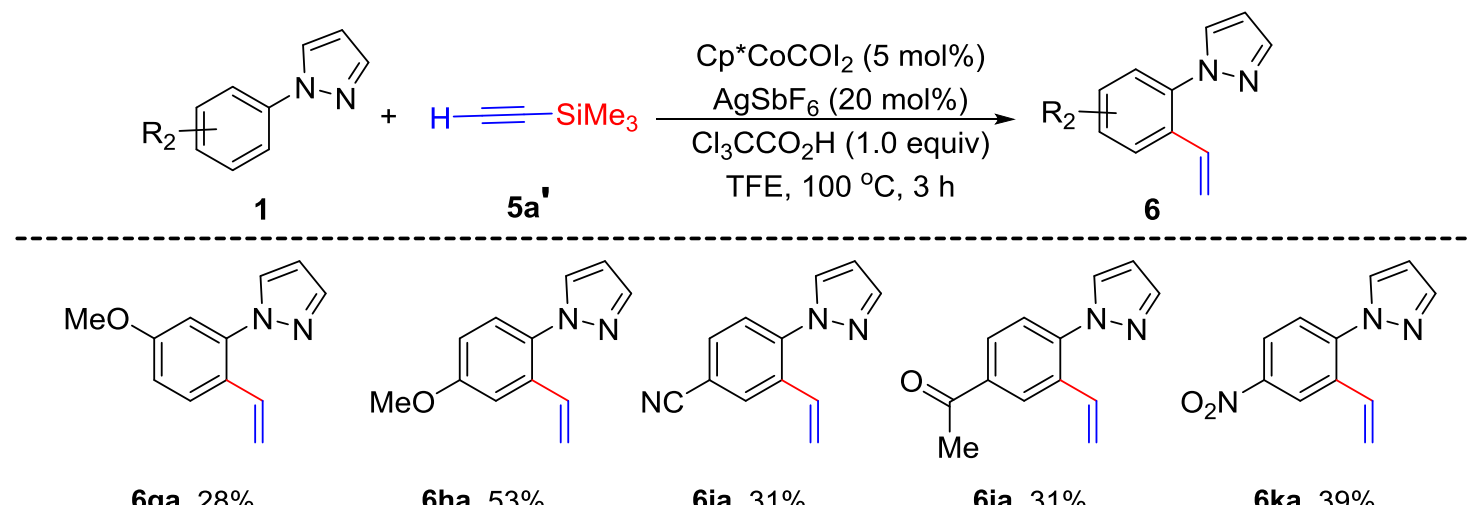

6ha, $53 \%$

6ia, 31\%

6ja, 31\%

6ka, 39\%

[a] Reaction conditions: $1(0.3 \mathrm{mmol}), \mathbf{5 a}(0.36 \mathrm{mmol}),\left[\mathrm{Cp} * \mathrm{Co}(\mathrm{CO}) \mathrm{I}_{2}\right](5 \mathrm{~mol} \%), \mathrm{AgSbF}_{6}(20 \mathrm{~mol} \%), \mathrm{Cl}_{3} \mathrm{CCO}_{2} \mathrm{H}(1.0$ equiv), TFE ( $3 \mathrm{~mL}$ ), at $100{ }^{\circ} \mathrm{C}$ for $3 \mathrm{~h}$. [b] ${ }^{1} \mathrm{H}$ NMR yields (measured by using terephthalaldehyde or 1,3,5-trimethoxybenzene as an internal standard).

The ortho-alkenylated products, 6ga-6ka, are inseparable mixture with corresponding 1-phenylpyrazole derivatives in both solvent system (ethyl acetate/petroleum ether or dichloromethane/petroleum ether). Therefore, crude sample of ${ }^{1} \mathrm{H}$ NMR spectras have been provided for $\mathbf{6 g a - 6 k a}$ molecules.

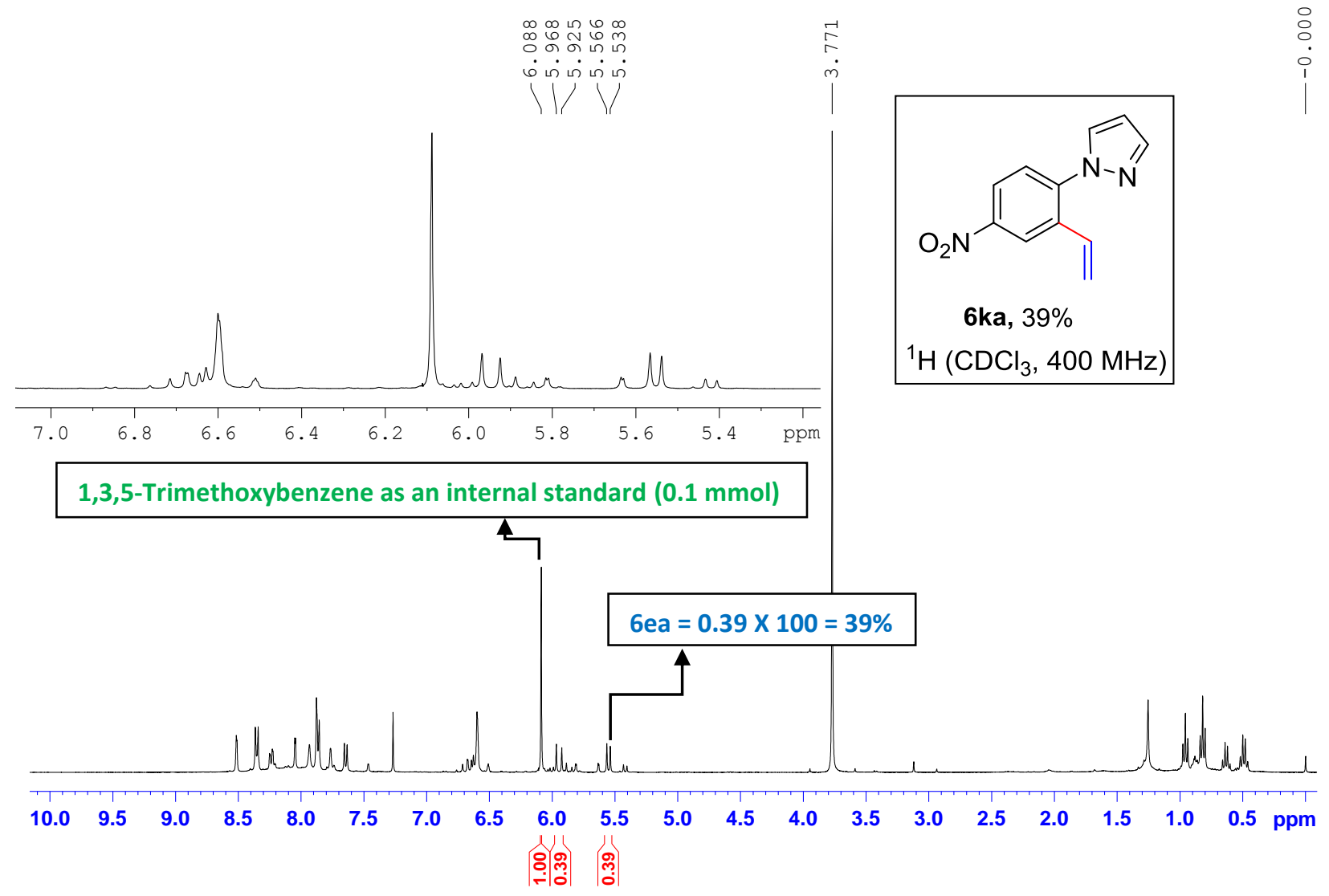




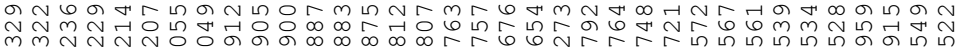

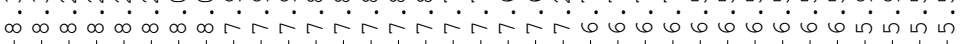
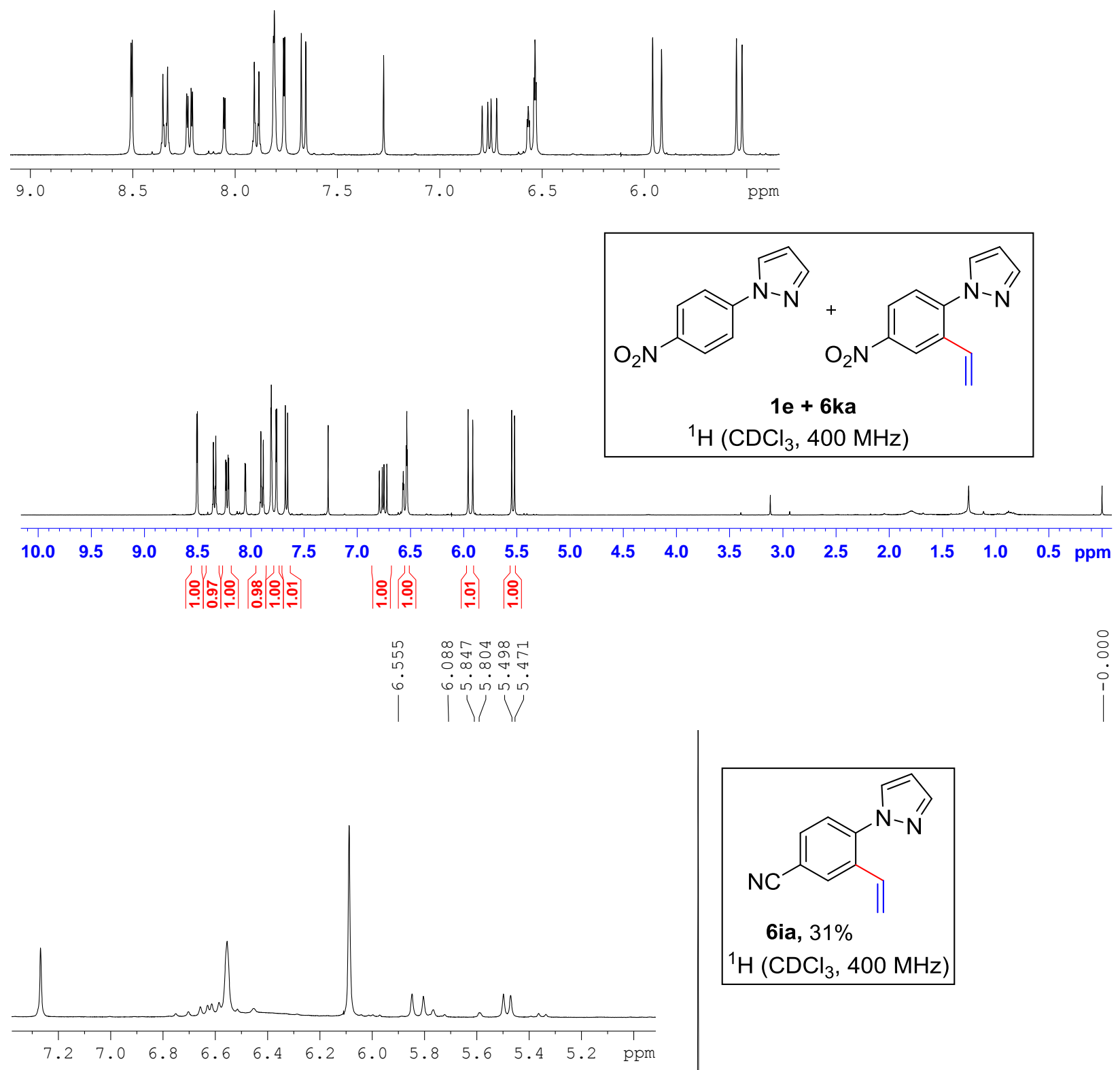

1,3,5-Trimethoxybenzene as an internal standard (0.1 $\mathrm{mmol})$

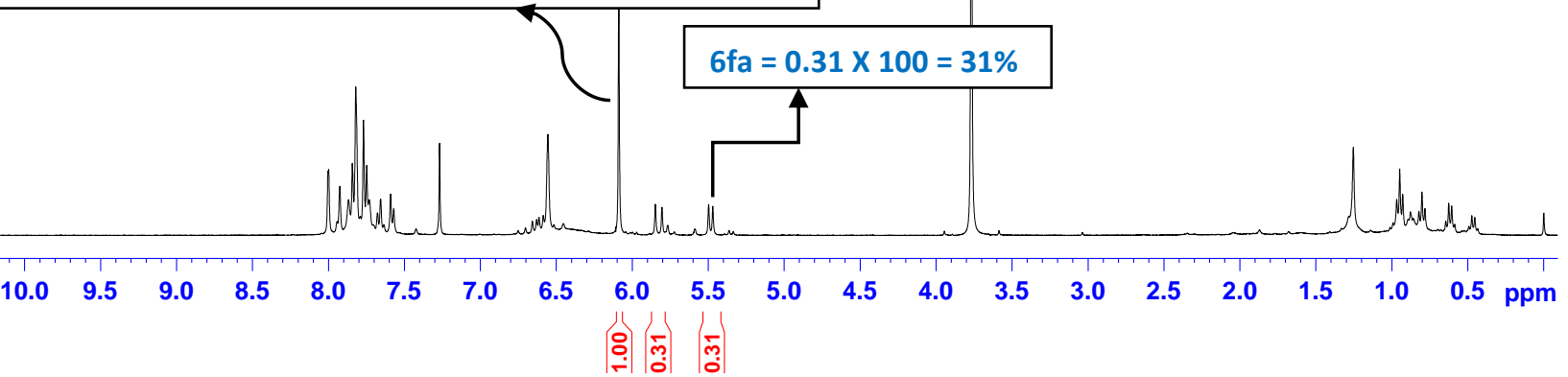



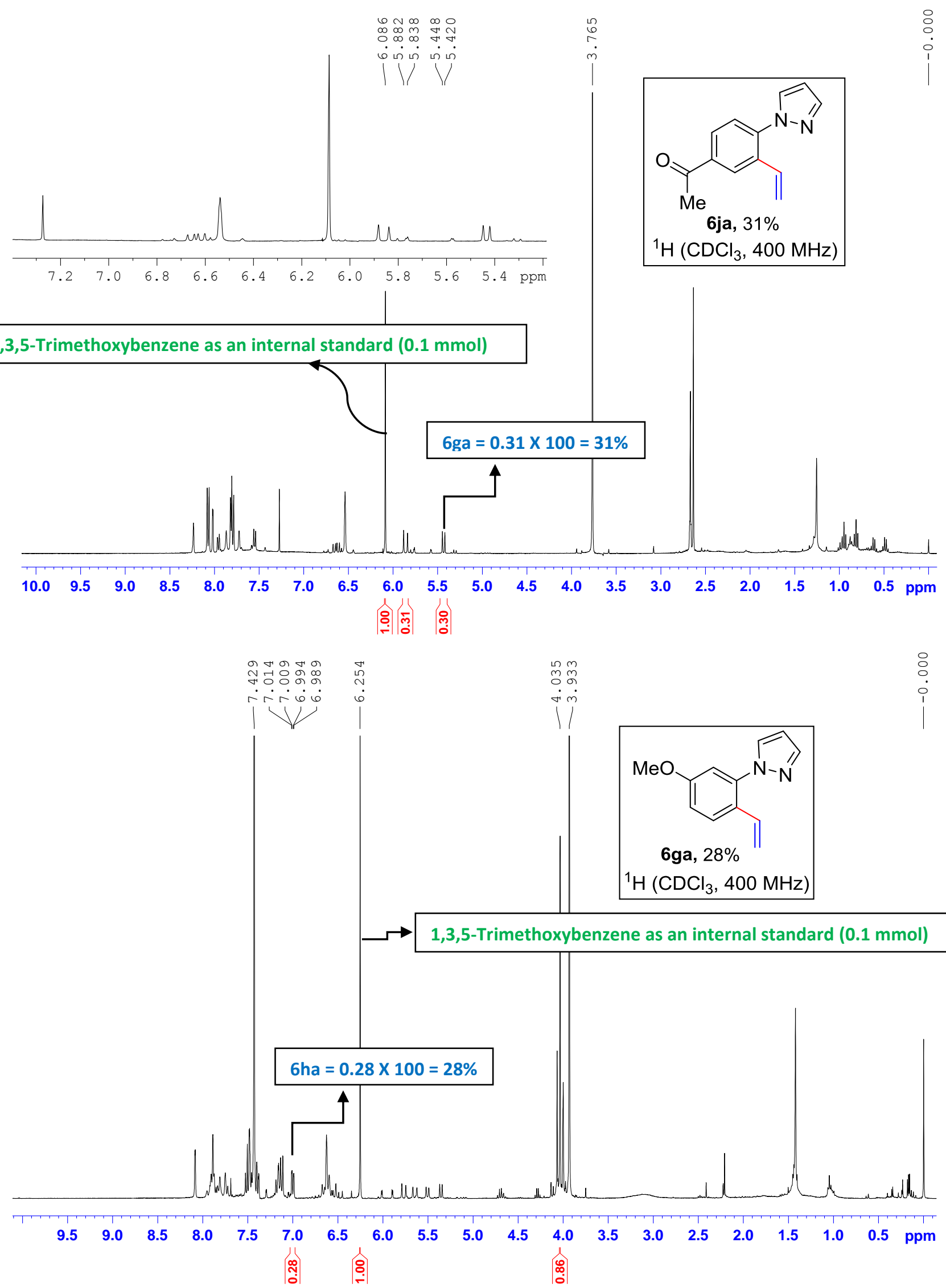


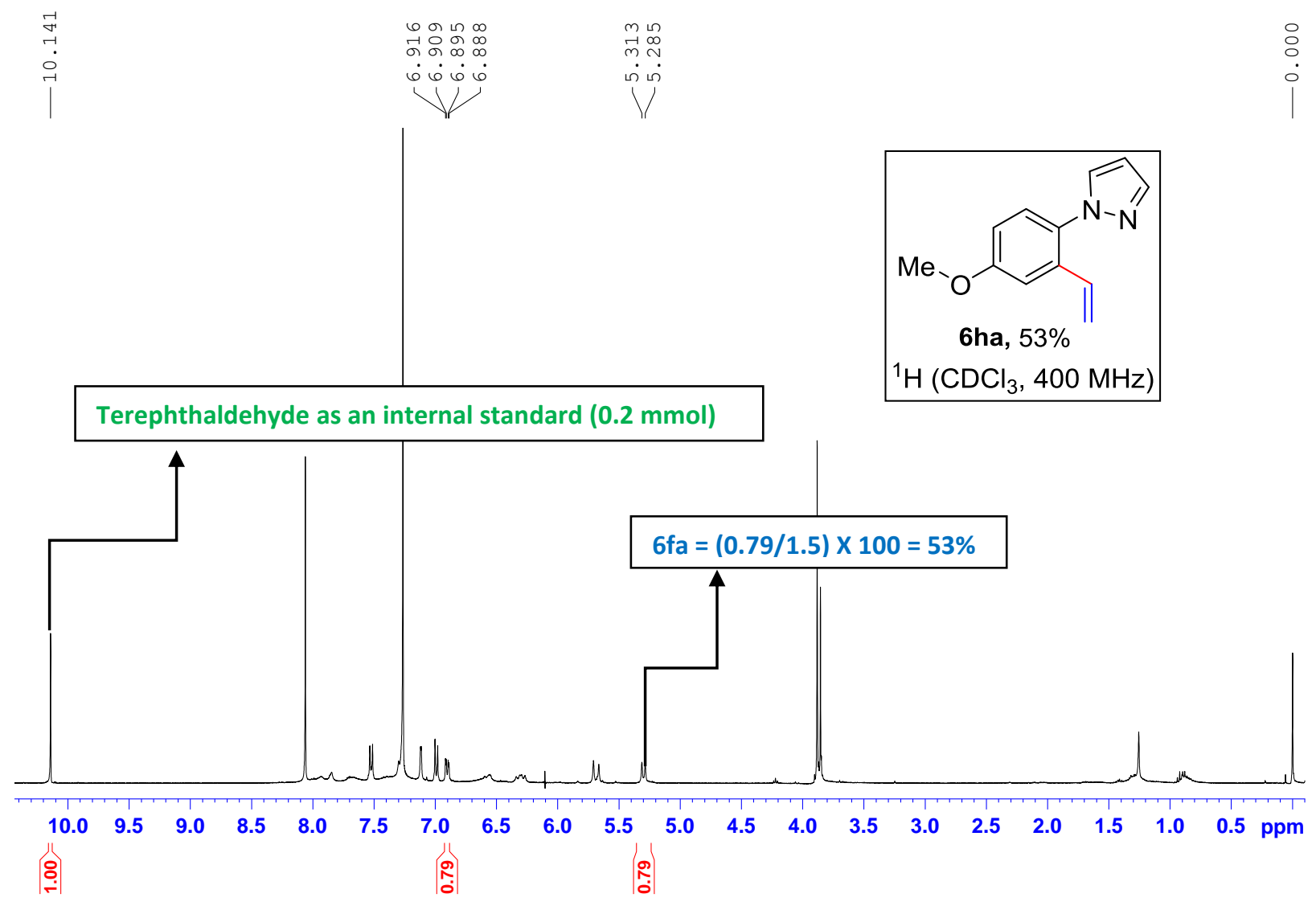

Scheme S2: Unsuccessful Substrates for ortho-alkenylation. ${ }^{[a]}$<smiles>C(=C/c1ncccc1-n1cccn1)\c1ccccc1</smiles><smiles>C(=C/c1ccccc1-c1ncccn1)\c1ccccc1</smiles><smiles>C(=C/c1cccn1-c1ncccn1)\c1ccccc1</smiles><smiles>C(=C/c1ccc2ccccc2c1-n1cccn1)\c1ccccc1</smiles><smiles>CC1=NN(c2ccccc2/C=C/C(C)=C\c2ccccc2)C(=O)C1</smiles><smiles>Sc1nnnn1-c1ccccc1/C=C/c1ccccc1</smiles><smiles>C=Cc1ccccc1-c1ccccn1</smiles><smiles>C=Cc1ccccc1-c1ncccn1</smiles><smiles>C=Cc1cccn1-c1ncccn1</smiles><smiles>C=Cc1ccc2ccccc2c1-n1cccn1</smiles><smiles>C=Cc1ccccc1N1N=C(C)CC1=O</smiles><smiles>C=Cc1ccccc1-n1nnnc1S</smiles>

[a] The desired ortho-alkenylated products were not detected. 


\section{Mechanistic studies}

\section{H/D-Exchange reaction}

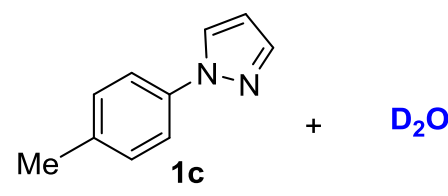

$(0.2 \mathrm{mmol})$

(10 equiv)

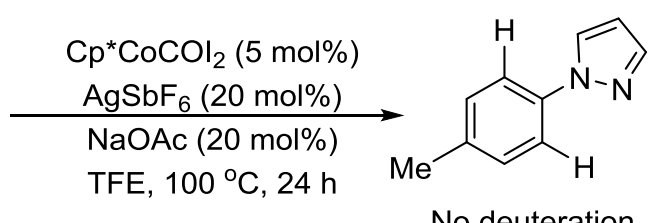<smiles>Cc1ccc(-n2cccn2)cc1</smiles>

$(10 \mathrm{mmol})$

(10 equiv)

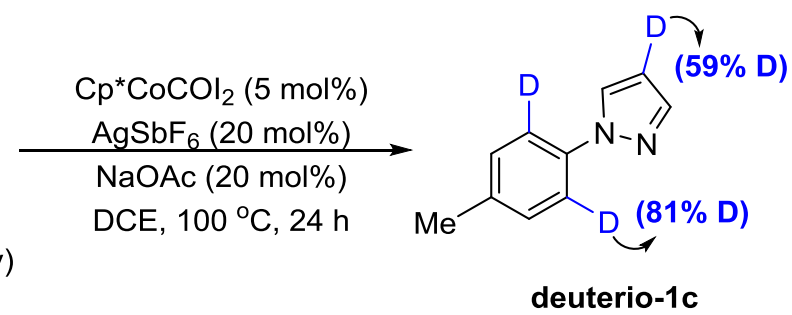

In a 8-mL screw cap reaction vial, 1-(4-methylphenyl)pyrazole 1c (160 mg, $10.0 \mathrm{mmol}), \mathrm{D}_{2} \mathrm{O}$ (10 equiv), cobalt catalyst $\mathrm{Cp} * \mathrm{CoCOI}_{2}(5 \mathrm{~mol} \%), \mathrm{NaOAc}(20 \mathrm{~mol} \%), \mathrm{AgSbF}_{6}(20 \mathrm{~mol} \%)$ were added followed by the addition of DCE $(6 \mathrm{~mL})$. This vial was sealed with a screw cap, placed on a pre-heated metal block at $100{ }^{\circ} \mathrm{C}$ for $24 \mathrm{~h}$ and cooled to room temperature and the crude product was purified by column chromatography on silica gel (EtOAc/petroleum ether, 1:1) to yield $81 \%$ deuterio-1c as a brown liquid (157 mg, 98\%). Deuterium incorporation was estimated by ${ }^{1} \mathrm{H}-\mathrm{NMR}$ analysis (See the following ${ }^{1} \mathrm{H}$ NMR spectra).

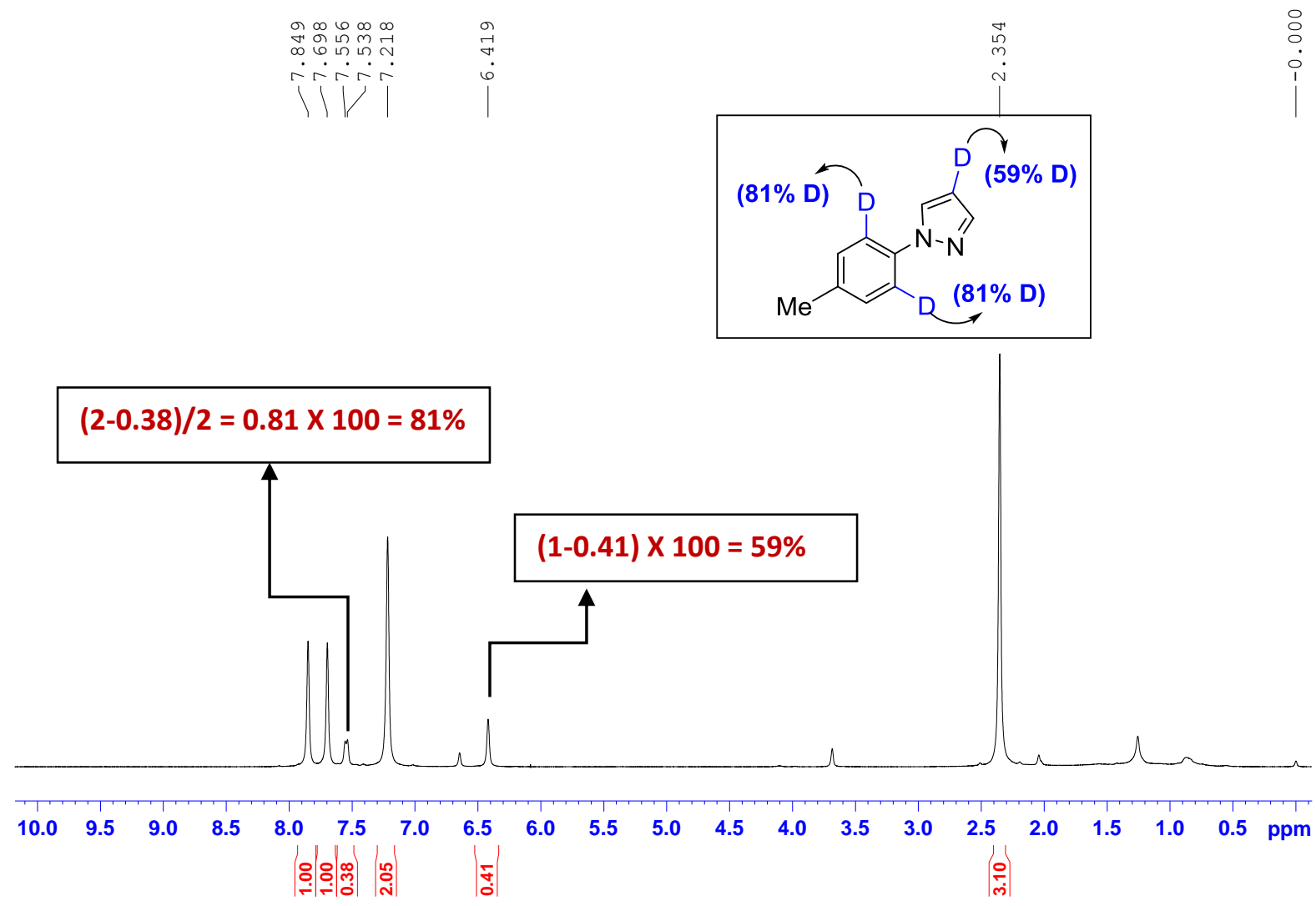




\section{Deuterium incorporation reaction}

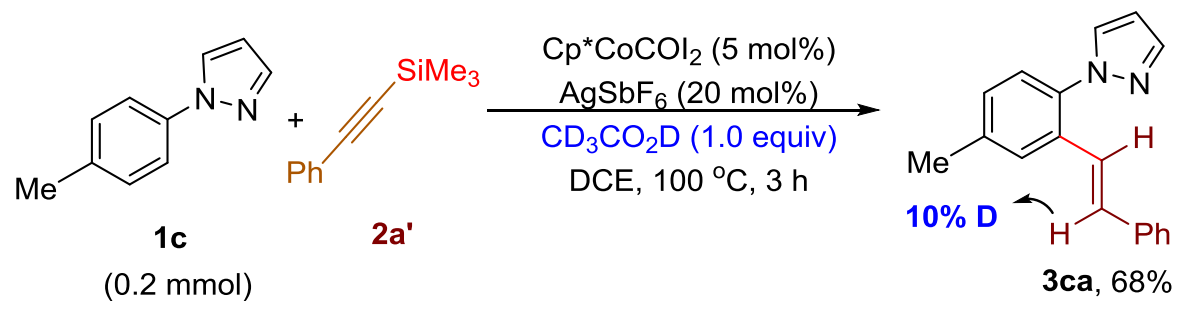

In a 8-mL screw cap reaction vial, 1-(4-methylphenyl)pyrazole 1c (32 mg, $0.2 \mathrm{mmol}), 2$ ' ' (42 mg, 0.24 mmol), cobalt catalyst $\mathrm{Cp}^{*} \mathrm{CoCOI}_{2}(4.8 \mathrm{mg}, 5 \mathrm{~mol} \%), \mathrm{CD}_{3} \mathrm{COOD}(12.0 \mathrm{mg}, 1.0 \mathrm{mmol}), \mathrm{AgSbF}_{6}(13.4$ $\mathrm{mg}, 20 \mathrm{~mol} \%)$ were added followed by the addition of DCE $(2 \mathrm{~mL})$. This vial was sealed with a screw cap, placed on a pre-heated metal block at $100{ }^{\circ} \mathrm{C}$ for $3 \mathrm{~h}$ and cooled to room temperature and concentrated to yield the crude product, which was further purified by flash chromatography (EtOAc/petroleum ether, 2:98) to furnish the product. The product was obtained with $10 \%$ of deuterium incorporation at the alkene carbon. The deuterium incorporation was calculated by ${ }^{1} \mathrm{H}$ NMR analysis (See the following ${ }^{1} \mathrm{H}$ NMR spectra).

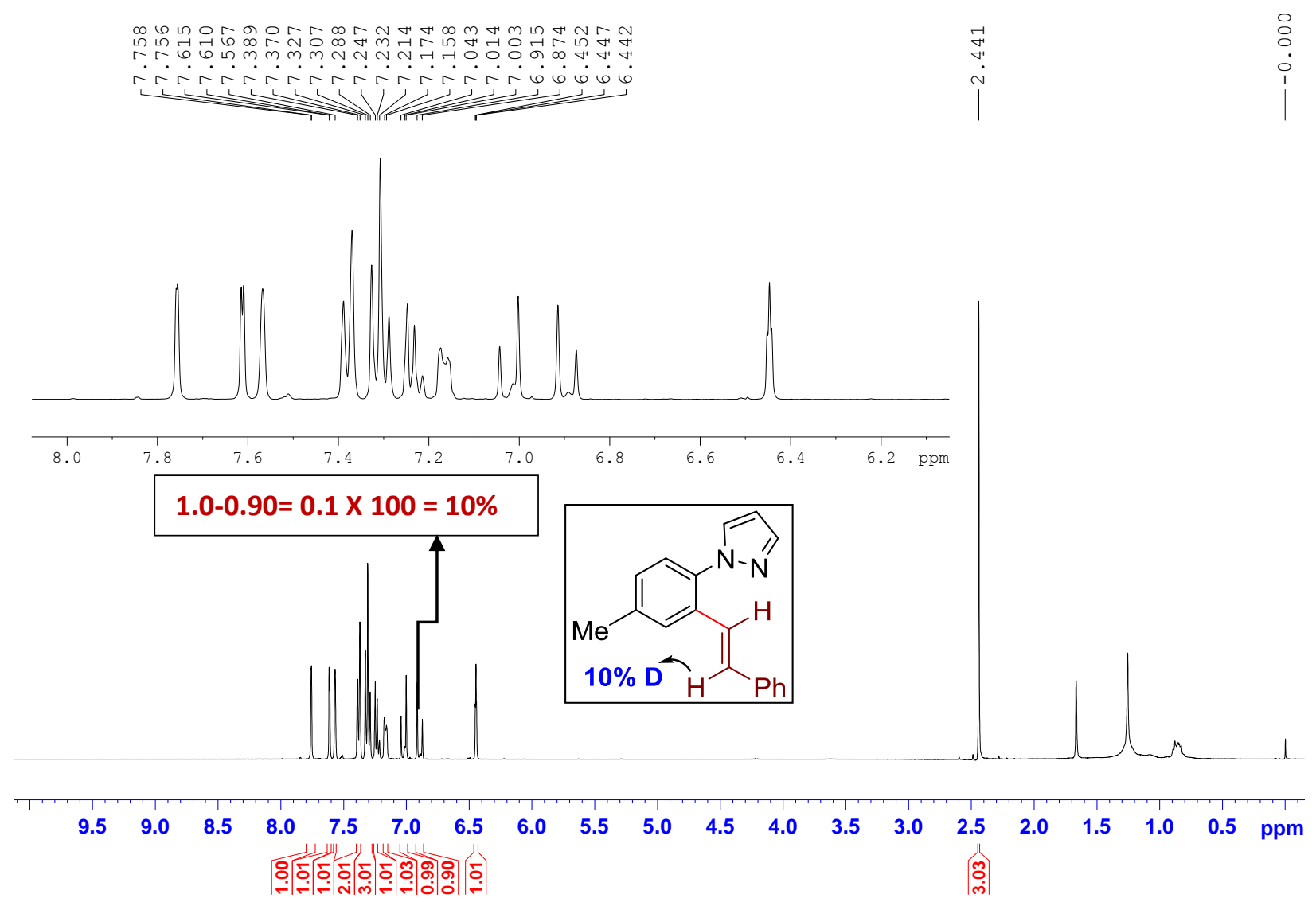




\section{$\underline{\text { Kinetic isotopic effect experiment }}$}

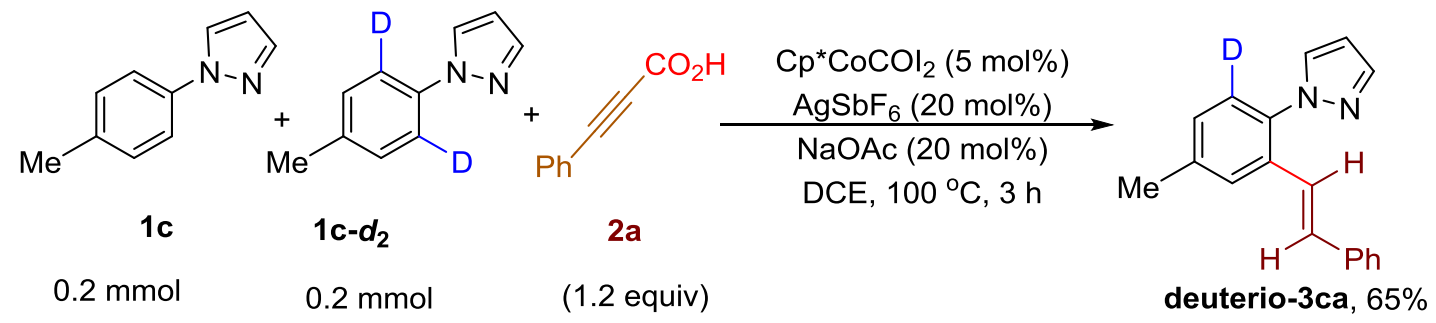

In an 8-mL screw cap reaction vial, 1:1 ratio of $\mathbf{1 a -} \boldsymbol{d}_{\mathbf{2}}(100 \% \mathrm{D})$ [ie. by weighing $0.25 \mathrm{mmol}$ of deuterio1c $81 \% \mathrm{D}$, which contains $0.2 \mathrm{mmol}$ of $\mathbf{1 a}-\boldsymbol{d}_{\mathbf{2}}(100 \% \mathrm{D})$ and $0.05 \mathrm{mmol}$ of $\left.\mathbf{1 a}\right]$ and $\mathbf{1 a}(0.15 \mathrm{mmol})$ were taken. (Now the reaction vial contains $0.2 \mathrm{mmol}$ of each of $\mathbf{1 a}$ and $\mathbf{1 a}-\boldsymbol{d}_{\mathbf{2}}(100 \% \mathrm{D})$. Then $\mathbf{2 a}(35 \mathrm{mg}, 0.24$ mmol), cobalt catalyst $\mathrm{Cp}^{*} \mathrm{CoCOI}_{2}$ (4.8 mg, $5 \mathrm{~mol} \%$ ), NaOAc (3.3 mg, $\left.20 \mathrm{~mol} \%\right), \mathrm{AgSbF}_{6}(13.4 \mathrm{mg}, 20$ mol \%) were added followed by the addition of DCE $(2 \mathrm{~mL})$. This vial was sealed with a screw cap, placed on a pre-heated metal block at $100{ }^{\circ} \mathrm{C}$ for $3 \mathrm{~h}$ and cooled to room temperature and concentrated to yield the crude product, which was further purified by flash chromatography (EtOAc/petroleum ether, 2:98) to furnish the product deuterio-3ca in $65 \%$ yield. The KIE was calculated from ${ }^{1} \mathrm{H}$ NMR analysis (See the following ${ }^{1} \mathrm{H}$ NMR spectra).

の

. . . . . . . . . . . . . . . . . .

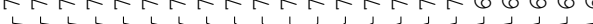

$\longrightarrow 1$

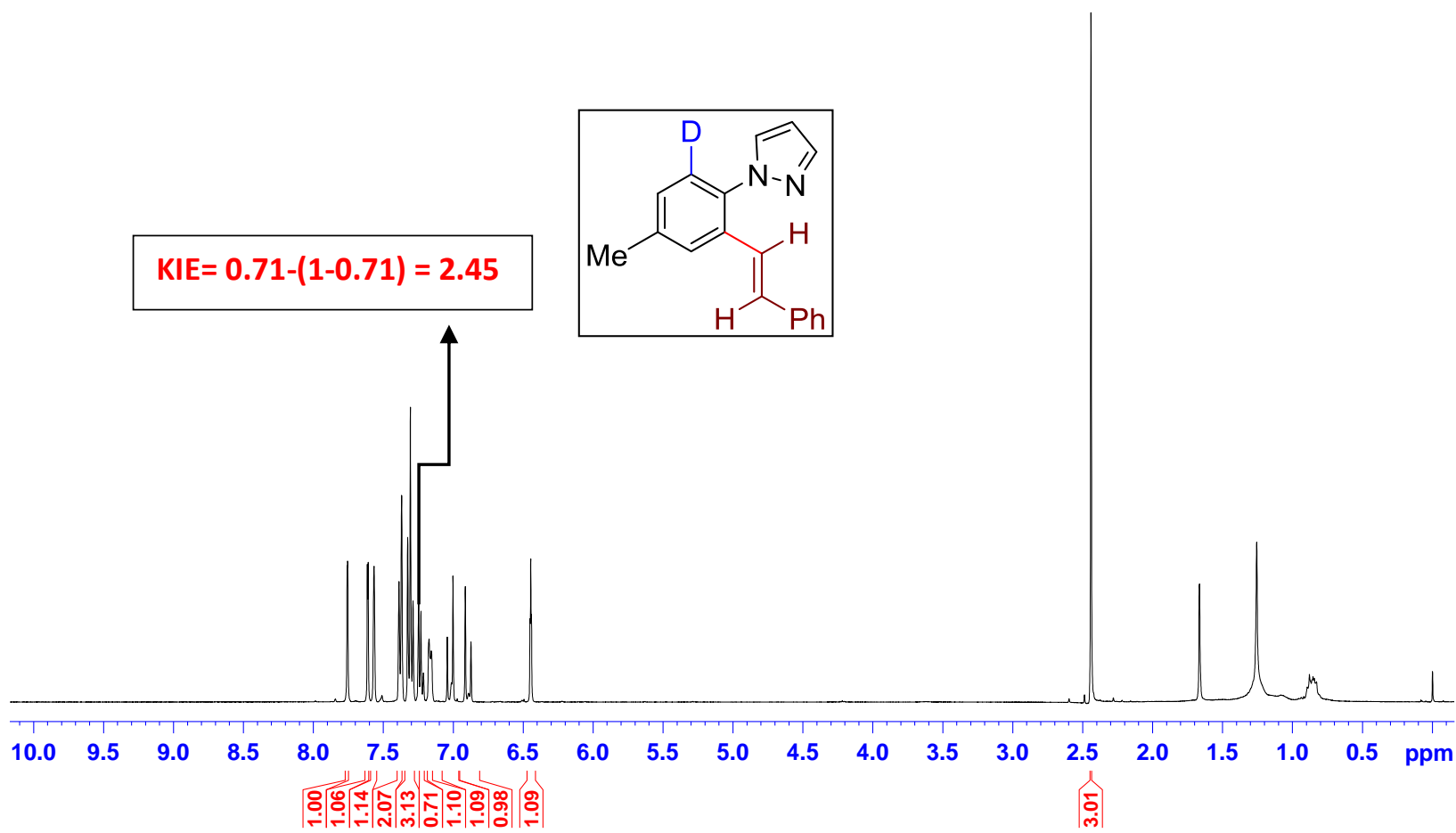




\section{Competitive experiment with aryl pyrazoles}
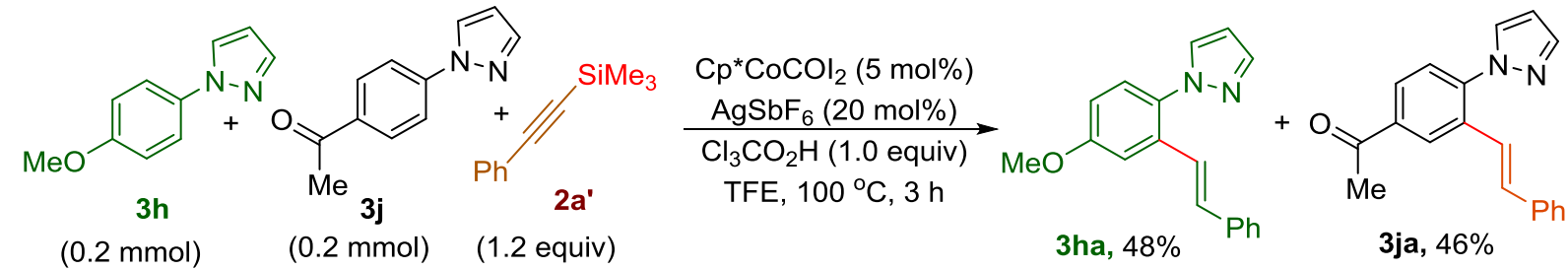

In a 8 -mL screw cap reaction vial, $\mathbf{3 h}(34.8 \mathrm{mg}, 0.2 \mathrm{mmol}), \mathbf{3 j}(37.2 \mathrm{mg}, 0.2 \mathrm{mmol}), \mathbf{2 a}(35.0 \mathrm{mg}, 0.24$ mmol), cobalt catalyst $\mathrm{Cp}^{*} \mathrm{CoCOI}_{2}(4.8 \mathrm{mg}, 5 \mathrm{~mol} \%), \mathrm{CD}_{3} \mathrm{COOD}$ (12.0 mg, 1.0 equiv), $\mathrm{AgSbF}_{6}(13.4$ $\mathrm{mg}, 20 \mathrm{~mol} \%)$ were added followed by the addition of TFE ( $2 \mathrm{~mL})$. This vial was sealed with a screw cap, placed on a pre-heated metal block at $100{ }^{\circ} \mathrm{C}$ for $3 \mathrm{~h}$, cooled to room temperature, filtered through a silica pad using a mixture of EtOAc and petroleum ether $(1: 1,100 \mathrm{~mL})$, and concentrated under vacuum. The crude products were submitted directly for ${ }^{1} \mathrm{H}$ NMR analysis for calculating the NMR yields (terephthalaldehyde $(0.1 \mathrm{mmol}, 13.4 \mathrm{mg})$ has been used as an internal standard, see the following ${ }^{1} \mathrm{H}$ NMR spectra). Aromatic protons were not assigned to individual products due to the intrinsic complexity of the spectrum.
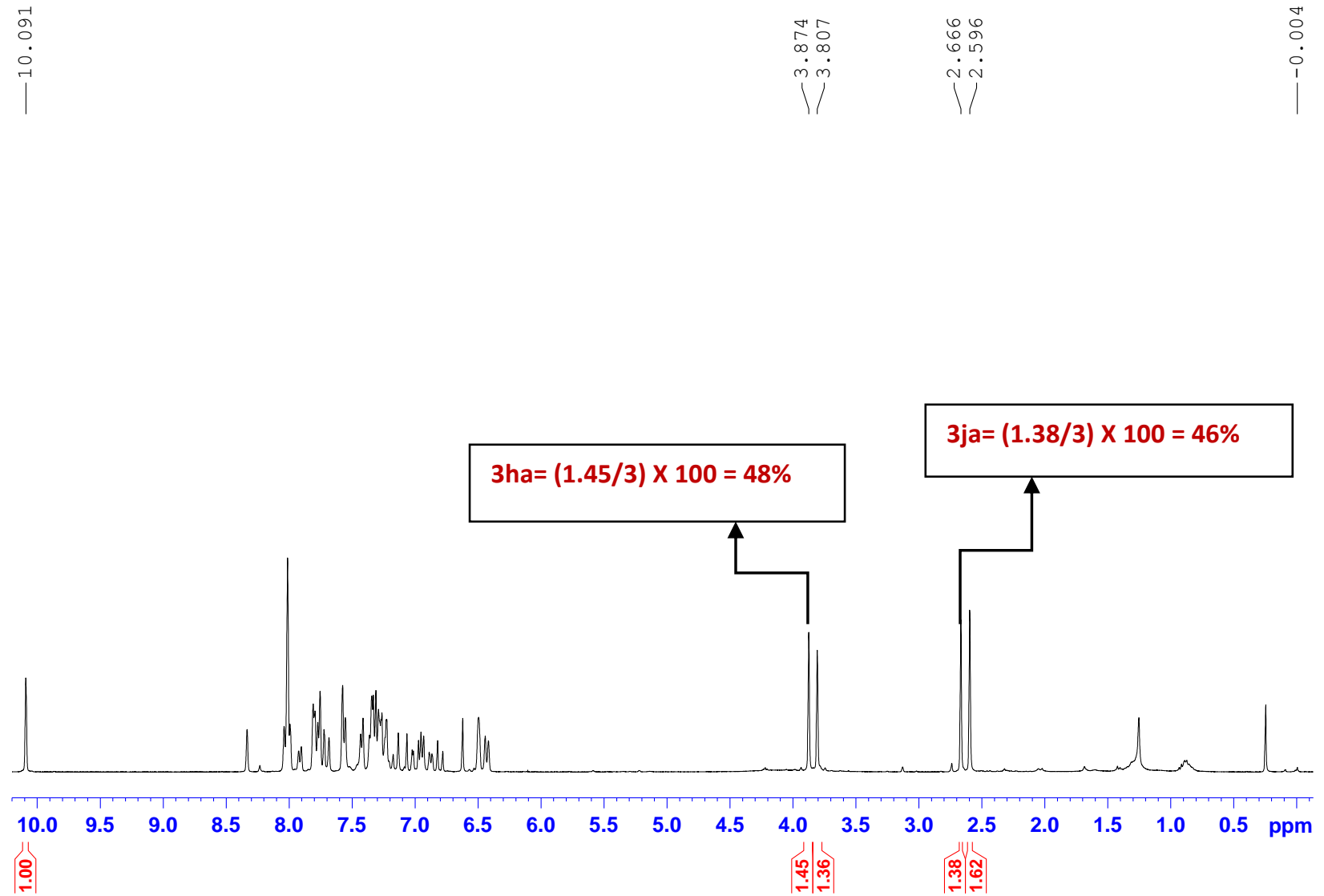

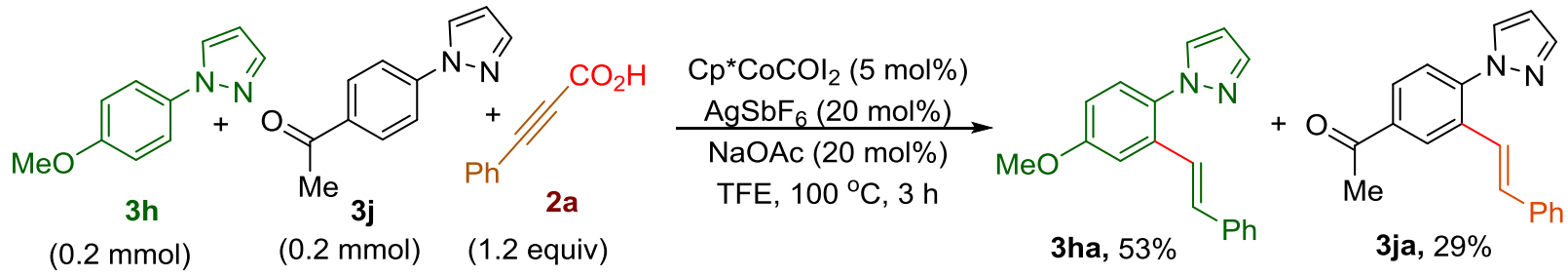

In a 8 -mL screw cap reaction vial, $\mathbf{3 h}(34.8 \mathrm{mg}, 0.2 \mathrm{mmol}), \mathbf{3 j}(37.2 \mathrm{mg}, 0.2 \mathrm{mmol}), \mathbf{2 a}(35.0 \mathrm{mg}, 0.24$ mmol), cobalt catalyst $\mathrm{Cp}^{*} \mathrm{CoCOI}_{2}(4.8 \mathrm{mg}, 5 \mathrm{~mol} \%), \mathrm{NaOAc}(3.3 \mathrm{mg}, 0.2 \mathrm{mmol}), \mathrm{AgSbF}_{6}(13.4 \mathrm{mg}, 20$ mol \%) were added followed by the addition of TFE $(2 \mathrm{~mL})$. This vial was sealed with a screw cap, placed on a pre-heated metal block at $100{ }^{\circ} \mathrm{C}$ for $3 \mathrm{~h}$ and cooled to room temperature filtered through a silica pad using a mixture of EtOAc and petroleum ether $(1: 1,100 \mathrm{~mL})$, and concentrated under vacuum. The crude products were submitted directly for ${ }^{1} \mathrm{H}$ NMR analysis for calculating the NMR yields (terephthalaldehyde $(0.1 \mathrm{mmol}, 13.4 \mathrm{mg})$ has been used as an internal standard, see the following ${ }^{1} \mathrm{H}$ NMR spectra). Aromatic protons were not assigned to individual products due to the intrinsic complexity of the spectrum.
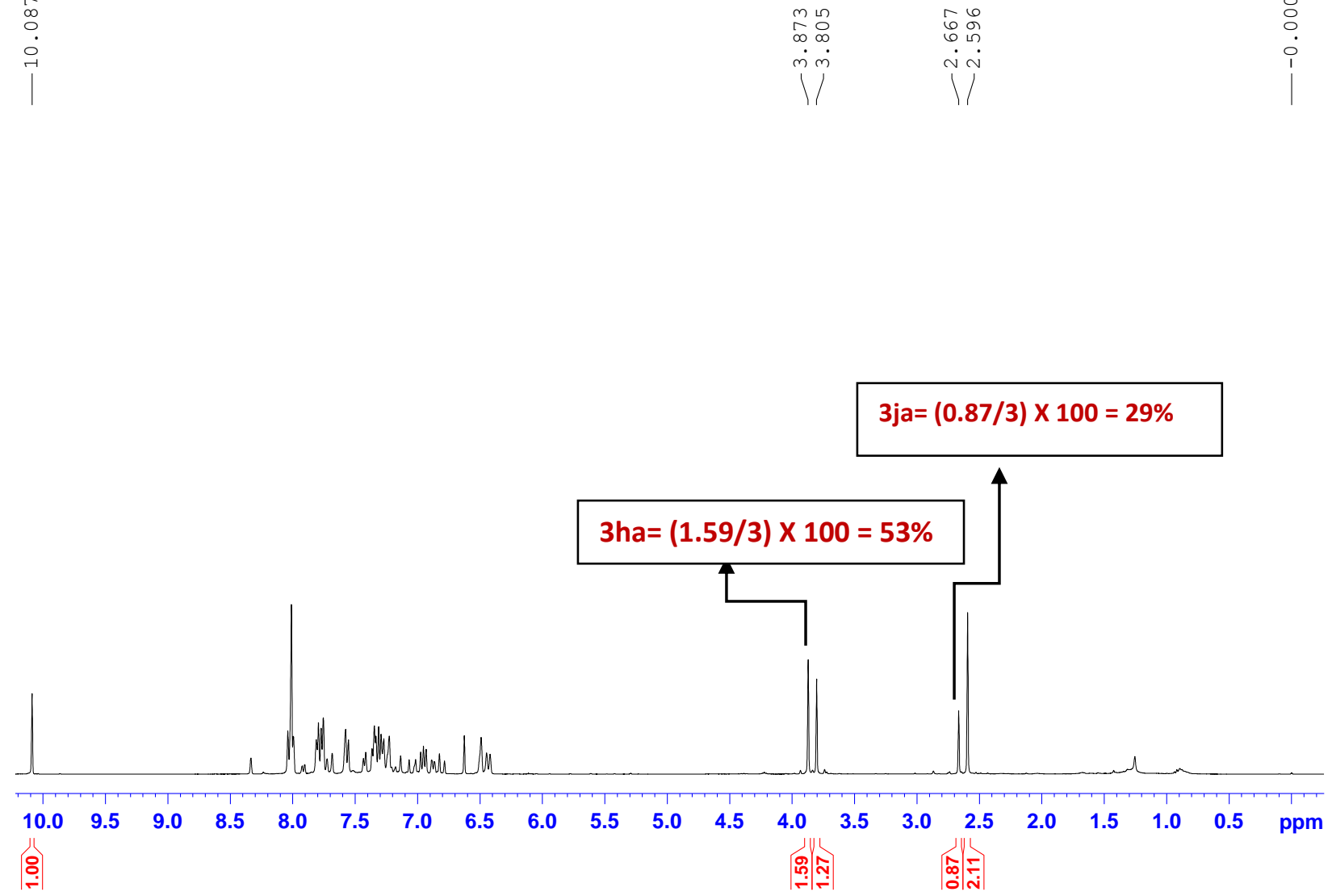


\section{Characterization data for products}

(E)-1-(2-Styrylphenyl)-1H-pyrazole (3aa). Colorless oil; Yield - (42.0 mg, 85\%); $\mathrm{R}_{\mathrm{f}}(10 \%$ EtOAc/Petroleum Ether) 0.50; Prepared as shown in general experimental procedure (a). IR $\left(\mathrm{KBr}, \mathrm{cm}^{-1}\right)$ : 3140, 3055, 3029, 2955, 2924, 2361, 2336, 1598, 1493,1458, 1392, 1328, 1218; ${ }^{1} \mathbf{H}$ NMR (400 MHz, CDCl $): \delta 6.55(\mathrm{t}, J=2.02 \mathrm{~Hz}, 1 \mathrm{H}) 7.02(\mathrm{~d}, J=16.28 \mathrm{~Hz}, 1 \mathrm{H}) 7.13(\mathrm{~d}, J=$ $16.28 \mathrm{~Hz}, 1 \mathrm{H}) 7.31-7.43(\mathrm{~m}, 3 \mathrm{H}) 7.45-7.54(\mathrm{~m}, 5 \mathrm{H}) 7.74(\mathrm{~d}, J=1.77 \mathrm{~Hz}, 1 \mathrm{H}) 7.84-7.86$ $(\mathrm{m}, 2 \mathrm{H}) ;{ }^{13} \mathrm{C}$ NMR $\left(100 \mathrm{MHz}, \mathrm{CDCl}_{3}\right) \delta 106.6,123.9,126.3,126.5,126.7,127.9,128.1,128.4$, 128.6, 131.2, 133.0, 137.0, 138.7, 140.7; HRESI-MS (mz): Calculated for $\mathrm{C}_{17} \mathrm{H}_{14} \mathrm{~N}_{2}(\mathrm{M}+\mathrm{Na})$ : 269.1055, found $(\mathrm{M}+\mathrm{Na}): 269.1058$.

(E)-1-(2-Methyl-6-styrylphenyl)-1H-pyrazole (3ba). Colorless oil; Yield - (38.0 mg, 73\%); $\mathbf{R}_{\mathbf{f}}$ (10\% EtOAc/Petroleum Ether) 0.50; Prepared as shown in general experimental procedure (a). IR $\left(\mathrm{KBr}, \mathrm{cm}^{-1}\right): 3052,3030,2956,2923,2853,2361,1594,1511,1470,1390,963$; ${ }^{1} \mathbf{H}$ NMR $\left(400 \mathrm{MHz}, \mathrm{CDCl}_{3}\right) \delta 2.05(\mathrm{~s}, 3 \mathrm{H}) 6.43(\mathrm{~d}, J=16.32 \mathrm{~Hz}, 1 \mathrm{H}) 6.48-6.49(\mathrm{~m}, 1 \mathrm{H}) 6.97(\mathrm{~d}, J=$ $16.32 \mathrm{~Hz}, 1 \mathrm{H}) 7.21-7.23(\mathrm{~m}, 2 \mathrm{H}) 7.26-7.31(\mathrm{~m}, 4 \mathrm{H}) 7.36(\mathrm{t}, J=7.64 \mathrm{~Hz}, 1 \mathrm{H}) 7.50(\mathrm{~d}, J=$ $2.02 \mathrm{~Hz}, 1 \mathrm{H}) 7.62(\mathrm{~d}, J=7.58 \mathrm{~Hz}, 1 \mathrm{H}) 7.79-7.80(\mathrm{~m}, 1 \mathrm{H}) ;{ }^{13} \mathbf{C ~ N M R}\left(100 \mathrm{MHz}, \mathrm{CDCl}_{3}\right): \delta$ 17.4, 106.2, 123.3, 126.6, 127.9, 128.6, 129.2, 129.7, 131.2, 131.8, 135.8, 136.7, 137.0, 138.0, 140.4; HRESI-MS (mz): Calculated for $\mathrm{C}_{18} \mathrm{H}_{16} \mathrm{~N}_{2}(\mathrm{M}+\mathrm{Na})$ : 283.1211 , found $(\mathrm{M}+\mathrm{Na})$ : 283.1215 .

(E)-1-(4-Methyl-6-styrylphenyl)-1H-pyrazole (3ca). Colorless oil; Yield - (48.0 mg, 92\%); $\mathbf{R}_{\mathrm{f}}$ (20\% EtOAc/Petroleum Ether) 0.60; Prepared as shown in general experimental procedure (a). IR $\left(\mathrm{KBr}, \mathrm{cm}^{-1}\right): 3107,3052,3028,2955,2923,2855,2361,2336,1724,1514,1393,963 ;{ }^{1} \mathbf{H}$ NMR (400 MHz, $\left.\mathrm{CDCl}_{3}\right) \delta 2.45(\mathrm{~s}, 3 \mathrm{H}) 6.45(\mathrm{t}, J=1.92 \mathrm{~Hz}, 1 \mathrm{H}) 6.89(\mathrm{~d}, J=16.28 \mathrm{~Hz}, 1 \mathrm{H})$ $7.03(\mathrm{~d}, J=16.28 \mathrm{~Hz}, 1 \mathrm{H}) 7.17(\mathrm{~d}, J=8.0 \mathrm{~Hz} 1 \mathrm{H}) 7.22-7.25(\mathrm{~m}, 1 \mathrm{H}) 7.29-7.33(\mathrm{~m}, 3 \mathrm{H}) 7.38$ $(\mathrm{d}, J=7.32 \mathrm{~Hz}, 2 \mathrm{H}) 7.57(\mathrm{~s}, 1 \mathrm{H}) 7.62(\mathrm{~d}, J=2.16 \mathrm{~Hz}, 1 \mathrm{H}) 7.76-7.77(\mathrm{~m}, 1 \mathrm{H}) ;{ }^{13} \mathbf{C}$ NMR $(100$ $\left.\mathrm{MHz}_{2} \mathrm{CDCl}_{3}\right) \delta 21.2,106.4,123.9,126.2,126.6,126.8,127.8,128.6,128.9,130.9,131.6,132.7$, 136.5, 137.1, 138.3, 140.5; HRESI-MS (mz): Calculated for $\mathrm{C}_{18} \mathrm{H}_{16} \mathrm{~N}_{2}(\mathrm{M}+\mathrm{Na})$ : 283.1211, found $(\mathrm{M}+\mathrm{Na}): 283.1209$.

(E)-1-(5-Chloro-2-styrylphenyl)-1H-pyrazole (3da). Dark yellow oil; Yield - (40.0 mg, 71\%); $\mathrm{R}_{\mathrm{f}}(20 \%$ EtOAc/Petroleum Ether) 0.45; Prepared as shown in general experimental procedure (a). IR $\left(\mathrm{KBr}, \mathrm{cm}^{-1}\right): 3057,3027,2923,2851,2362,2336,1594,1514,1493,1399 ;{ }^{1} \mathbf{H}$ NMR (400 $\left.\mathrm{MHz}, \mathrm{CDCl}_{3}\right) \delta 6.48(\mathrm{t}, J=2.15 \mathrm{~Hz}, 1 \mathrm{H}) 6.91(\mathrm{~d}, J=16.28 \mathrm{~Hz}, 1 \mathrm{H}) 7.03(\mathrm{~d}, J=16.28 \mathrm{~Hz}, 1 \mathrm{H})$ $7.22-7.27(\mathrm{~m}, 1 \mathrm{H}) 7.32(\mathrm{t}, J=7.33 \mathrm{~Hz}, 2 \mathrm{H}) 7.37-7.40(\mathrm{~m}, 3 \mathrm{H}) 7.49(\mathrm{~d}, J=2.27 \mathrm{~Hz}, 1 \mathrm{H})$ $7.66-7.70(\mathrm{~m}, 2 \mathrm{H}) 7.78(\mathrm{~d}, J=1.77 \mathrm{~Hz}, 1 \mathrm{H}) ;{ }^{13} \mathbf{C} \mathbf{N M R}\left(100 \mathrm{MHz}, \mathrm{CDCl}_{3}\right) \delta$ 107.0, 122.9, $126.3,126.7,127.7,128.2,128.5,128.7,131.3,131.5,131.7,133.4,136.7,139.3,141.2$; HRESI-MS $(m z)$ : Calculated for $\mathrm{C}_{17} \mathrm{H}_{13} \mathrm{~N}_{2} \mathrm{Cl}(\mathrm{M}+\mathrm{Na})$ : 303.0665 , found $(\mathrm{M}+\mathrm{Na}): 303.0669$. 
(E)-1-(2-Fluoro-6-styrylphenyl)-1H-pyrazole (3ea). Light yellow solid; Yield - (43.5 mg, 82\%); $\boldsymbol{m p}$ : $114-116{ }^{\circ} \mathrm{C} ; \mathrm{R}_{\mathrm{f}}(10 \%$ EtOAc/Petroleum Ether) 0.4; Prepared as shown in general experimental procedure (a). IR $\left(\mathrm{KBr}, \mathrm{cm}^{-1}\right): 3053,3028,2923,2852,2361,1608,1578,1476$, 1390, 1237; ${ }^{1} \mathbf{H}$ NMR $\left(400 \mathrm{MHz}, \mathrm{CDCl}_{3}\right) \delta 6.51(\mathrm{~s}, 1 \mathrm{H}) 6.68(\mathrm{~d}, J=16.36 \mathrm{~Hz}, 1 \mathrm{H}) 7.03(\mathrm{~d}, J=$ $16.36 \mathrm{~Hz}, 1 \mathrm{H}) 7.12(\mathrm{t}, J=8.72 \mathrm{~Hz}, 1 \mathrm{H}) 7.22-7.25(\mathrm{~m}, 1 \mathrm{H}) 7.28-7.35(\mathrm{~m}, 4 \mathrm{H}) 7.37-7.42$ $(\mathrm{m}, 1 \mathrm{H}) 7.56-7.61(\mathrm{~m}, 2 \mathrm{H}) 7.83(\mathrm{~s}, 1 \mathrm{H}) ;{ }^{13} \mathbf{C} \mathbf{~ N M R}\left(100 \mathrm{MHz}, \mathrm{CDCl}_{3}\right) \delta 106.7,114.8\left(J_{C-F}=\right.$ $20.4 \mathrm{~Hz}), 121.3\left(J_{C-F}=3.4 \mathrm{~Hz}\right), 122.3\left(J_{C-F}=3.2 \mathrm{~Hz}\right), 126.7,126.8,128.2,128.6,129.8\left(J_{C-F}=\right.$ $8.6 \mathrm{~Hz}), 132.5\left(J_{C-F}=14.4 \mathrm{~Hz}\right), 136.6,137.5,141.1,157.9\left(J_{C-F}=249.5 \mathrm{~Hz}\right) ;$ HRESI-MS $(\mathrm{mz})$ : Calculated for $\mathrm{C}_{17} \mathrm{H}_{13} \mathrm{~N}_{2} \mathrm{~F}(\mathrm{M}+\mathrm{Na})$ : 287.0960, found $(\mathrm{M}+\mathrm{Na})$ : 287.0960.

(E)-1-(4-Fluoro-2-styrylphenyl)-1H-pyrazole (3fa). Light brown solid; Yield - (46.5 mg, 88\%); $\boldsymbol{m p}$ : $66-68{ }^{\circ} \mathrm{C} ; \mathrm{R}_{\mathrm{f}}(10 \%$ EtOAc/Petroleum Ether) 0.5; Prepared as shown in general experimental procedure (a). IR $\left(\mathrm{KBr}, \mathrm{cm}^{-1}\right): 3058,2921,2851,2361,2357,1636,1610,1585$, 1496, 1394, 1268, 966; ${ }^{1} \mathbf{H}$ NMR $\left(400 \mathrm{MHz}, \mathrm{CDCl}_{3}\right) \delta 6.48(\mathrm{t}, J=2.15 \mathrm{~Hz}, 1 \mathrm{H}) 6.82(\mathrm{dd}, J=$ 16.04, $1.26 \mathrm{~Hz}, 1 \mathrm{H}) 7.02-7.08(\mathrm{~m}, 2 \mathrm{H}) 7.25-7.42(\mathrm{~m}, 6 \mathrm{H}) 7.45(\mathrm{dd}, J=9.85,2.78 \mathrm{~Hz}, 1 \mathrm{H})$ $7.61(\mathrm{~d}, J=2.02 \mathrm{~Hz}, 1 \mathrm{H}) 7.77(\mathrm{~d}, J=1.52 \mathrm{~Hz}, 1 \mathrm{H}) ;{ }^{13} \mathbf{C} \mathbf{N M R}\left(100 \mathrm{MHz}, \mathrm{CDCl}_{3}\right) \delta 106.7$, $112.6\left(J_{C-F}=23.2 \mathrm{~Hz}\right), 114.9\left(J_{C-F}=23.1 \mathrm{~Hz}\right), 122.7,126.8,128.3\left(J_{C-F}=4.3 \mathrm{~Hz}\right), 128.4\left(J_{C-F}=\right.$ $4.6 \mathrm{~Hz}), 128.7,131.7,132.4,134.9,135.5\left(J_{C-F}=8.3 \mathrm{~Hz}\right), 136.5,140.8,162.3\left(J_{C-F}=246 \mathrm{~Hz}\right)$; HRESI-MS $(m z)$ : Calculated for $\mathrm{C}_{17} \mathrm{H}_{13} \mathrm{~N}_{2} \mathrm{~F}(\mathrm{M}+\mathrm{Na})$ : 287.0960, found $(\mathrm{M}+\mathrm{Na})$ : 287.0961 .

(E)-1-(5-Methoxy-2-styrylphenyl)-1H-pyrazole (3ga). Light yellow oil; Yield - (26.0 mg, 47\%); $\mathrm{R}_{\mathrm{f}}(20 \% \mathrm{EtOAc/Petroleum} \mathrm{Ether)} \mathrm{0.45;} \mathrm{Prepared} \mathrm{as} \mathrm{shown} \mathrm{in} \mathrm{general} \mathrm{experimental}$ procedure (a). IR $\left(\mathrm{KBr}, \mathrm{cm}^{-1}\right): 3055,2928,2841,2361,2336,1604,1513,1289,1044 ;{ }^{1} \mathbf{H}$ NMR $\left(400 \mathrm{MHz}, \mathrm{CDCl}_{3}\right) \delta 3.84(\mathrm{~s}, 3 \mathrm{H}) 6.47(\mathrm{~s}, 1 \mathrm{H}) 6.85-6.70(\mathrm{~m}, 4 \mathrm{H}) 7.19-7.24(\mathrm{~m}, 1 \mathrm{H}) 7.28(\mathrm{t}$, $J=7.45 \mathrm{~Hz}, 2 \mathrm{H}) 7.36-7.38(\mathrm{~m}, 2 \mathrm{H}) 7.66-7.68(\mathrm{~m}, 2 \mathrm{H}) 7.78(\mathrm{~s}, 1 \mathrm{H}) ;{ }^{13} \mathbf{C} \mathbf{N M R}(100 \mathrm{MHz}$, $\left.\mathrm{CDCl}_{3}\right) \delta 55.6,106.6,110.7,115.3,123.6,125.2,126.4,127.6,127.7,128.6,129.3,131.7,137.3$, 139.5, 140.7, 159.5; HRESI-MS ( $m z)$ : Calculated for $\mathrm{C}_{18} \mathrm{H}_{16} \mathrm{~N}_{2} \mathrm{O}(\mathrm{M}+\mathrm{Na})$ : 299.1160 , found (M + Na): 299.1157.

(E)-1-(4-Methoxy-2-styrylphenyl)-1H-pyrazole (3ha). White solid; Yield - (42.5 mg, 77\%); $m p$ : $73-75{ }^{\circ} \mathrm{C}$; $\mathrm{R}_{\mathrm{f}}(10 \%$ EtOAc/Petroleum Ether) 0.3 ; Prepared as shown in general experimental procedure (a). IR $\left(\mathrm{KBr}, \mathrm{cm}^{-1}\right): 3055,3025,2957,2928,2841,2361,2336,1604,1576,1513$, 1288, 1220, 1044, 963; ${ }^{1} \mathbf{H}$ NMR $\left(400 \mathrm{MHz}, \mathrm{CDCl}_{3}\right) \delta 3.90(\mathrm{~s}, 3 \mathrm{H}) 6.44(\mathrm{t}, J=2.02 \mathrm{~Hz}, 1 \mathrm{H})$ $6.82(\mathrm{~d}, J=16.28 \mathrm{~Hz}, 1 \mathrm{H}) 6.90(\mathrm{dd}, J=8.59,2.78 \mathrm{~Hz}, 1 \mathrm{H}) 7.01(\mathrm{~d}, J=16.28 \mathrm{~Hz}, 1 \mathrm{H}) 7.22-$ $7.25(\mathrm{~m}, 2 \mathrm{H}) 7.29-7.38(\mathrm{~m}, 5 \mathrm{H}) 7.58(\mathrm{~d}, J=2.27 \mathrm{~Hz}, 1 \mathrm{H}) 7.75(\mathrm{~d}, J=1.52 \mathrm{~Hz}, 1 \mathrm{H}) ;{ }^{13} \mathbf{C}$ NMR $\left(100 \mathrm{MHz}, \mathrm{CDCl}_{3}\right) \delta 55.6,106.3,110.8,113.7,123.6,126.7,127.8,128.0,128.6,131.4$, 131.7, 132.4, 134.6, 136.8, 140.4, 159.4; HRESI-MS (mz): Calculated for $\mathrm{C}_{18} \mathrm{H}_{16} \mathrm{~N}_{2} \mathrm{O}(\mathrm{M}+\mathrm{Na})$ : 299.1160, found $(\mathrm{M}+\mathrm{Na}): 299.1163$. 
(E)-4-(1H-Pyrazol-1-yl)-3-styrylbenzonitrile (3ia). Off-white solid; Yield - (41.0 mg, 76\%); $\boldsymbol{m p}$ : $129-131{ }^{\circ} \mathrm{C} ; \mathrm{R}_{\mathrm{f}}$ (20\% EtOAc/Petroleum Ether) 0.40; Prepared as shown in general experimental procedure (a). IR $\left(\mathrm{KBr}, \mathrm{cm}^{-1}\right): 3059,3025,2923,2852,2230,1601,1518,1494$, 1394, 1334; ${ }^{1} \mathbf{H}$ NMR $\left(400 \mathrm{MHz}, \mathrm{CDCl}_{3}\right) \delta 6.53(\mathrm{t}, J=2.15 \mathrm{~Hz}, 1 \mathrm{H}) 7.02(\mathrm{~d}, J=16.32 \mathrm{~Hz}, 1 \mathrm{H})$ $7.12(\mathrm{~d}, J=16.32 \mathrm{~Hz}, 1 \mathrm{H}) 7.26-7.38(\mathrm{~m}, 3 \mathrm{H}) 7.43-7.44(\mathrm{~m}, 2 \mathrm{H}) 7.60-7.66(\mathrm{~m}, 2 \mathrm{H}) 7.73(\mathrm{~d}$, $J=2.27 \mathrm{~Hz}, 1 \mathrm{H}) 7.82(\mathrm{~d}, J=1.26 \mathrm{~Hz}, 1 \mathrm{H}) 8.03-8.04(\mathrm{~m}, 1 \mathrm{H}) ;{ }^{13} \mathbf{C ~ N M R}\left(100 \mathrm{MHz}, \mathrm{CDCl}_{3}\right) \delta$ 107.8, 111.9, 118.1, 122.3, 126.6, 126.9, 128.7, 128.8, 131.2, 131.4, 133.4, 133.5, 136.1, 141.5, 141.9; HRESI-MS (mz): Calculated for $\mathrm{C}_{18} \mathrm{H}_{13} \mathrm{~N}_{3}(\mathrm{M}+\mathrm{Na})$ : 294.1007, found $(\mathrm{M}+\mathrm{Na})$ : 294.1008.

(E)-1-(4-(1H-Pyrazol-1-yl)-3-styrylphenyl)ethan-1-one (3ja). Colorless Oil; Yield - (36.3 mg, 63\%); $\mathrm{R}_{\mathrm{f}}(30 \%$ EtOAc/Petroleum Ether) 0.4; Prepared as shown in general experimental procedure (a). IR $\left(\mathrm{KBr}, \mathrm{cm}^{-1}\right): 3057,3022,2923,2853,1684,1599,1495,1395,1283$; ${ }^{1} \mathbf{H}$ NMR $\left(400 \mathrm{MHz}, \mathrm{CDCl}_{3}\right) \delta 2.69(\mathrm{~s}, 3 \mathrm{H}) 6.51(\mathrm{t}, J=1.84 \mathrm{~Hz}, 1 \mathrm{H}) 7.07(\mathrm{~d}, J=16.28 \mathrm{~Hz}, 1 \mathrm{H}) 7.18(\mathrm{~d}$, $J=16.28 \mathrm{~Hz}, 1 \mathrm{H}) 7.29(\mathrm{~d}, J=7.07 \mathrm{~Hz}, 1 \mathrm{H}) 7.35(\mathrm{t}, J=7.45 \mathrm{~Hz}, 2 \mathrm{H}) 7.44(\mathrm{~d}, J=7.33 \mathrm{~Hz}, 2 \mathrm{H})$ $7.59(\mathrm{~d}, J=8.08 \mathrm{~Hz}, 1 \mathrm{H}) 7.74(\mathrm{~d}, J=2.27 \mathrm{~Hz}, 1 \mathrm{H}) 7.81-7.82(\mathrm{~m}, 1 \mathrm{H}) 7.94(\mathrm{dd}, J=8.34,1.77$ $\mathrm{Hz}, 1 \mathrm{H}) 8.35$ - $8.36(\mathrm{~m}, 1 \mathrm{H}) ;{ }^{13} \mathbf{C}$ NMR $\left(100 \mathrm{MHz}, \mathrm{CDCl}_{3}\right) \delta 26.8,107.3,123.4,126.0,126.8$, 127.2, 128.0, 128.3, 128.7, 131.4, 132.4, 132.5, 136.3, 136.6, 141.5, 141.9, 197.2; HRESI-MS (mz): Calculated for $\mathrm{C}_{19} \mathrm{H}_{16} \mathrm{~N}_{2} \mathrm{O}(\mathrm{M}+\mathrm{Na}): 311.1160$, found $(\mathrm{M}+\mathrm{Na}): 311.1162$.

(E)-1-(4-Nitro-2-styrylphenyl)-1H-pyrazole (3ka). Yellow solid; Yield - (20.4 mg, 35\%); $\boldsymbol{m p}$ : $141-143{ }^{\circ} \mathrm{C} ; \mathrm{R}_{\mathrm{f}}(20 \%$ EtOAc/Petroleum Ether) 0.50; Prepared as shown in general experimental procedure (a). IR $\left(\mathrm{KBr}, \mathrm{cm}^{-1}\right): 3084,3028,2923,2855,2362,2337,1610,1576,1523,1319$, 1344, 1338; ${ }^{1} \mathbf{H}$ NMR $\left(400 \mathrm{MHz}, \mathrm{CDCl}_{3}\right) \delta 6.55(\mathrm{t}, J=2.02 \mathrm{~Hz}, 1 \mathrm{H}) 7.09(\mathrm{~d}, J=16.24 \mathrm{~Hz}, 1 \mathrm{H})$ 7.20 - $7.26(\mathrm{~m}, 1 \mathrm{H}) 7.29$ - $7.39(\mathrm{~m}, 3 \mathrm{H}) 7.45-7.47(\mathrm{~m}, 2 \mathrm{H}) 7.67(\mathrm{~d}, J=8.59 \mathrm{~Hz}, 1 \mathrm{H}) 7.77(\mathrm{~d}$, $J=2.53 \mathrm{~Hz}, 1 \mathrm{H}) 7.84(\mathrm{~d}, J=1.52 \mathrm{~Hz}, 1 \mathrm{H}) 8.20(\mathrm{dd}, J=8.84,2.53 \mathrm{~Hz}, 1 \mathrm{H}) 8.62(\mathrm{~d}, J=2.53$ $\mathrm{Hz}, 1 \mathrm{H}) ;{ }^{13} \mathbf{C}$ NMR $\left(100 \mathrm{MHz}, \mathrm{CDCl}_{3}\right) \delta 107.9,122.3,122.4,122.7,126.6,127.0,128.8,131.5$, 133.3, 133.9, 136.0, 142.1, 142.8, 146.9; HRESI-MS (mz): Calculated for $\mathrm{C}_{17} \mathrm{H}_{13} \mathrm{~N}_{3} \mathrm{O}_{2}(\mathrm{M}+$ $\mathrm{Na})$ : 314.0905, found $(\mathrm{M}+\mathrm{Na}): 314.0903$.

(E)-2-(2-Styrylphenyl)pyridine (3la). Yellow Oil; Yield - (19.1 mg, 37\%); $\mathrm{R}_{\mathrm{f}}(10 \%$ EtOAc/petroleum ether) 0.3; Prepared as shown in general experimental procedure (b). IR (Neat, $\left.\mathrm{cm}^{-1}\right): 2362,2335,1647 ;{ }^{1} \mathbf{H}$ NMR $\left(400 \mathrm{MHz}, \mathrm{CDCl}_{3}\right) \delta 7.05(\mathrm{~d}, J=16.2 \mathrm{~Hz}, 1 \mathrm{H}), 7.20-7.32$ (m, $5 \mathrm{H}), 7.36$ - $7.46(\mathrm{~m}, 5 \mathrm{H}), 7.54-7.56(\mathrm{~m}, 1 \mathrm{H}), 7.71-7.77$ (m, $2 \mathrm{H}), 8.75(\mathrm{~d}, J=4.58 \mathrm{~Hz}, 1$ $\mathrm{H}) ;{ }^{13} \mathbf{C}$ NMR $\left(100 \mathrm{MHz}, \mathrm{CDCl}_{3}\right) \delta 121.8,125.0,126.2,126.6,127.5,128.6,128.7,130.1,130.2$, 135.7, 136.0, 137.5, 139.5, 149.5, 158.8; HRESI-MS (mz): Calculated for $\mathrm{C}_{19} \mathrm{H}_{15} \mathrm{~N}(\mathrm{M}+\mathrm{H})$ : 258.1283, found $(\mathrm{M}+\mathrm{H}): 258.1281$. 
(E)-1-(2-(4-Methylstyryl)phenyl)-1H-pyrazole (4aa). Light yellow oil; Yield - (35.0 mg, $67 \%) ; \mathrm{R}_{\mathrm{f}}(10 \%$ EtOAc/Petroleum Ether) 0.40; Prepared as shown in general experimental procedure (a). IR (KBr, cm $\left.{ }^{-1}\right): 3025,2920,2852,1597,1514,1488,1392 ;{ }^{1} \mathbf{H}$ NMR (400 MHz, $\left.\mathrm{CDCl}_{3}\right) \delta 2.33(\mathrm{~s}, 3 \mathrm{H}) 6.46(\mathrm{~s}, 1 \mathrm{H}) 6.88(\mathrm{~d}, J=16.28 \mathrm{~Hz}, 1 \mathrm{H}) 7.02(\mathrm{~d}, J=16.28 \mathrm{~Hz}, 1 \mathrm{H}) 7.12$ $(\mathrm{d}, J=7.84 \mathrm{~Hz}, 2 \mathrm{H}) 7.29(\mathrm{~d}, J=7.92 \mathrm{~Hz}, 2 \mathrm{H}) 7.33-7.45(\mathrm{~m}, 3 \mathrm{H}) 7.64-7.65$ (m, $1 \mathrm{H}) 7.74$ $7.77(\mathrm{~m}, 2 \mathrm{H}) ;{ }^{13} \mathrm{C}$ NMR $\left(100 \mathrm{MHz}, \mathrm{CDCl}_{3}\right) \delta 21.2,106.5,122.8,126.3,126.4,126.6,127.9$, 128.3, 129.3, 131.2, 131.5, 133.1, 134.2, 137.9, 138.6, 140.7; HRESI-MS (mz): Calculated for $\mathrm{C}_{18} \mathrm{H}_{16} \mathrm{~N}_{2}(\mathrm{M}+\mathrm{Na}): 283.1211$, found $(\mathrm{M}+\mathrm{Na}): 283.1212$.

(E)-1-(2-(4-Methoxystyryl)phenyl)-1H-pyrazole (4ab). Light yellow semi-solid; Yield - (40.0 $\mathrm{mg}, 72 \%) ; \mathrm{R}_{\mathrm{f}}(10 \%$ EtOAc/Petroleum Ether) 0.30; Prepared as shown in general experimental procedure (a). IR $\left(\mathrm{KBr}, \mathrm{cm}^{-1}\right)$ : 3055, 2928, 2841, 2361, 2336, 1604, 1513, 1289, 1044; ${ }^{1} \mathbf{H}$ NMR $\left(400 \mathrm{MHz}, \mathrm{CDCl}_{3}\right) \delta 3.80(\mathrm{~s}, 3 \mathrm{H}) 6.46(\mathrm{~s}, 1 \mathrm{H}) 6.79(\mathrm{~d}, J=16.24 \mathrm{~Hz}, 1 \mathrm{H}) 6.85(\mathrm{~d}, J=8.60 \mathrm{~Hz}$, $2 \mathrm{H}) 7.00(\mathrm{~d}, J=16.24 \mathrm{~Hz}, 1 \mathrm{H}) 7.32-7.35(\mathrm{~m}, 3 \mathrm{H}) 7.38-7.44(\mathrm{~m}, 2 \mathrm{H}) 7.65$ (s, $1 \mathrm{H}) 7.74$ $7.77(\mathrm{~m}, 2 \mathrm{H}) ;{ }^{13} \mathrm{C}$ NMR $\left(100 \mathrm{MHz}, \mathrm{CDCl}_{3}\right) \delta 55.3,106.5,114.1,121.6,126.3,126.4,127.7$, 127.9, 128.4, 129.8, 130.7, 131.5, 133.3, 128.5, 140.6, 159.5; HRESI-MS (mz): Calculated for $\mathrm{C}_{18} \mathrm{H}_{16} \mathrm{~N}_{2} \mathrm{O}(\mathrm{M}+\mathrm{Na}): 299.1160$, found $(\mathrm{M}+\mathrm{Na}): 299.1161$.

(E)-1-(2-(4-Fluorostyryl)phenyl)-1H-pyrazole (4ac). Light yellow oil; Yield - (46.0 mg, 87\%); $\mathrm{R}_{\mathrm{f}}(10 \%$ EtOAc/Petroleum Ether) 0.50; Prepared as shown in general experimental procedure (a). IR $\left(\mathrm{KBr}, \mathrm{cm}^{-1}\right): 3064,3041,2925,2388,2285,1598,1510,1393,1230 ;{ }^{1} \mathbf{H}$ NMR (400 MHz, $\left.\mathrm{CDCl}_{3}\right) \delta 6.47-6.48(\mathrm{~m}, 1 \mathrm{H}) 6.85(\mathrm{~d}, J=16.24 \mathrm{~Hz}, 1 \mathrm{H}) 6.98-7.02(\mathrm{~m}, 3 \mathrm{H}) 7.34$ - $7.44(\mathrm{~m}, 5$ H) $7.65(\mathrm{~s}, 1 \mathrm{H}) 7.74-7.78(\mathrm{~m}, 2 \mathrm{H}) ;{ }^{13} \mathrm{C} \mathrm{NMR}\left(100 \mathrm{MHz}, \mathrm{CDCl}_{3}\right) \delta 106.6,115.6\left(J_{C-F}=21.5\right.$ $\mathrm{Hz}), 123.6\left(J_{C-F}=2.2 \mathrm{~Hz}\right), 126.4\left(J_{C-F}=9.3 \mathrm{~Hz}\right), 128.13,128.15,128.2,128.4,129.9,131.5$, 132.9, $133.2\left(J_{C-F}=3.4 \mathrm{~Hz}\right), 138.7,140.7,162.4\left(J_{C-F}=246.2 \mathrm{~Hz}\right)$; HRESI-MS $(m z)$ : Calculated for $\mathrm{C}_{17} \mathrm{H}_{13} \mathrm{~N}_{2} \mathrm{~F}(\mathrm{M}+\mathrm{Na}): 287.0960$, found $(\mathrm{M}+\mathrm{Na}): 287.0957$.

(E)-1-(2-(4-Bromostyryl)phenyl)-1H-pyrazole (4ad). Off-white solid; Yield - (54.0 mg, 83\%); mp: $83-85^{\circ} \mathrm{C} ; \mathrm{R}_{\mathrm{f}}(20 \%$ EtOAc/Petroleum Ether) 0.5; Prepared as shown in general experimental procedure (a). IR $\left(\mathrm{KBr}, \mathrm{cm}^{-1}\right): 3138,3052,2921,2851,1514,1490,1393,1327 ;{ }^{1} \mathbf{H}$ NMR (400 $\left.\mathrm{MHz} \mathrm{CDCl}_{3}\right) \delta 6.48(\mathrm{t}, J=2.02 \mathrm{~Hz}, 1 \mathrm{H}) 6.95(\mathrm{~d}, J=4.29 \mathrm{~Hz}, 2 \mathrm{H}) 7.23-7.25(\mathrm{~m}, 2 \mathrm{H}) 7.37$ $7.44(\mathrm{~m}, 5 \mathrm{H}) 7.65(\mathrm{~d}, J=2.27 \mathrm{~Hz}, 1 \mathrm{H}) 7.74-7.78(\mathrm{~m}, 2 \mathrm{H}) ;{ }^{13} \mathbf{C} \mathbf{N M R}\left(100 \mathrm{MHz}, \mathrm{CDCl}_{3}\right) \delta$ 106.7, 121.7, 124.6, 126.3, 126.5, 128.1, 128.4, 128.5, 129.8, 131.46, 131.74, 132.66, 135.93, 138.73, 140.83; HRESI-MS (mz): Calculated for $\mathrm{C}_{17} \mathrm{H}_{13} \mathrm{~N}_{2} \mathrm{Br}(\mathrm{M}+\mathrm{Na}): 347.0160$, found $(\mathrm{M}+$ $\mathrm{Na}): 347.0161$.

(E)-1-(2-(3-Chlorostyryl)phenyl)-1H-pyrazole (4ae). Light yellow oil; Yield - (47.0 mg, 84\%); $\mathrm{R}_{\mathrm{f}}$ (10\% EtOAc/Petroleum Ether) 0.4; Prepared as shown in general experimental procedure (a). IR $\left(\mathrm{KBr}, \mathrm{cm}^{-1}\right): 3298,2924,2852,2342,2285,15921515,1489,1392 ;{ }^{1} \mathbf{H}$ NMR (400 MHz, $\left.\mathrm{CDCl}_{3}\right) \delta 6.49(\mathrm{~s}, 1 \mathrm{H}) 6.96(\mathrm{~s}, 2 \mathrm{H}) 7.22-7.25(\mathrm{~m}, 3 \mathrm{H}) 7.35-7.46(\mathrm{~m}, 5 \mathrm{H}) 7.65-7.66(\mathrm{~m}, 1$ H) 7.73 - $7.79(\mathrm{~m}, 2 \mathrm{H}) ;{ }^{13} \mathbf{C} \mathbf{N M R}\left(100 \mathrm{MHz}, \mathrm{CDCl}_{3}\right) \delta 106.7,124.7,125.4,126.3,126.6,126.7$, 
127.8, 128.4, 128.5, 129.7, 129.8, 131.4, 132.5, 134.6, 138.8, 138.9, 140.9; HRESI-MS (mz): Calculated for $\mathrm{C}_{17} \mathrm{H}_{13} \mathrm{~N}_{2} \mathrm{Cl}(\mathrm{M}+\mathrm{Na}): 303.0665$, found $(\mathrm{M}+\mathrm{Na})$ : 303.0667 .

(E)-1-(2-(2-Methylstyryl)phenyl)-1H-pyrazole (4af). Light yellow oil; Yield - (28.0 mg, 54\%); $\mathrm{R}_{\mathrm{f}}(20 \%$ EtOAc/Petroleum Ether) 0.60; Prepared as shown in general experimental procedure (a). IR $\left(\mathrm{KBr}, \mathrm{cm}^{-1}\right): 3052,3030,2956,2923,2853,2361,1511,1470,1390 ;{ }^{1} \mathbf{H}$ NMR $(400 \mathrm{MHz}$, $\left.\mathrm{CDCl}_{3}\right) \delta 2.06(\mathrm{~s}, 3 \mathrm{H}) 6.43(\mathrm{~d}, J=16.48 \mathrm{~Hz}, 1 \mathrm{H}) 6.49(\mathrm{t}, J=1.98 \mathrm{~Hz}, 1 \mathrm{H}) 6.97(\mathrm{~d}, J=16.48$ $\mathrm{Hz}, 1 \mathrm{H}) 7.21-7.23(\mathrm{~m}, 2 \mathrm{H}) 7.28-7.31(\mathrm{~m}, 4 \mathrm{H}) 7.36(\mathrm{t}, J=7.63 \mathrm{~Hz}, 1 \mathrm{H}) 7.50(\mathrm{~d}, J=2.14 \mathrm{~Hz}$, $1 \mathrm{H}) 7.62(\mathrm{~d}, J=7.93 \mathrm{~Hz}, 1 \mathrm{H}) 7.80(\mathrm{~d}, J=1.83 \mathrm{~Hz}, 1 \mathrm{H}) ;{ }^{13} \mathbf{C} \mathbf{N M R}\left(100 \mathrm{MHz}, \mathrm{CDCl}_{3}\right) \delta 19.9$, 106.6, 125.1, 125.5, 126.2, 126.4, 126.8, 127.8, 128.1, 128.4, 129.0, 130.4, 131.5, 133.3, 135.9, 136.0, 138.7, 140.7; HRESI-MS ( $m z)$ : Calculated for $\mathrm{C}_{18} \mathrm{H}_{16} \mathrm{~N}_{2}(\mathrm{M}+\mathrm{Na})$ : 283.1211, found (M + $\mathrm{Na)}: 283.1212$.

(E)-1-(2-(4-(tert-Butyl)styryl)phenyl)-1H-pyrazole (4ag). Yellow semi-solid; Yield - (41.0 mg, 68\%); $\mathrm{R}_{\mathrm{f}}(20 \%$ EtOAc/Petroleum Ether) 0.60; Prepared as shown in general experimental procedure (a). IR (KBr, cm $\left.{ }^{-1}\right): 3060,3033,2960,2925,2862,1514,1461,1392 ;{ }^{1} \mathbf{H}$ NMR (400 $\left.\mathrm{MHz}, \mathrm{CDCl}_{3}\right) \delta 1.31(\mathrm{~s}, 9 \mathrm{H}) 6.46(\mathrm{t}, J=2.02 \mathrm{~Hz}, 1 \mathrm{H}) 6.90(\mathrm{~d}, J=16.28 \mathrm{~Hz}, 1 \mathrm{H}) 7.03(\mathrm{~d}, J=$ $16.28 \mathrm{~Hz}, 1 \mathrm{H}) 7.32-7.37(\mathrm{~m}, 5 \mathrm{H}) 7.38-7.46(\mathrm{~m}, 2 \mathrm{H}) 7.65(\mathrm{~d}, J=2.27 \mathrm{~Hz}, 1 \mathrm{H}) 7.75-7.78$ $(\mathrm{m}, 2 \mathrm{H}) ;{ }^{13} \mathbf{C}$ NMR $\left(100 \mathrm{MHz}, \mathrm{CDCl}_{3}\right) \delta 31.2,34.6,106.5,123.1,125.6,126.3,126.4,126.5$, 127.9, 128.4, 131.1, 131.6, 133.2, 134.2, 138.6, 140.7, 151.2; HRESI-MS (mz): Calculated for $\mathrm{C}_{21} \mathrm{H}_{22} \mathrm{~N}_{2}(\mathrm{M}+\mathrm{Na}): 325.1681$, found $(\mathrm{M}+\mathrm{Na}): 325.1683$.

(E)-4-(2-(1H-Pyrazol-1-yl)styryl)benzonitrile (4ah). Light yellow solid; Yield - (49.5 mg, 91\%); $\boldsymbol{m p}$ : $97-99{ }^{\circ} \mathrm{C} ; \mathrm{R}_{\mathrm{f}}(20 \%$ EtOAc/Petroleum Ether) 0.30; Prepared as shown in general experimental procedure (a). IR $\left(\mathrm{KBr}, \mathrm{cm}^{-1}\right): 3118,3062,2955,2942,2224,1601,1512,1415$, 1393, 1330; ${ }^{1} \mathbf{H}$ NMR $\left(400 \mathrm{MHz}, \mathrm{CDCl}_{3}\right) \delta 6.50(\mathrm{t}, J=2.15 \mathrm{~Hz}, 1 \mathrm{H}) 7.02(\mathrm{~d}, J=16.32 \mathrm{~Hz}, 1 \mathrm{H})$ 7.09 (d, $J=16.32 \mathrm{~Hz}, 1 \mathrm{H}) 7.40-7.48(\mathrm{~m}, 5 \mathrm{H}) 7.59(\mathrm{~d}, J=8.34 \mathrm{~Hz}, 2 \mathrm{H}) 7.66(\mathrm{~d}, J=2.27 \mathrm{~Hz}$, $1 \mathrm{H}) 7.78-7.80(\mathrm{~m}, 2 \mathrm{H}) ;{ }^{13} \mathbf{C} \mathbf{~ N M R}\left(100 \mathrm{MHz}, \mathrm{CDCl}_{3}\right) \delta 106.9,110.8,118.9,126.3,126.7$, 127.0, 127.7, 128.5, 129.0, 129.0, 131.4, 132.1, 132.4, 139.0, 140.9, 141.5; HRESI-MS (mz): Calculated for $\mathrm{C}_{18} \mathrm{H}_{13} \mathrm{~N}_{3}(\mathrm{M}+\mathrm{Na}): 294.1007$, found $(\mathrm{M}+\mathrm{Na}): 294.1013$.

(E)-1-(2-(4-Nitrostyryl)phenyl)-1H-pyrazole (4ai). Yellow solid; Yield - (45.0 mg, 77\%); $\boldsymbol{m p}$ : $109-111{ }^{\circ} \mathrm{C} ; \mathrm{R}_{\mathrm{f}}$ (20\% EtOAc/Petroleum Ether) 0.30; Prepared as shown in general experimental procedure (a). IR $\left(\mathrm{KBr}, \mathrm{cm}^{-1}\right): 3069,2926,2852,1593,1514,1393,1340 ;{ }^{1} \mathbf{H} \mathbf{~ N M R}(400 \mathrm{MHz}$, $\left.\mathrm{CDCl}_{3}\right) \delta 6.52(\mathrm{t}, J=2.15 \mathrm{~Hz}, 1 \mathrm{H}) 7.08(\mathrm{~d}, J=16.32 \mathrm{~Hz}, 1 \mathrm{H}) 7.16(\mathrm{~d}, J=16.32 \mathrm{~Hz}, 1 \mathrm{H}) 7.44-$ $7.53(\mathrm{~m}, 5 \mathrm{H}) 7.68(\mathrm{~d}, J=2.27 \mathrm{~Hz}, 1 \mathrm{H}) 7.79-7.81(\mathrm{~m}, 2 \mathrm{H}) 8.17-8.20(\mathrm{~m}, 2 \mathrm{H}) ;{ }^{13} \mathbf{C} \mathbf{N M R}$ $\left(100 \mathrm{MHz}, \mathrm{CDCl}_{3}\right) \delta 106.9,124.1,126.3,126.8,127.1,128.5,128.6,128.6,129.2,131.4,132.0$, 139.1, 141.1, 143.5, 146.9; HRESI-MS $(m z)$ : Calculated for $\mathrm{C}_{17} \mathrm{H}_{13} \mathrm{~N}_{3} \mathrm{O}_{2}(\mathrm{M}+\mathrm{Na})$ : 314.0905 , found $(\mathrm{M}+\mathrm{Na})$ : 314.0907 . 
(E)-1-(4-(2-(1H-Pyrazol-1-yl)styryl)phenyl)ethan-1-one (4aj). Light yellow solid; Yield (34.5 mg, 60\%); $\boldsymbol{m p}$ : $121-123{ }^{\circ} \mathrm{C}$; $\mathrm{R}_{\mathrm{f}}(20 \%$ EtOAc/Petroleum Ether) 0.25; Prepared as shown in general experimental procedure (a). IR $\left(\mathrm{KBr}, \mathrm{cm}^{-1}\right): 3116,3061,3006,2923,2854,1678,1599$, 1515, 1394, 1267; ${ }^{1} \mathbf{H}$ NMR (400 MHz, CDCl $) \delta 2.58(\mathrm{~s}, 3 \mathrm{H}) 6.50(\mathrm{t}, J=2.02 \mathrm{~Hz}, 1 \mathrm{H}) 7.07$ (s, $2 \mathrm{H}) 7.38$ - $7.47(\mathrm{~m}, 5 \mathrm{H}) 7.66(\mathrm{~d}, J=2.53 \mathrm{~Hz}, 1 \mathrm{H}) 7.77$ - $7.80(\mathrm{~m}, 2 \mathrm{H}) 7.89$ - $7.91(\mathrm{~m}, 2 \mathrm{H})$; ${ }^{13} \mathrm{C}$ NMR $\left(100 \mathrm{MHz}, \mathrm{CDCl}_{3}\right) \delta$ 26.6, 106.8, 126.3, 126.7, 126.7, 128.5, 128.7, 128.8, 129.8, 131.4, 132.4, 136.1, 139.0, 140.9, 141.6, 197.4; HRESI-MS (mz): Calculated for $\mathrm{C}_{19} \mathrm{H}_{16} \mathrm{~N}_{2} \mathrm{O}(\mathrm{M}$ $+\mathrm{Na}): 311.1160$, found $(\mathrm{M}+\mathrm{Na}): 311.1158$.

Ethyl (E)-4-(2-(1H-yrazol-1-yl)styryl)benzoate (4ak). Brown solid; Yield - (60.2 mg, 92\%); mp: $75-77{ }^{\circ} \mathrm{C} ; \quad \mathrm{R}_{\mathrm{f}}(20 \%$ EtOAc/Petroleum Ether) 0.40; Prepared as shown in general experimental procedure (a). IR $\left(\mathrm{KBr}, \mathrm{cm}^{-1}\right): 3060,2980,2929,2361,1712,1604,1515,1393$, 1276; ${ }^{1} \mathbf{H}$ NMR $\left(400 \mathrm{MHz}, \mathrm{CDCl}_{3}\right) \delta 1.39(\mathrm{t}, J=7.07 \mathrm{~Hz}, 3 \mathrm{H}) 4.36(\mathrm{q}, J=7.24 \mathrm{~Hz}, 2 \mathrm{H}) 6.48$ $6.50(\mathrm{~m}, 1 \mathrm{H}) 7.06(\mathrm{~s}, 2 \mathrm{H}) 7.39-7.46(\mathrm{~m}, 5 \mathrm{H}) 7.66(\mathrm{~d}, J=2.27 \mathrm{~Hz}, 1 \mathrm{H}) 7.77$ - 7.79 (m, $2 \mathrm{H})$ $7.99(\mathrm{~d}, J=8.34 \mathrm{~Hz}, 2 \mathrm{H}) ;{ }^{13} \mathbf{C}$ NMR $\left(100 \mathrm{MHz}, \mathrm{CDCl}_{3}\right) \delta 14.3,60.9,106.8,126.3,126.4,126.5$, 126.6, 128.5, 129.5, 129.9, 130.0, 131.4, 132.5, 138.9, 140.9, 141.3, 166.3; HRESI-MS (mz): Calculated for $\mathrm{C}_{20} \mathrm{H}_{18} \mathrm{~N}_{2} \mathrm{O}_{2}(\mathrm{M}+\mathrm{Na}): 341.1266$, found $(\mathrm{M}+\mathrm{Na}): 341.1268$.

(E)-1-(2-(2-(Thiophen-2-yl)vinyl)phenyl)-1H-pyrazole (4al). Orange oil; Yield - (30.5 mg, $62 \%) ; \mathrm{R}_{\mathrm{f}}(10 \%$ EtOAc/Petroleum Ether) 0.40; Prepared as shown in general experimental procedure (a). IR (KBr, cm $\left.{ }^{-1}\right): 3104,3068,2921,2805,2382,2284,1623,1597,1515,1392$, 949; ${ }^{1} \mathbf{H}$ NMR $\left(400 \mathrm{MHz}, \mathrm{CDCl}_{3}\right) \delta 6.47-6.48(\mathrm{~m}, 1 \mathrm{H}) 6.75(\mathrm{~d}, J=16.08 \mathrm{~Hz}, 1 \mathrm{H}) 6.96-6.98$ $(\mathrm{m}, 1 \mathrm{H}) 7.03(\mathrm{~d}, J=3.28 \mathrm{~Hz}, 1 \mathrm{H}) 7.12-7.18(\mathrm{~m}, 2 \mathrm{H}) 7.33-7.44(\mathrm{~m}, 3 \mathrm{H}) 7.65(\mathrm{~d}, J=2.02 \mathrm{~Hz}$, $1 \mathrm{H}) 7.71(\mathrm{~d}, J=7.58 \mathrm{~Hz}, 1 \mathrm{H}) 7.78(\mathrm{~s}, 1 \mathrm{H}) ;{ }^{13} \mathrm{C}$ NMR $\left(100 \mathrm{MHz}, \mathrm{CDCl}_{3}\right) \delta$ 106.6, 123.4, 124.2, 124.9, 126.3, 126.4, 126.5, 127.6, 128.1, 131.5, 132.6, 138.5, 140.8, 142.5; HRESI-MS (mz): Calculated for $\mathrm{C}_{15} \mathrm{H}_{12} \mathrm{~N}_{2} \mathrm{~S}(\mathrm{M}+\mathrm{Na}): 257.0619$, found $(\mathrm{M}+\mathrm{Na}): 257.0616$.

(E)-1-(2-(Prop-1-en-1-yl)phenyl)-1H-pyrazole (4am). Colorless oil; Yield - (24.0 mg, 65\%); $\mathbf{R}_{\mathrm{f}}$ (20\% EtOAc/Petroleum Ether) 0.65; Prepared as shown in general experimental procedure (a). IR $\left(\mathrm{KBr}, \mathrm{cm}^{-1}\right): 3111,3037,2963,2914,2852,2437,2361,2336,1692,1652,1599,1515,1492$, 1393, 1328; ${ }^{1} \mathbf{H}$ NMR (400 MHz, $\left.\mathrm{CDCl}_{3}\right) \delta 1.82$ - $1.83(\mathrm{~m}, 3 \mathrm{H}) 6.19$ - 6.22 (m, $\left.2 \mathrm{H}\right) 6.43$ - 6.45 $(\mathrm{m}, 1 \mathrm{H}) 7.26-7.37(\mathrm{~m}, 3 \mathrm{H}) 7.57-7.62(\mathrm{~m}, 2 \mathrm{H}) 7.73(\mathrm{~d}, J=1.77 \mathrm{~Hz}, 1 \mathrm{H}) ;{ }^{13} \mathbf{C}$ NMR $(100$ $\left.\mathrm{MHz} \mathrm{CDCl}_{3}\right) \delta 18.8,106.2,126.1,126.3,126.5,127.3,128.3,128.7,131.3,133.5,137.9,140.4$; HRESI-MS $(m z)$ : Calculated for $\mathrm{C}_{12} \mathrm{H}_{12} \mathrm{~N}_{2}(\mathrm{M}+\mathrm{Na}): 207.0898$, found $(\mathrm{M}+\mathrm{Na}): 207.0895$.

(E)-1-(2-(Pent-1-en-1-yl)phenyl)-1H-pyrazolepyrazole (4an). Yellow oil; Yield - (25.0 mg, 29\%); $\mathrm{R}_{\mathrm{f}}(20 \%$ EtOAc/Petroleum Ether) 0.80; Prepared as shown in general experimental procedure (a). IR $\left(\mathrm{KBr}, \mathrm{cm}^{-1}\right): 3107,3068,3039,2959,2867,2334,1694,1648,1600,1515$, 1491, 1491, 1459, 1393; ${ }^{1} \mathbf{H}$ NMR (400 MHz, $\left.\mathrm{CDCl}_{3}\right) \delta 0.91(\mathrm{t}, J=7.36 \mathrm{~Hz}, 3 \mathrm{H}) 1.41-1.47(\mathrm{~m}$, $2 \mathrm{H}) 2.12(\mathrm{dtd}, J=7.29,5.01,2.40 \mathrm{~Hz}, 2 \mathrm{H}) 6.17-6.19(\mathrm{~m}, 2 \mathrm{H}) 6.43-6.44(\mathrm{~m}, 1 \mathrm{H}) 7.26-7.39$ $(\mathrm{m}, 3 \mathrm{H}) 7.58-7.62(\mathrm{~m}, 2 \mathrm{H}) 7.73(\mathrm{~s}, 1 \mathrm{H}) ;{ }^{13} \mathbf{C ~ N M R}\left(100 \mathrm{MHz}, \mathrm{CDCl}_{3}\right) \delta$ 13.7, 22.3, 35.2, 
106.2, 125.1, 126.26, 126.6, 127.3, 128.2, 131.3, 133.5, 133.9, 138.0, 140.4; HRESI-MS (mz): Calculated for $\mathrm{C}_{14} \mathrm{H}_{16} \mathrm{~N}_{2}(\mathrm{M}+\mathrm{Na})$ : 235.1211, found $(\mathrm{M}+\mathrm{Na})$ : 235.1214.

(1-(2,6-di((E)-Pent-1-en-1-yl)phenyl)-1H-pyrazole (4an'). Light yellow oil; Yield - (16.0 mg, $14 \%) ; \mathrm{R}_{\mathrm{f}}(20 \% \mathrm{EtOAc/Petroleum} \mathrm{Ether)} \mathrm{0.70;} \mathrm{Prepared} \mathrm{as} \mathrm{shown} \mathrm{in} \mathrm{general} \mathrm{experimental}$ procedure (a). IR $\left(\mathrm{KBr}, \mathrm{cm}^{-1}\right): 3061,2959,2927,2867,2362,1647,1583,1513,1461,1390$, 966; ${ }^{1} \mathrm{H}$ NMR $\left(400 \mathrm{MHz}, \mathrm{CDCl}_{3}\right) \delta 0.86(\mathrm{t}, J=7.33 \mathrm{~Hz}, 6 \mathrm{H}) 1.37$ (sxt, $\left.J=7.33 \mathrm{~Hz}, 4 \mathrm{H}\right) 2.00$ $2.06(\mathrm{~m}, 4 \mathrm{H}) 5.72(\mathrm{~d}, J=15.84 \mathrm{~Hz}, 2 \mathrm{H}) 6.14(\mathrm{t}, J=6.95 \mathrm{~Hz}, 1 \mathrm{H}) 6.10(\mathrm{t}, J=6.95 \mathrm{~Hz}, 1 \mathrm{H})$ $6.45(\mathrm{t}, J=2.15 \mathrm{~Hz}, 1 \mathrm{H}) 7.33(\mathrm{t}, J=7.71 \mathrm{~Hz}, 1 \mathrm{H}) 7.45(\mathrm{~d}, J=8.08 \mathrm{~Hz}, 3 \mathrm{H}) 7.75(\mathrm{~d}, J=1.52$ $\mathrm{Hz}, 1 \mathrm{H}) ;{ }^{13} \mathbf{C}$ NMR $\left(100 \mathrm{MHz}, \mathrm{CDCl}_{3}\right) \delta 13.6,22.2,35.8,105.9,124.0,124.5,129.0,132.3$, 133.9, 135.3, 136.5, 140.1; HRESI-MS (mz): Calculated for $\mathrm{C}_{19} \mathrm{H}_{24} \mathrm{~N}_{2}(\mathrm{M}+\mathrm{Na})$ : 303.1837 , found $(\mathrm{M}+\mathrm{Na})$ : 303.1837 .

1-(2-Vinylphenyl)-1H-pyrazole (6aa). Light Yellow Oil; Yield - (26.0 mg, 51\%); $\mathrm{R}_{\mathrm{f}}(10 \%$ EtOAc/Petroleum Ether) 0.55; Prepared as shown in general experimental procedure (b). IR $\left(\mathrm{KBr}, \mathrm{cm}^{-1}\right): 2923,2852,2361,2361,2085,1840,1630,1605,1514,1489,1392 ;{ }^{1} \mathbf{H}$ NMR $(400$ $\left.\mathrm{MHz}, \mathrm{CDCl}_{3}\right) \delta 5.30(\mathrm{~d}, J=11.12 \mathrm{~Hz}, 1 \mathrm{H}) 5.73(\mathrm{~d}, J=17.48 \mathrm{~Hz}, 1 \mathrm{H}) 6.44-6.45(\mathrm{~m}, 1 \mathrm{H}) 6.57$ $(\mathrm{dd}, J=17.48,11.12 \mathrm{~Hz}, 1 \mathrm{H}) 7.36-7.42(\mathrm{~m}, 3 \mathrm{H}) 7.63-7.67(\mathrm{~m}, 2 \mathrm{H}) 7.74(\mathrm{~s}, 1 \mathrm{H}) ;{ }^{13} \mathbf{C}$ NMR $\left(100 \mathrm{MHz}, \mathrm{CDCl}_{3}\right) \delta 106.4,116.6,126.1,126.6,128.3,128.4,131.4,132.3,133.2,138.5,140.6$; HRESI-MS $(m z)$ : Calculated for $\mathrm{C}_{11} \mathrm{H}_{9} \mathrm{~N}_{2} \mathrm{Cl}(\mathrm{M}+\mathrm{Na})$ : 193.0742 , found $(\mathrm{M}+\mathrm{Na}): 193.0745$.

1-(2-Fluoro-6-vinylphenyl)-1H-pyrazole (6da). Brown Oil; Yield - (28.0 mg, 44\%); $\mathrm{R}_{\mathrm{f}}(10 \%$ EtOAc/Petroleum Ether) 0.35; Prepared as shown in general experimental procedure (b). IR $\left(\mathrm{KBr}, \mathrm{cm}^{-1}\right): 2920,2851,2362,2337,1610,1577,1520,1475,1389,1264 ;{ }^{1} \mathbf{H}$ NMR $(400 \mathrm{MHz}$, $\left.\mathrm{CDCl}_{3}\right) \delta 5.31(\mathrm{~d}, J=11.04 \mathrm{~Hz}, 1 \mathrm{H}) 5.73(\mathrm{~d}, J=17.52 \mathrm{~Hz}, 1 \mathrm{H}) 6.33(\mathrm{dd}, J=17.52,11.04 \mathrm{~Hz}, 1$ H) $6.49(\mathrm{t}, J=2.02 \mathrm{~Hz}, 1 \mathrm{H}) 7.11-7.16(\mathrm{~m}, 1 \mathrm{H}) 7.39(\mathrm{td}, J=8.02,5.43 \mathrm{~Hz}, 1 \mathrm{H}) 7.45-7.49(\mathrm{~m}$, $1 \mathrm{H}) 7.59(\mathrm{~s}, 1 \mathrm{H}) 7.79-7.80(\mathrm{~m}, 1 \mathrm{H}) ;{ }^{13} \mathbf{C} \mathbf{N M R}\left(100 \mathrm{MHz}, \mathrm{CDCl}_{3}\right) \delta 106.6,115.2\left(J_{C-F}=20.1\right.$ $\mathrm{Hz}), 117.9,121.3\left(J_{C-F}=3.5 \mathrm{~Hz}\right), 126.7\left(J_{C-F}=12.6 \mathrm{~Hz}\right), 129.8\left(J_{C-F}=8.7 \mathrm{~Hz}\right), 130.9\left(J_{C-F}=3.2\right.$ $\mathrm{Hz}), 132.4,137.6,141.0,157.7\left(J_{C-F}=249.5 \mathrm{~Hz}\right)$; HRESI-MS $(\mathrm{mz})$ : Calculated for $\mathrm{C}_{11} \mathrm{H}_{9} \mathrm{~N}_{2} \mathrm{~F}$ $(\mathrm{M}+\mathrm{Na}): 211.0647$, found $(\mathrm{M}+\mathrm{Na}): 211.0645$.

1-(4-Fluoro-2-vinylphenyl)-1H-pyrazole (6ea). Brown Oil; Yield - (31.0 mg, 48\%); $\mathrm{R}_{\mathrm{f}}(10 \%$ EtOAc/Petroleum Ether) 0.55; Prepared as shown in general experimental procedure (b). IR $\left(\mathrm{KBr}, \mathrm{cm}^{-1}\right): 2924,2854,2362,2140,1652,1615,1584,1516,1421,1330,1268,1023 ;{ }^{1} \mathbf{H}$ NMR $\left(400 \mathrm{MHz}, \mathrm{CDCl}_{3}\right) \delta 5.35(\mathrm{~d}, J=10.96 \mathrm{~Hz}, 1 \mathrm{H}) 5.74(\mathrm{~d}, J=17.48 \mathrm{~Hz}, 1 \mathrm{H}) 6.43-6.50(\mathrm{~m}, 2 \mathrm{H})$ $7.06(\mathrm{td}, J=8.21,2.84 \mathrm{~Hz}, 1 \mathrm{H}) 7.26-7.38(\mathrm{~m}, 2 \mathrm{H}) 7.58(\mathrm{~d}, J=2.24 \mathrm{~Hz}, 1 \mathrm{H}) 7.72-7.73(\mathrm{~m}, 1$ $\mathrm{H}) ;{ }^{13} \mathbf{C}$ NMR $\left(100 \mathrm{MHz}, \mathrm{CDCl}_{3}\right) \delta 106.6,112.8\left(J_{C-F}=23.3 \mathrm{~Hz}\right), 115.3\left(J_{C-F}=23.0 \mathrm{~Hz}\right), 117.8$, $128.2\left(J_{C-F}=9.0 \mathrm{~Hz}\right), 131.4\left(J_{C-F}=1.9 \mathrm{~Hz}\right), 131.5,134.7,135.6\left(J_{C-F}=8.3 \mathrm{~Hz}\right), 140.8,162.3\left(J_{C-}\right.$ $F=246.2 \mathrm{~Hz})$; HRESI-MS $(m z)$ : Calculated for $\mathrm{C}_{11} \mathrm{H}_{9} \mathrm{~N}_{2} \mathrm{Cl}(\mathrm{M}+)$ : 188.0750 , found $(\mathrm{M}+)$ : 188.0752 . 
1-(5-Chloro-2-vinylphenyl)-1H-pyrazole (6fa). Light Brown Oil; Yield - (34.5 mg, 56\%); $\mathrm{R}_{\mathrm{f}}$ (10\% EtOAc/Petroleum Ether) 0.60; Prepared as shown in general experimental procedure (b). IR $\left(\mathrm{KBr}, \mathrm{cm}^{-1}\right): 2958,2922,2851,2362,1724,1595,1516,1487,1401,1034 ;{ }^{1} \mathbf{H}$ NMR (400 $\left.\mathrm{MHz}, \mathrm{CDCl}_{3}\right) \delta 5.33(\mathrm{dd}, J=10.96,0.76 \mathrm{~Hz}, 1 \mathrm{H}) 5.72(\mathrm{dd}, J=17.52,0.76 \mathrm{~Hz}, 1 \mathrm{H}) 6.46(\mathrm{~s}, 1$ H) $6.55(\mathrm{dd}, J=17.52,10.96 \mathrm{~Hz}, 1 \mathrm{H}) 7.36(\mathrm{dd}, J=8.32,1.92 \mathrm{~Hz}, 1 \mathrm{H}) 7.45(\mathrm{~d}, J=2.12 \mathrm{~Hz}, 1$ H) $7.58(\mathrm{~d}, J=8.32 \mathrm{~Hz}, 1 \mathrm{H}) 7.64(\mathrm{~d}, J=1.88 \mathrm{~Hz}, 1 \mathrm{H}) 7.74-7.75(\mathrm{~m}, 1 \mathrm{H}) ;{ }^{13} \mathbf{C}$ NMR $(100$ $\left.\mathrm{MHz}, \mathrm{CDCl}_{3}\right) \delta 106.9,117.1,126.1,127.8,128.4,131.3,131.4,131.6,133.7,139.1,141.1$; HRESI-MS $(m z)$ : Calculated for $\mathrm{C}_{11} \mathrm{H}_{9} \mathrm{~N}_{2} \mathrm{Cl}(\mathrm{M}+\mathrm{Na}): 227.0352$, found $(\mathrm{M}+\mathrm{Na}): 227.0363$.

2-(1H-Pyrazol-1-yl)benzaldehyde (7). White Solid; Yield - (33.6 mg, 98\%); $\mathrm{R}_{\mathrm{f}}(10 \%$ EtOAc/Petroleum Ether) 0.40; Prepared as shown in general experimental procedure (d). IR $\left(\mathrm{KBr}, \mathrm{cm}^{-1}\right):$ 2954, 2924, 2853, 1690, 1600, 1519, 1493, 1458; ${ }^{1} \mathbf{H} \mathbf{~ N M R}\left(400 \mathrm{MHz}, \mathrm{CDCl}_{3}\right) \delta$ $6.55(\mathrm{~s}, 1 \mathrm{H}) 7.51(\mathrm{t}, J=7.80 \mathrm{~Hz}, 2 \mathrm{H}) 7.68(\mathrm{t}, J=7.72 \mathrm{~Hz}, 2 \mathrm{H}) 7.83(\mathrm{~d}, J=15.32 \mathrm{~Hz}, 2 \mathrm{H}) 8.02$ $(\mathrm{d}, J=7.83 \mathrm{~Hz}, 1 \mathrm{H}) 10.02(\mathrm{~s}, 1 \mathrm{H}) ;{ }^{13} \mathbf{C ~ N M R}\left(100 \mathrm{MHz}, \mathrm{CDCl}_{3}\right) \delta 108.0,124.4,128.0,128.7$, 130.2, 130.7, 134.2, 141.9, 142.2, 190.1; HRESI-MS (mz): Calculated for $\mathrm{C}_{11} \mathrm{H}_{9} \mathrm{~N}_{2} \mathrm{Cl}(\mathrm{M}+\mathrm{Na})$ : 195.0534, found $(\mathrm{M}+\mathrm{Na}): 195.0535$.

\section{References}

1. a) Z. Xu, H. Le, Z. Ren, W. Du, W. Du, J. Lang, Tetrahedron 2011, 67, 5282; b) P. M. Liu, C. G. Frost, Org. Lett. 2013, 15, 5826.

2. K. Park, T. Palani, A. Pyo, S. Lee, Tetrahedron Letters 2012, 53, 733.

3. X. Yang, X. Jin, C. Wang, Adv. Synth. Catal. 2016, 358, 2436.

4. S. Dillinger, P. Bertus, P. Pale, Org. Lett. 2001, 3, 1661.

5. B. Sun, T. Yoshino, S. Matsunaga, M. Kanai, Adv. Synth. Catal. 2014, 356, 1491. 


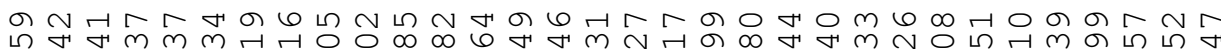
ம

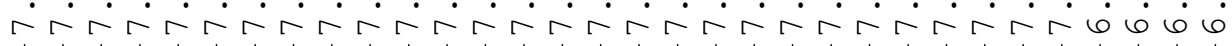
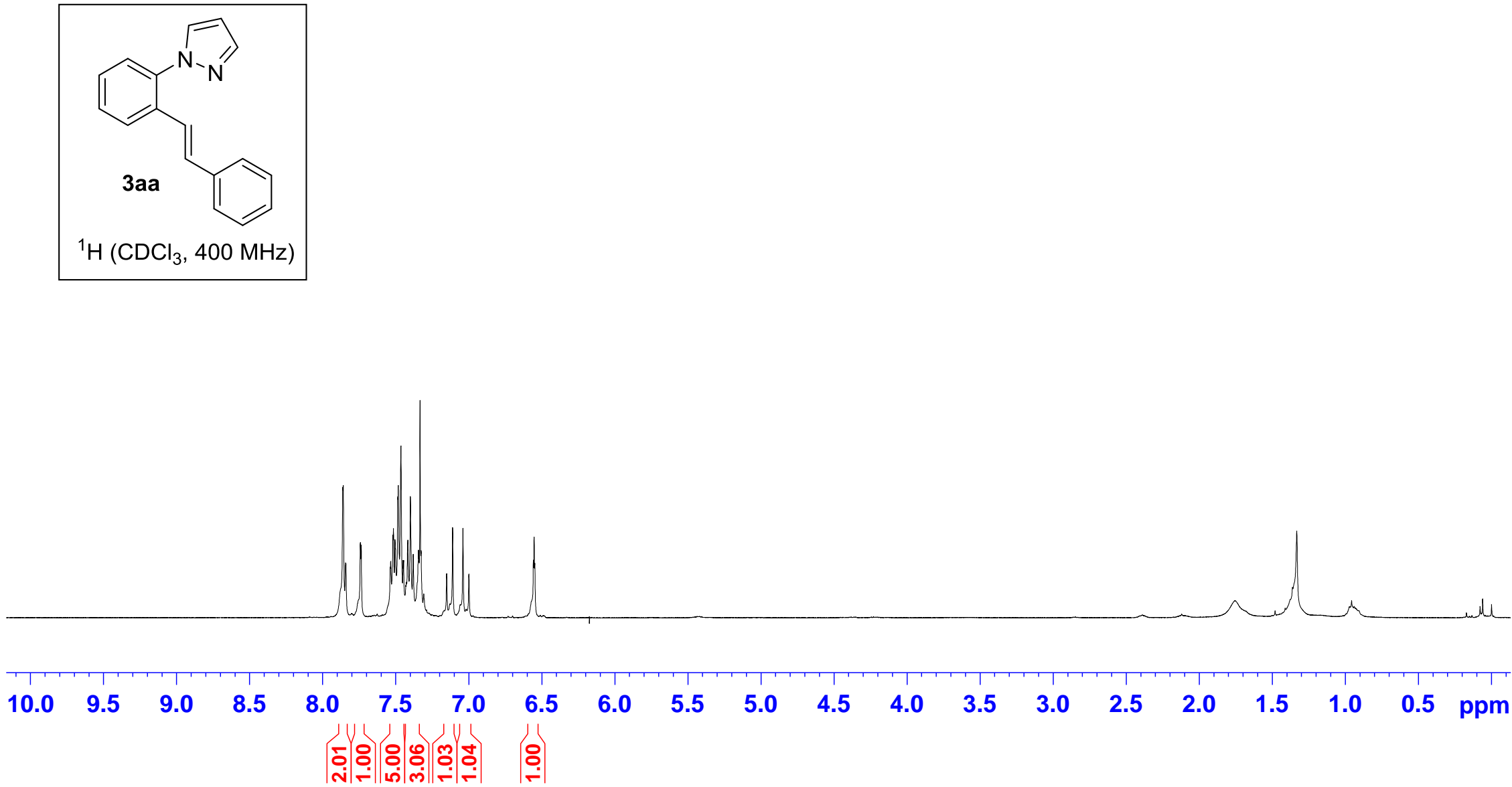

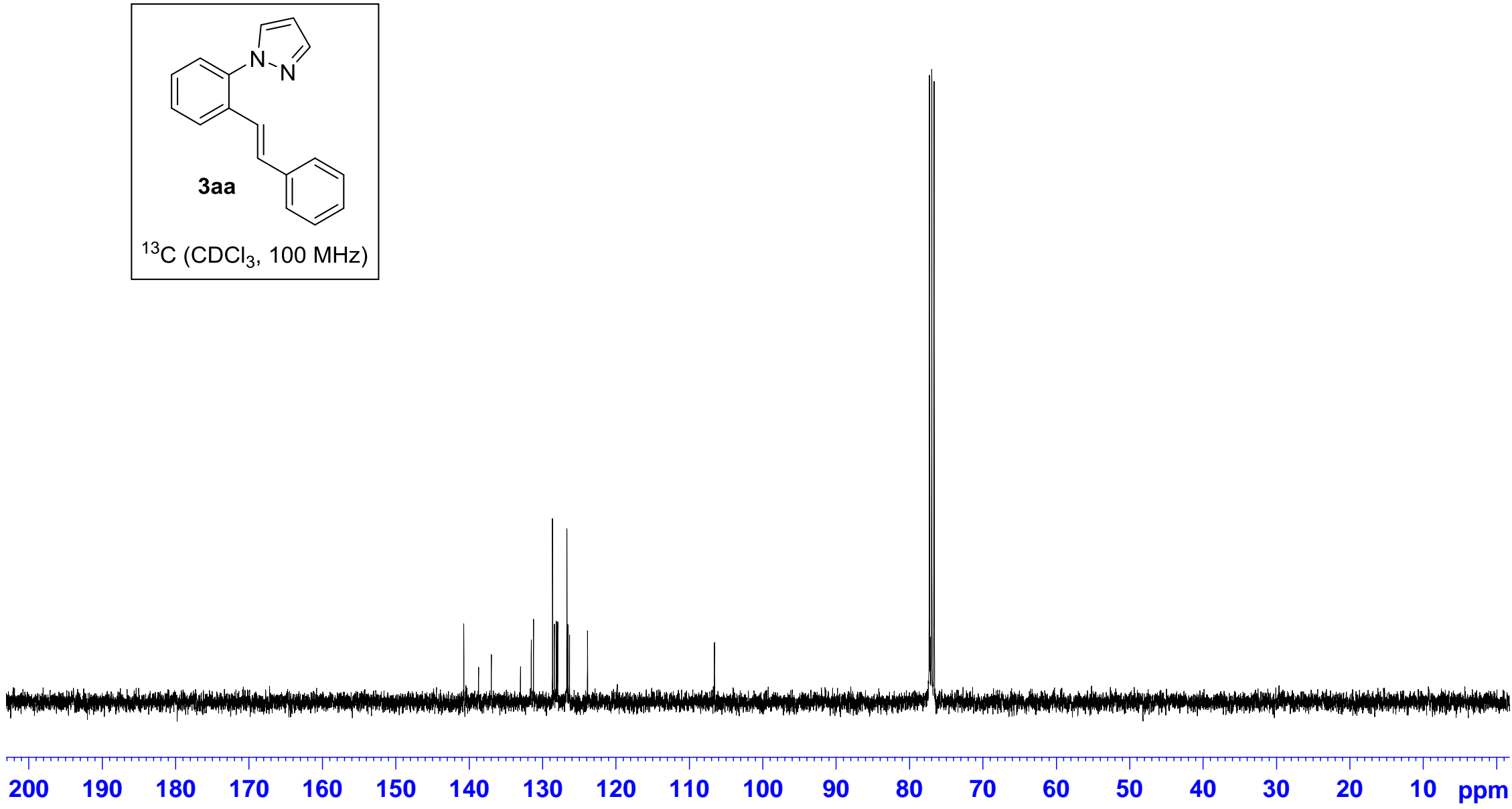


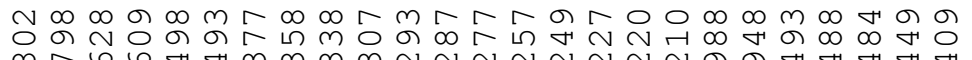

$\infty \pi \in G$ म

arrararararararara

L
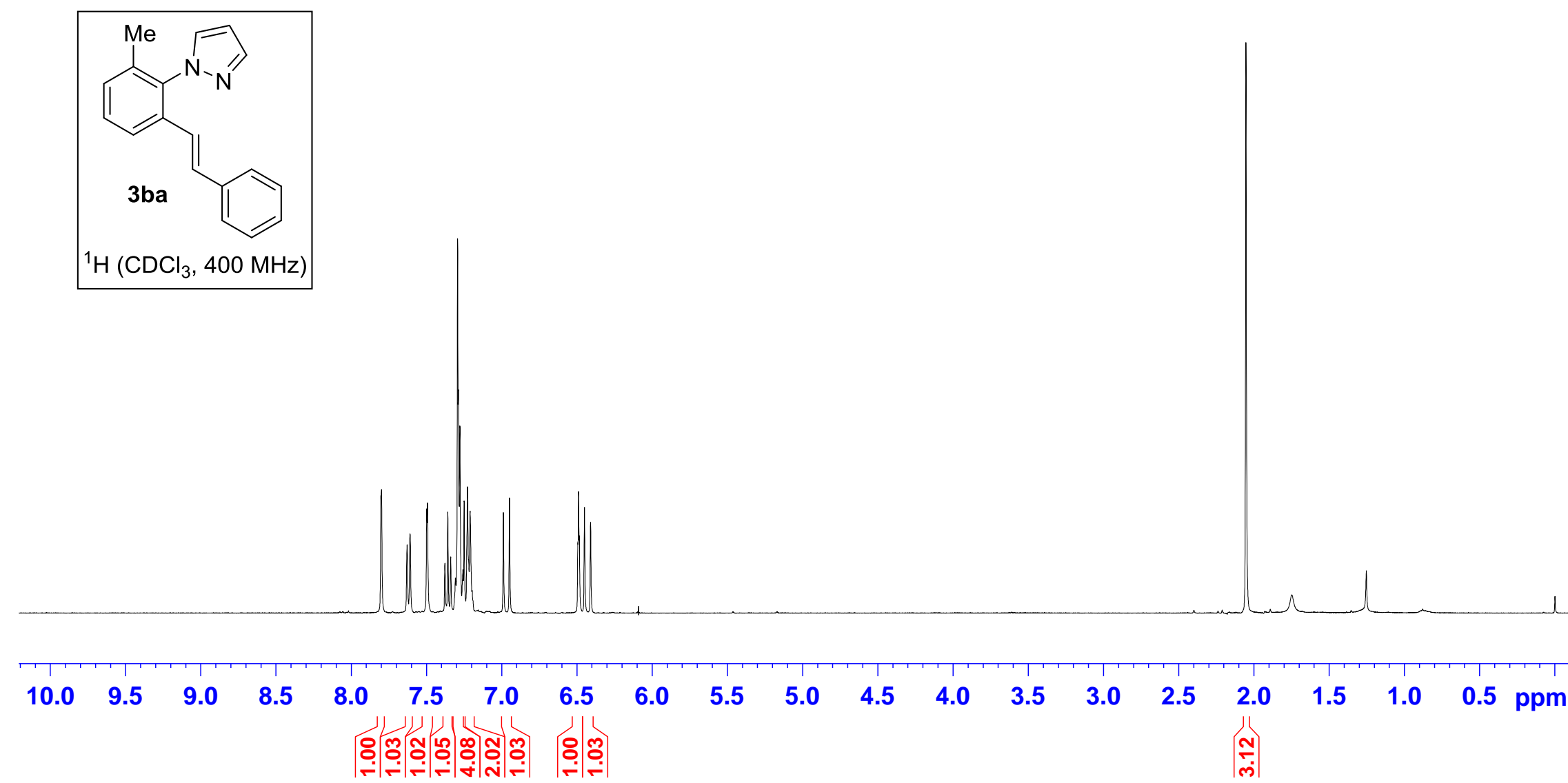


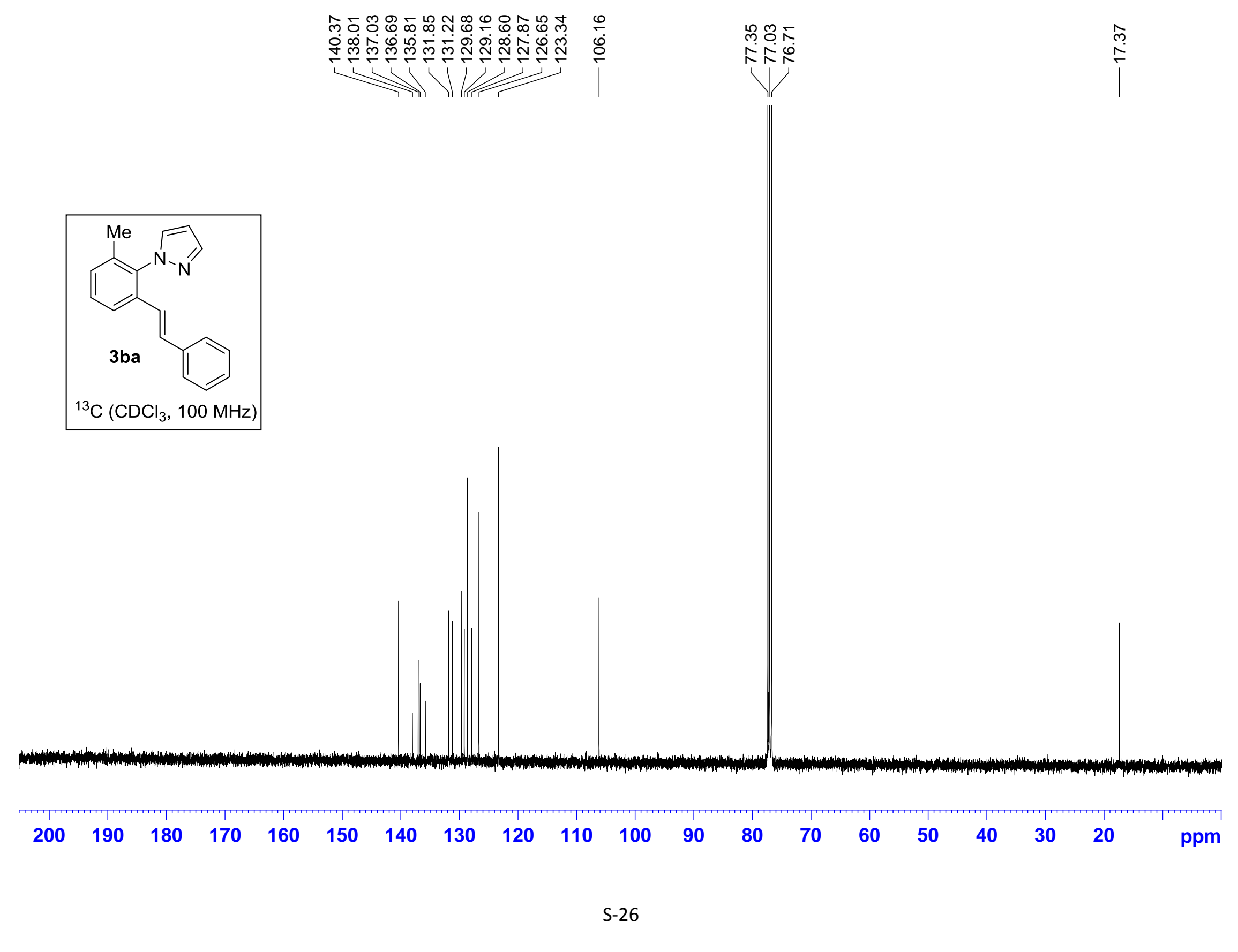


๒ூ

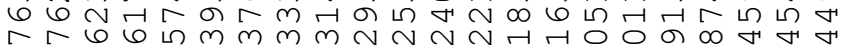
rarararararararar $\longrightarrow$

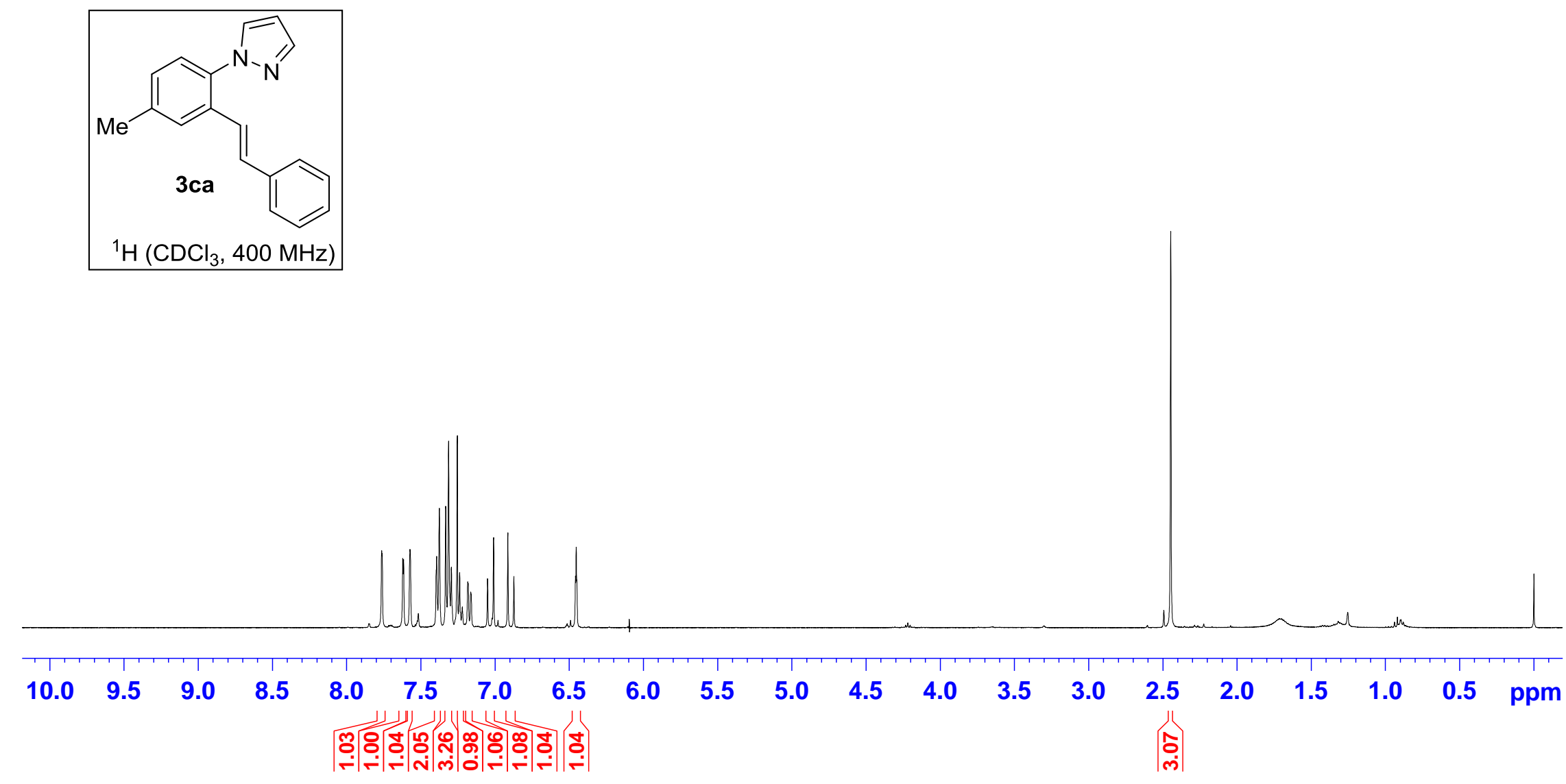



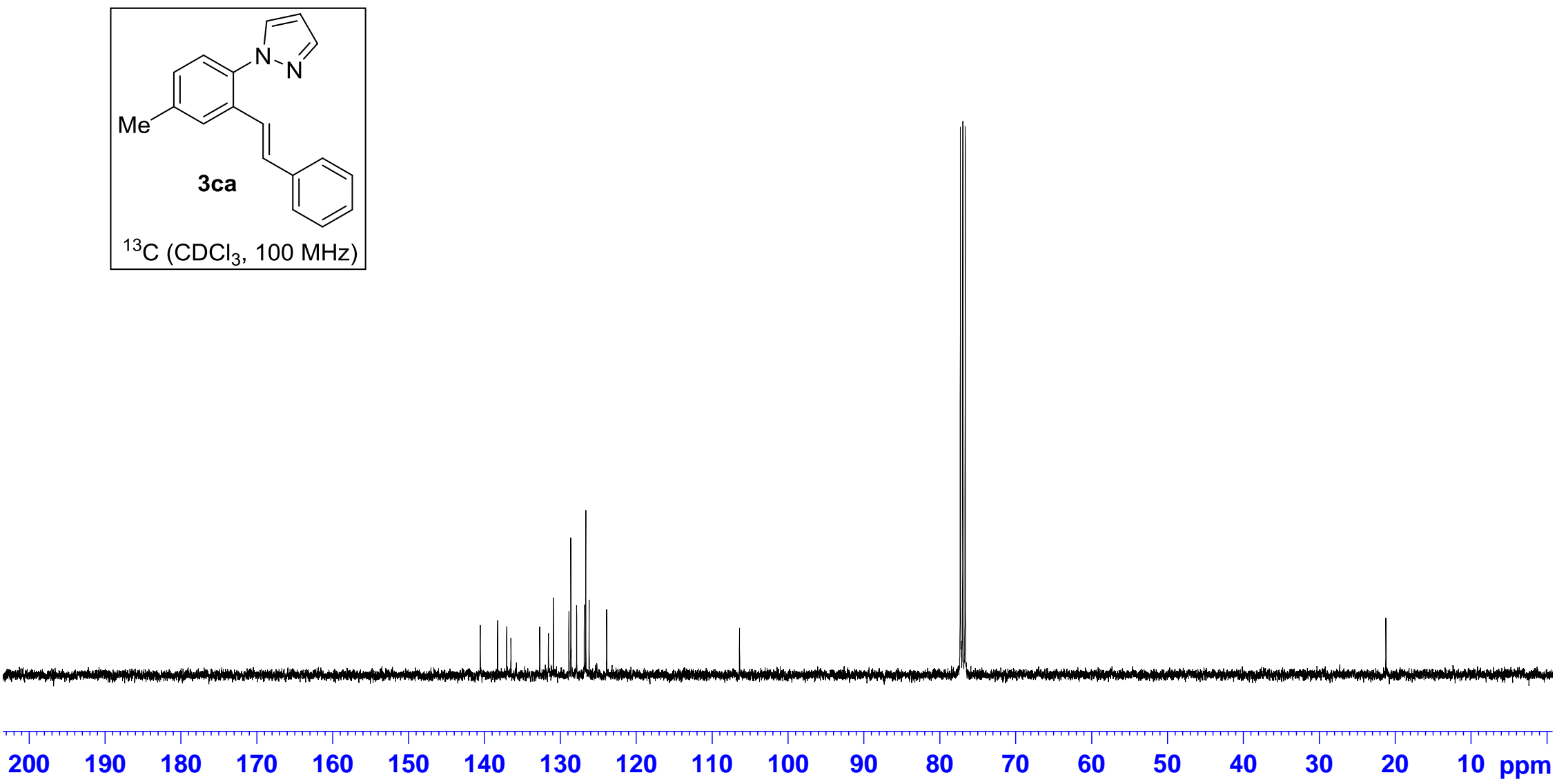


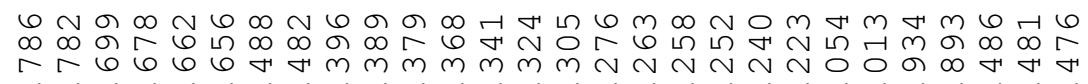

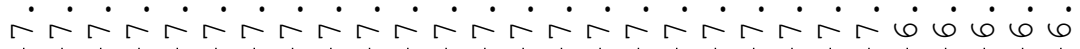

(nim
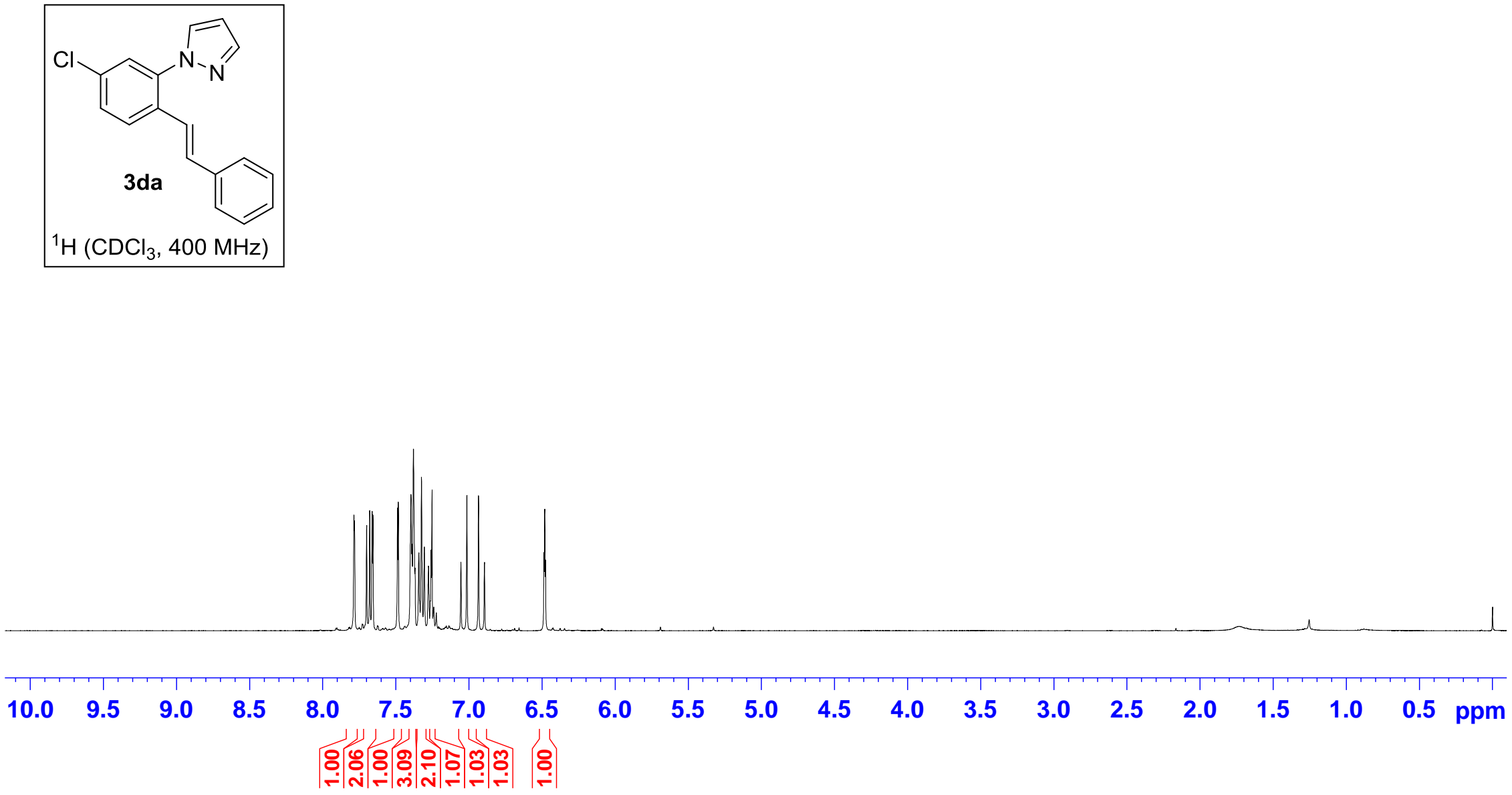

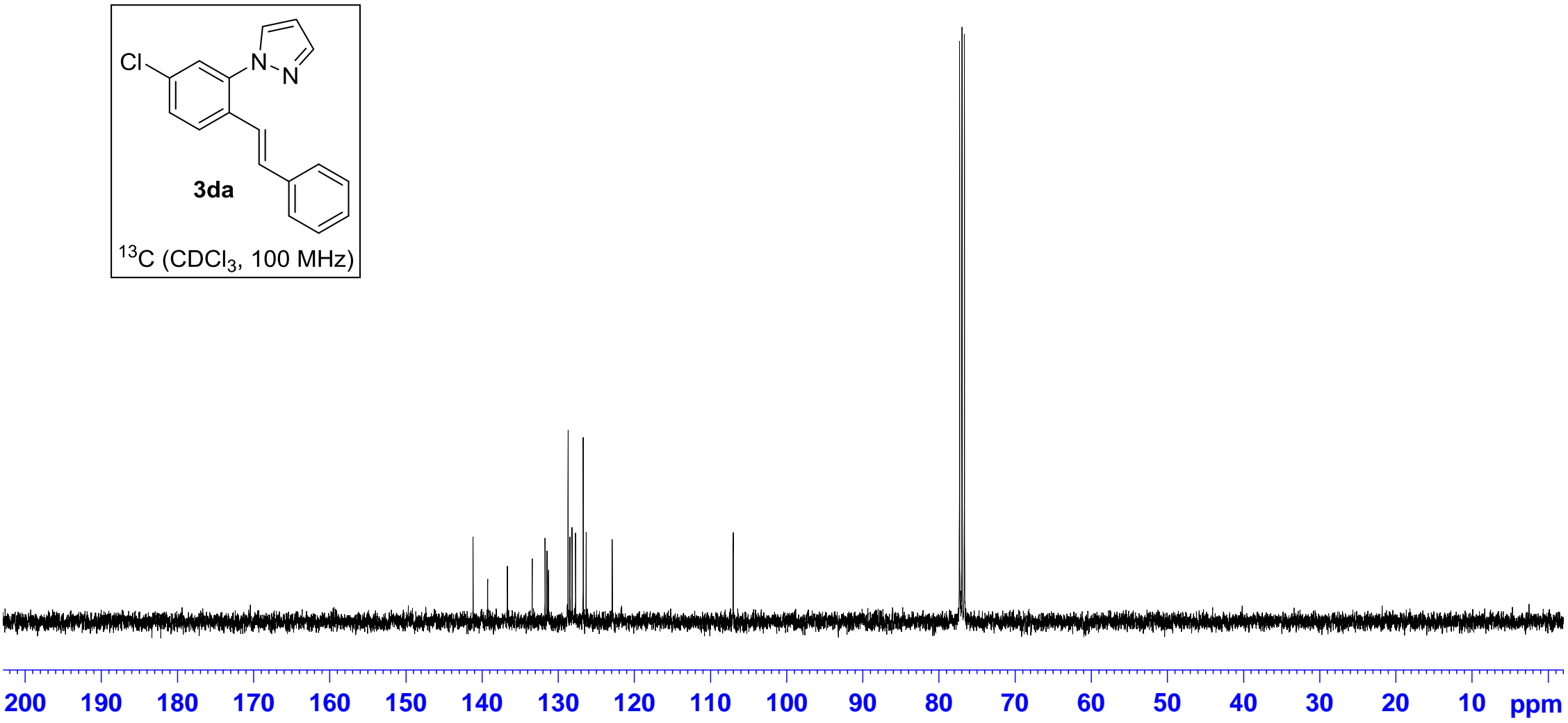


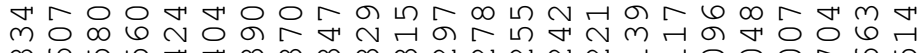
$6 r i r$
0.4
0

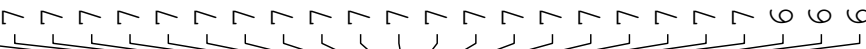
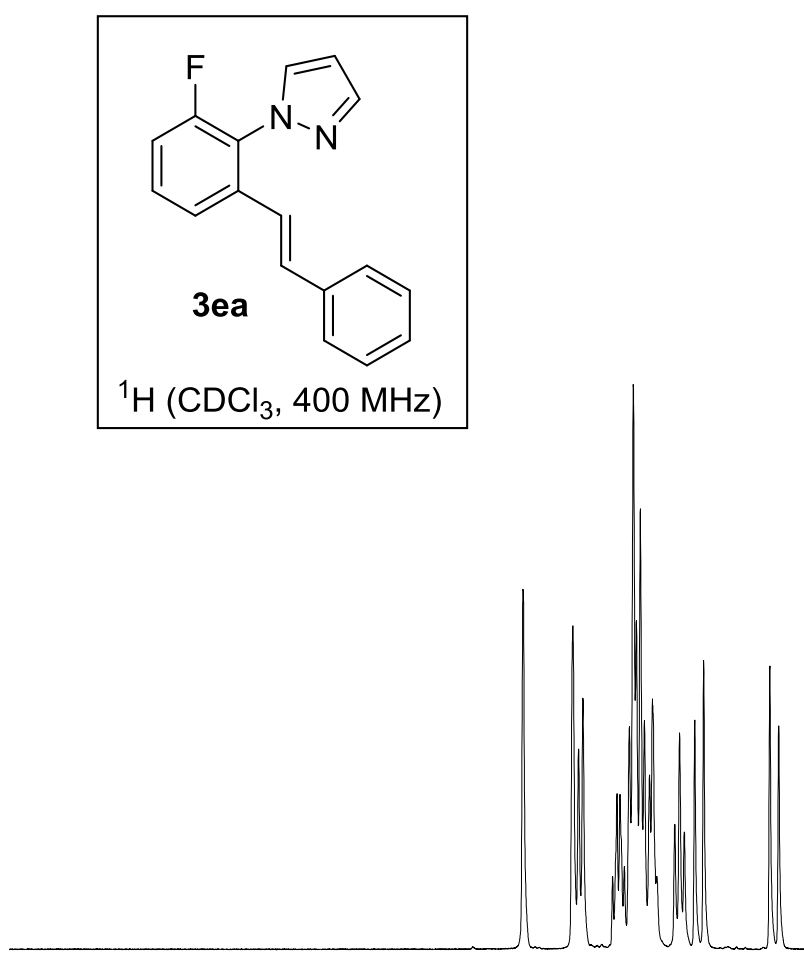

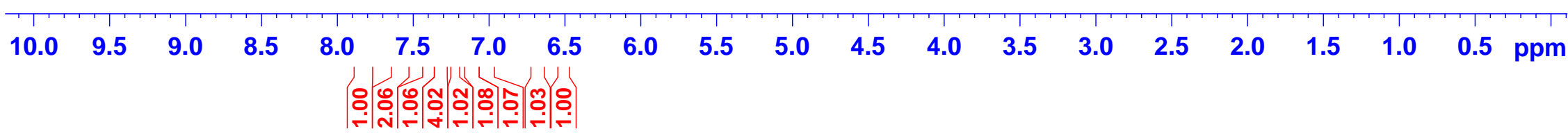




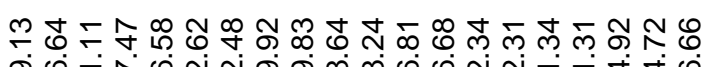

هin

1 这运
개요요

사잉

I/
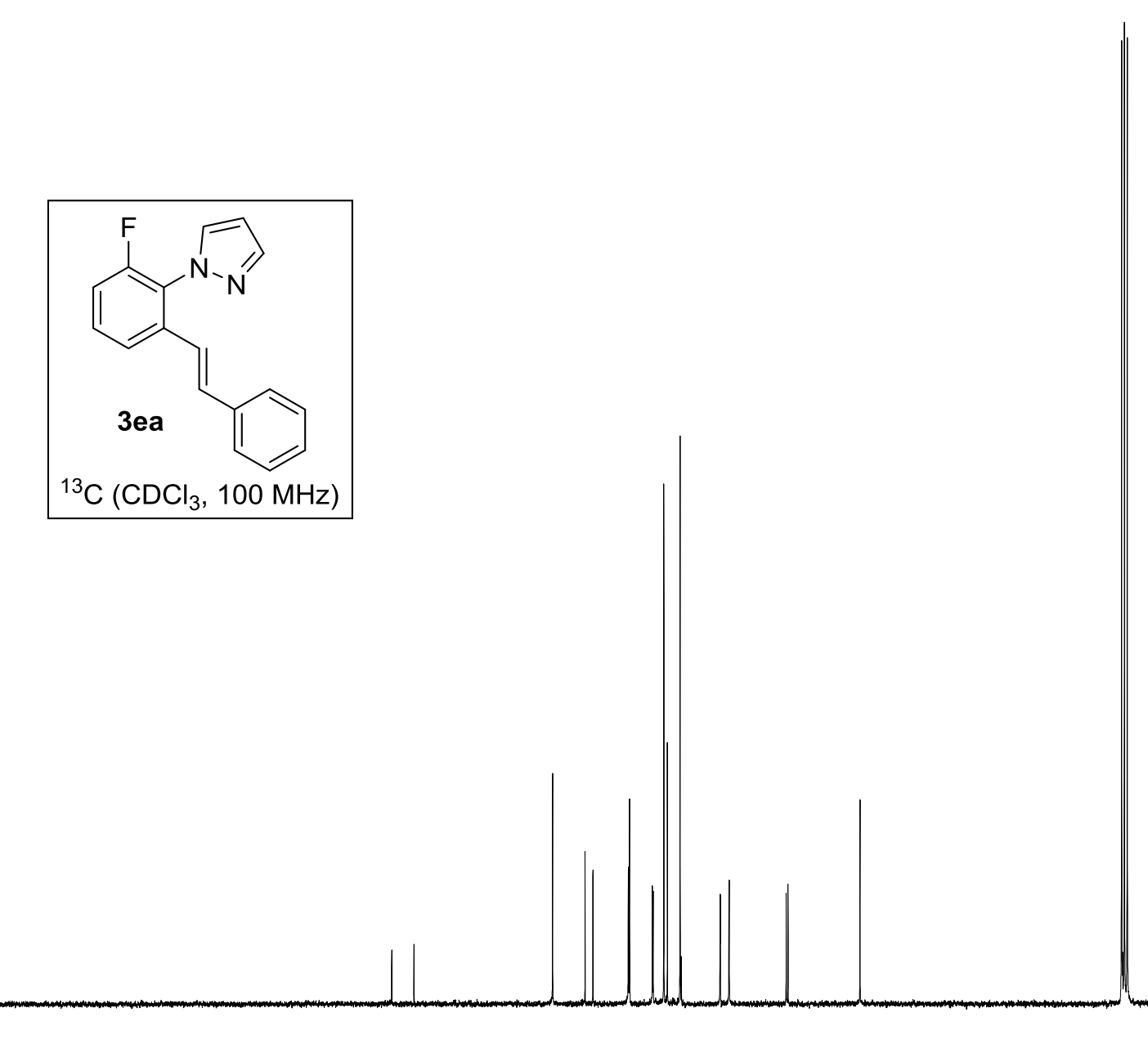

200

190180

$180 \quad 170$

160150

$140 \quad 130$

$120 \quad 110 \quad 100$

90

80

6050

$50 \quad 40$

4030

$30 \quad 20$

$2010 \mathrm{ppm}$ 

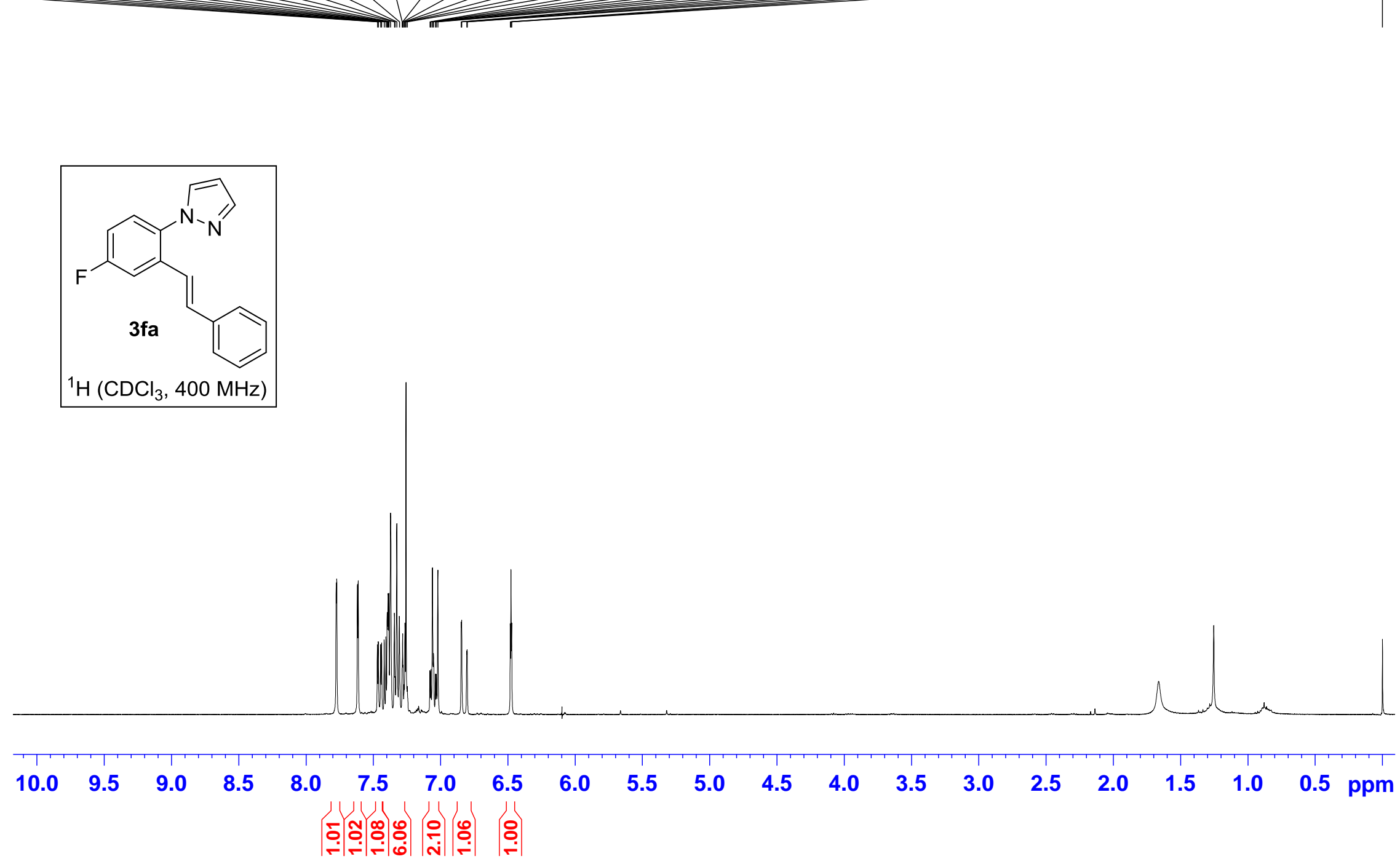


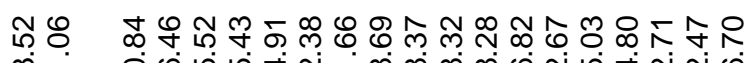

ஜ்

1

\section{$\bar{m} 8 \%$}

상

Y
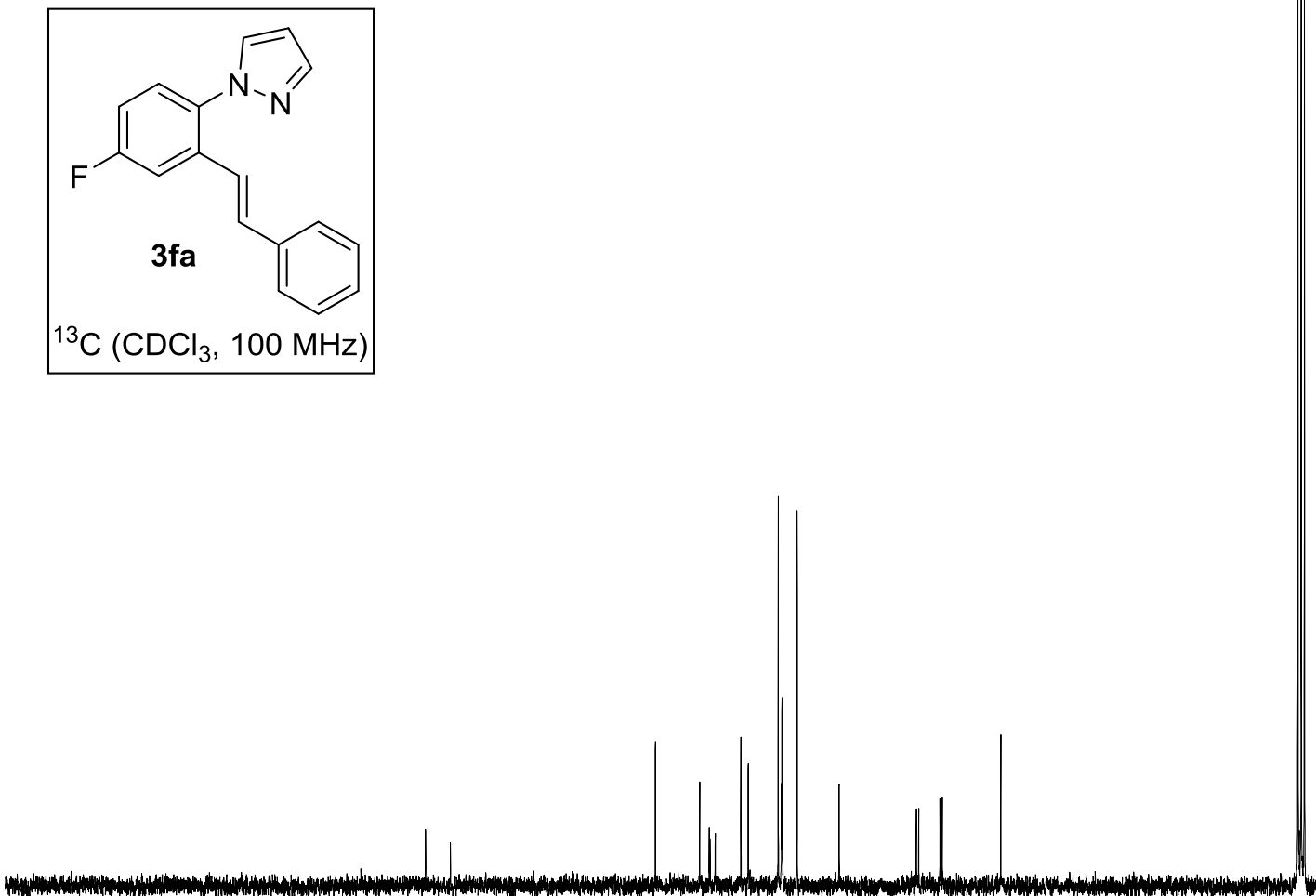

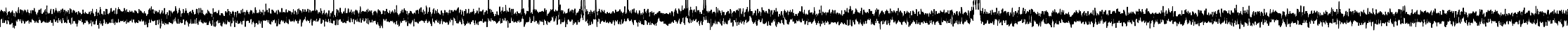

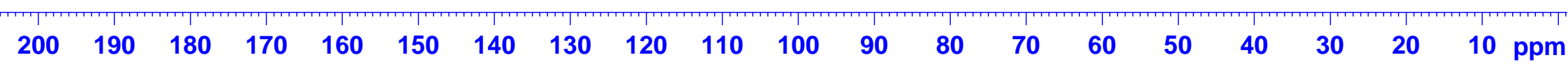




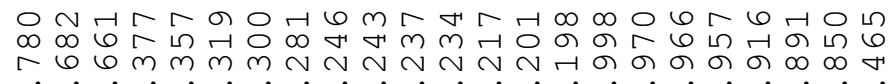

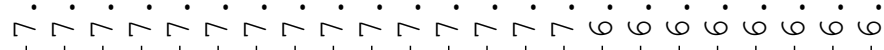

$\longrightarrow$
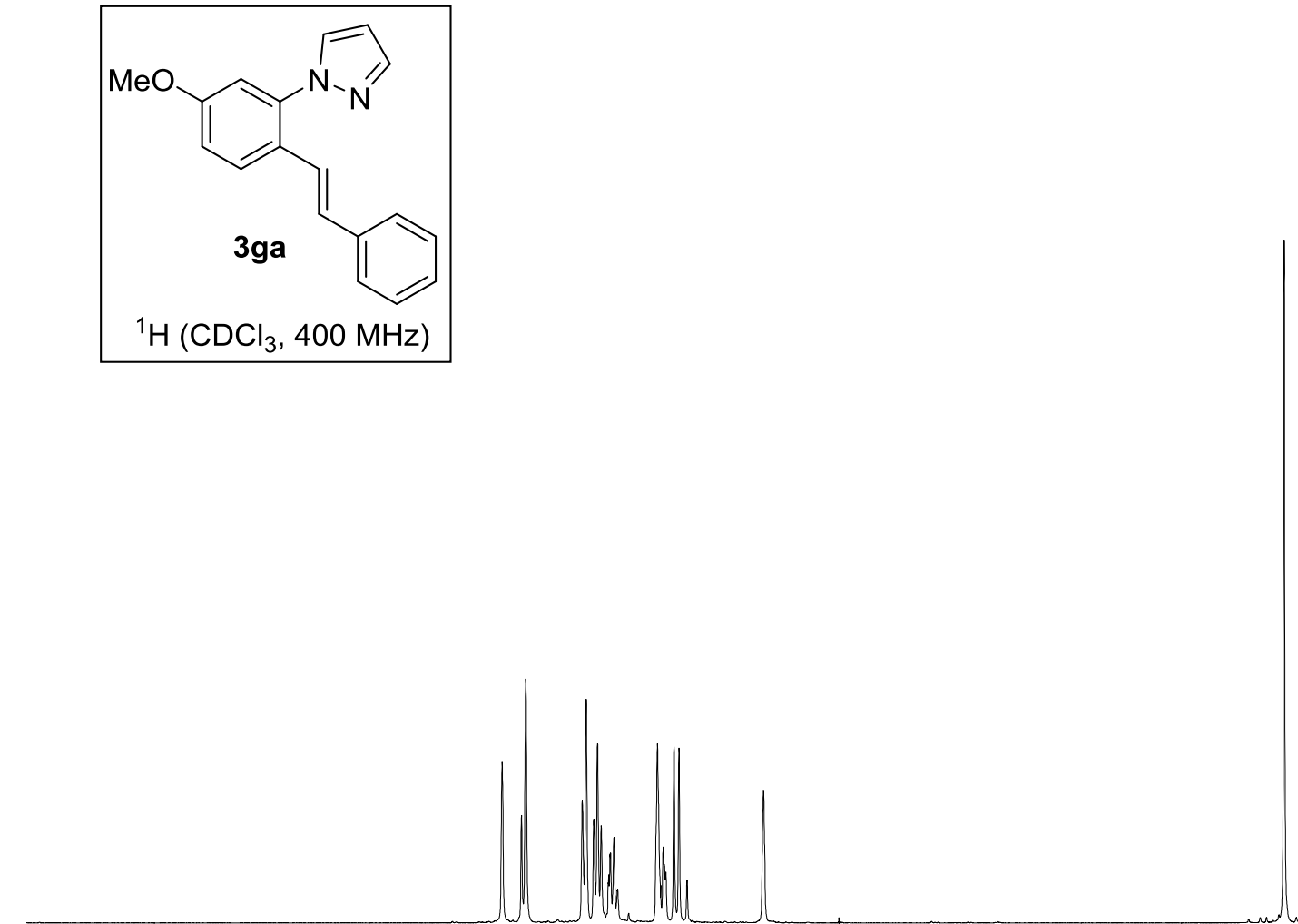

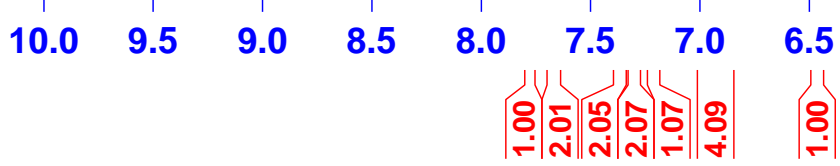

$\begin{array}{lllllllllllll}6.0 & 5.5 & 5.0 & 4.5 & 4.0 & 3.5 & 3.0 & 2.5 & 2.0 & 1.5 & 1.0 & 0.5 & \text { ppm }\end{array}$ 

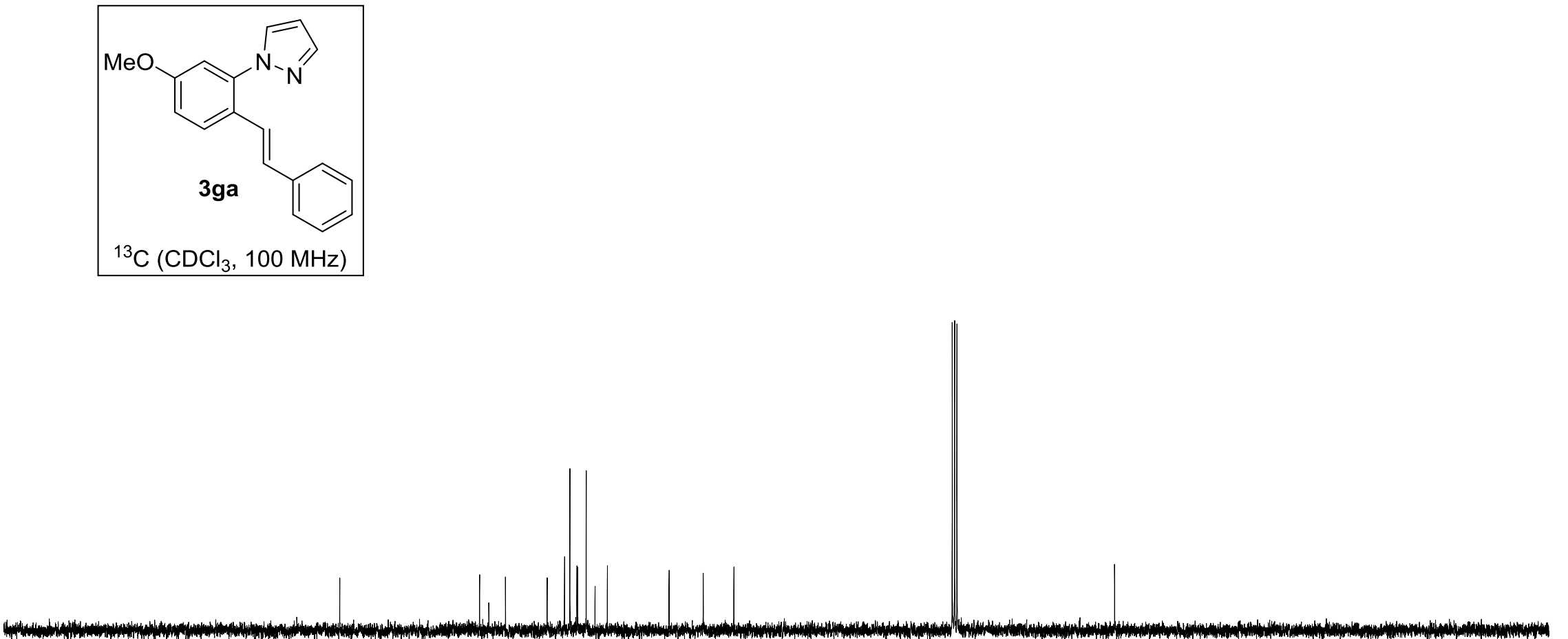


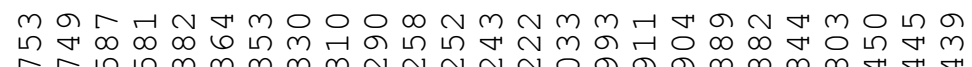

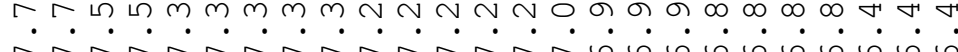

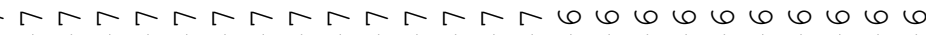

$\longrightarrow$
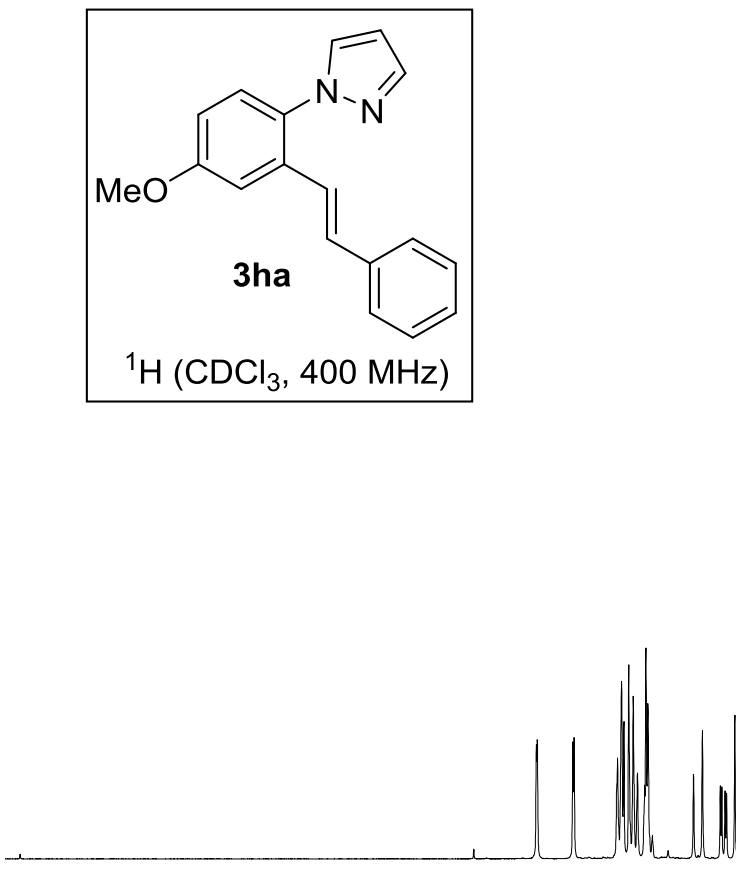

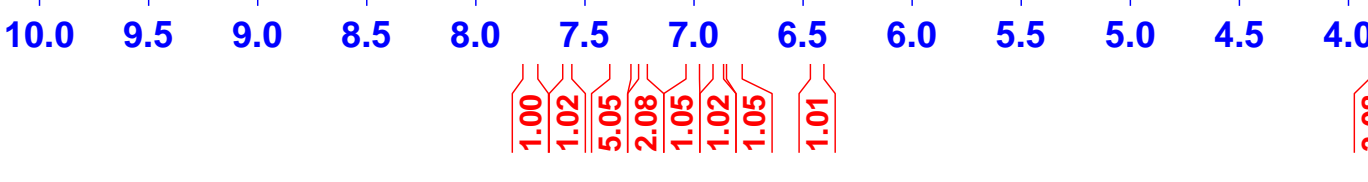




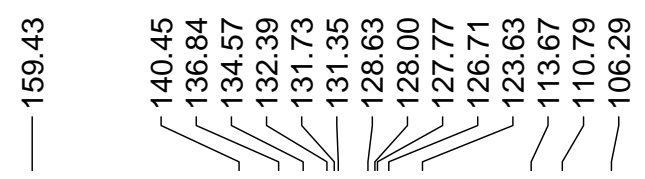

लㄷㅇㅇㅛ

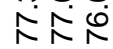

8

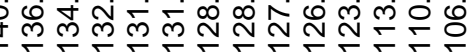
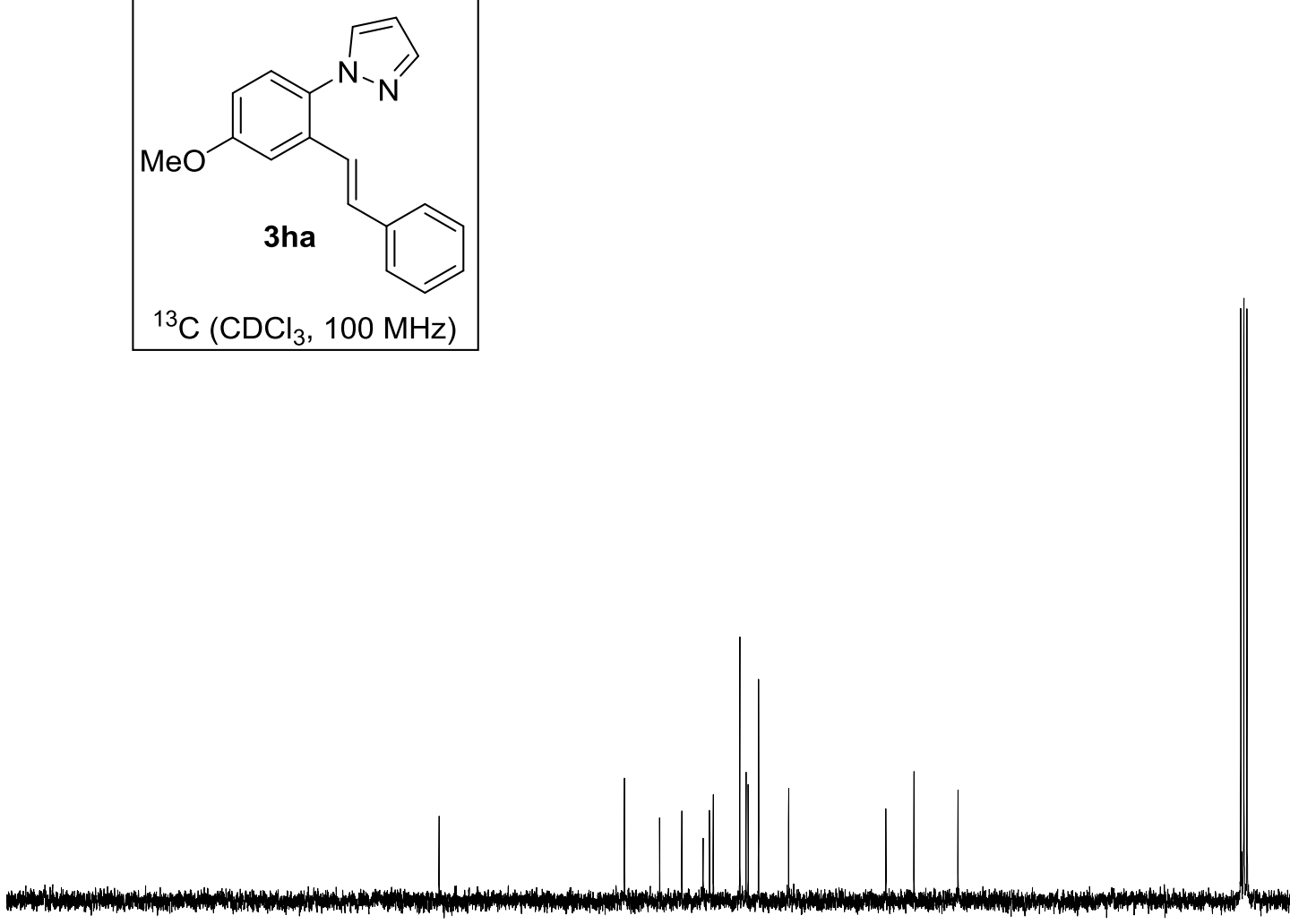
(7)
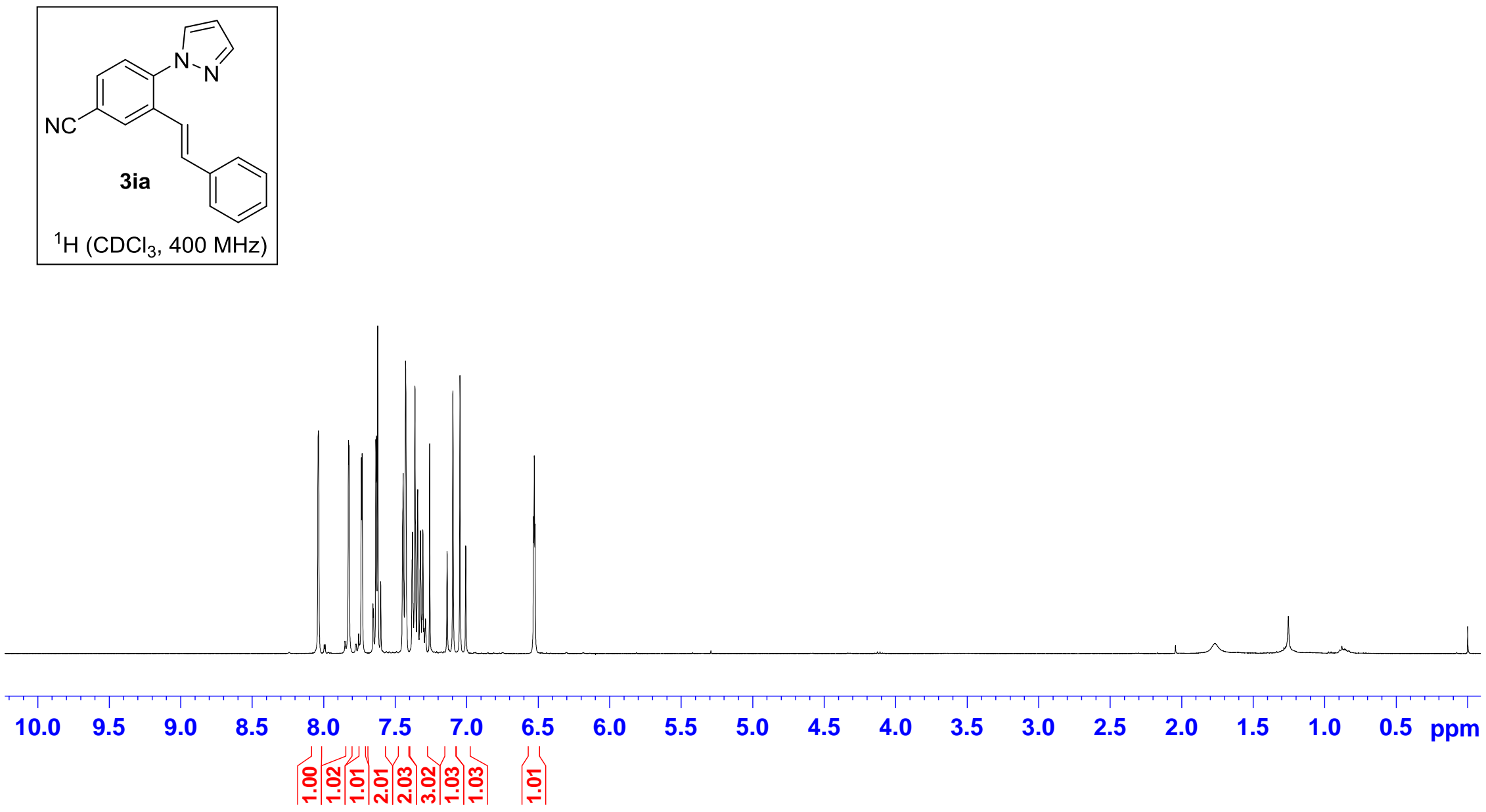


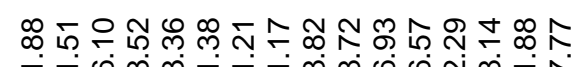

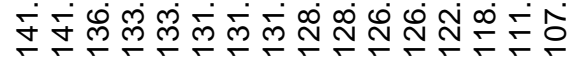

( m다

상

I/

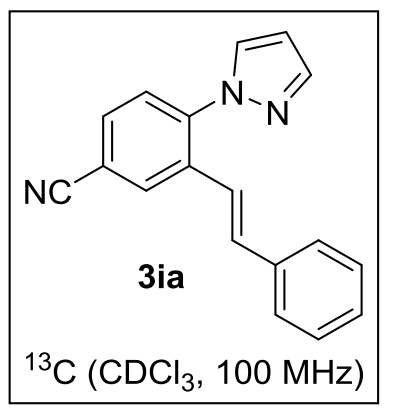

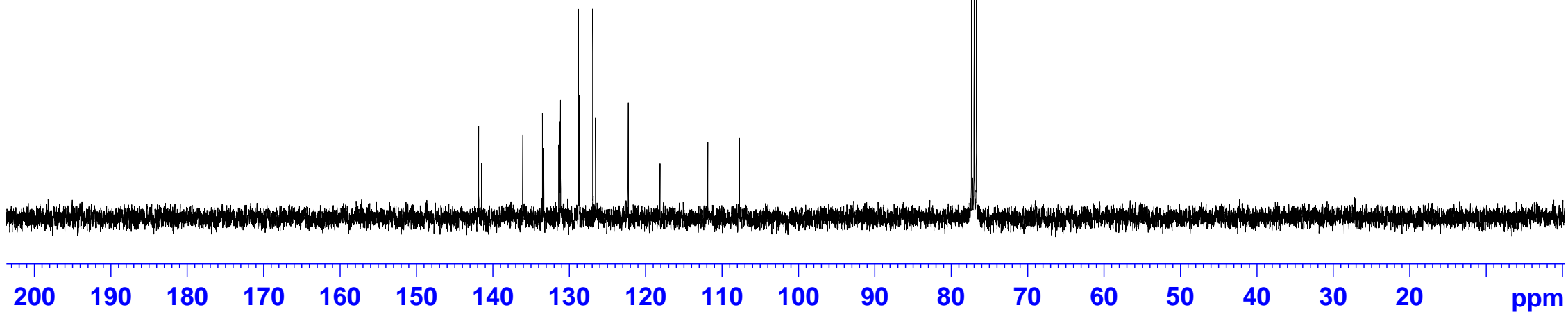




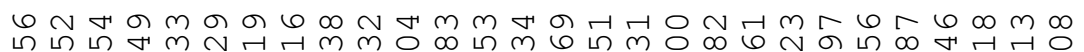
ம

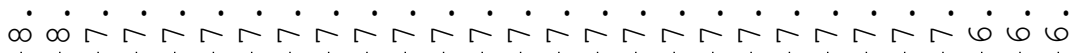
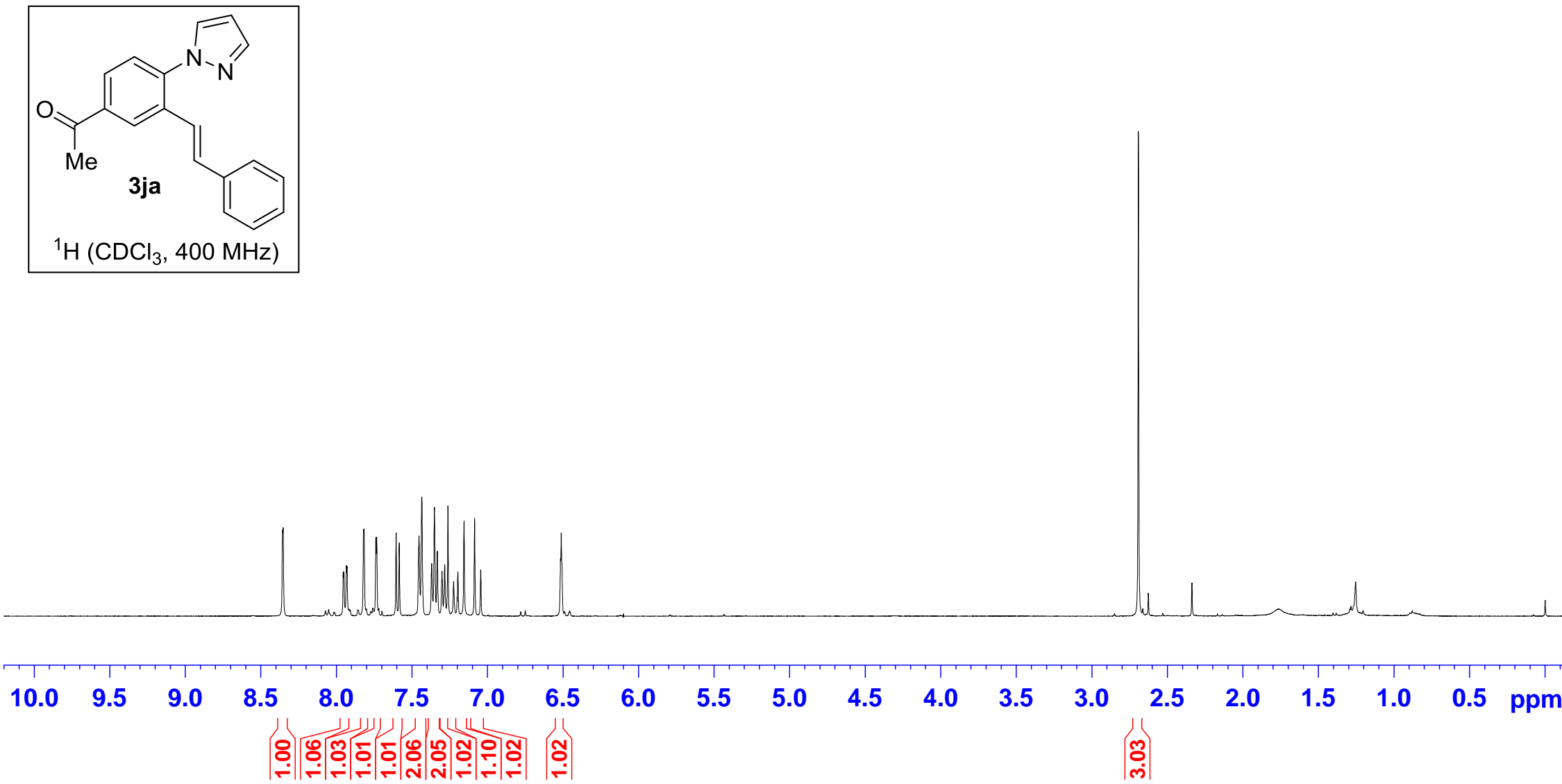


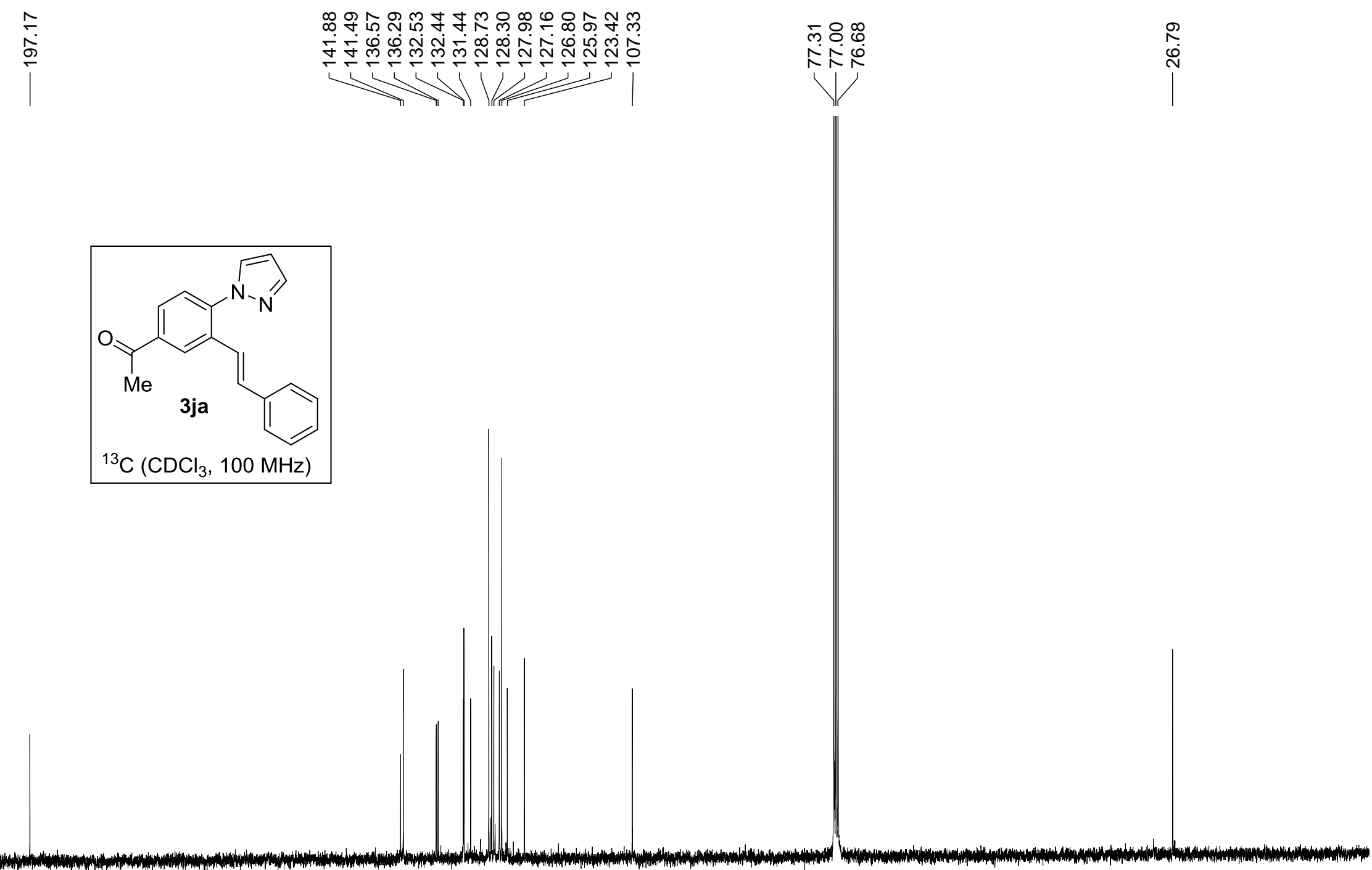

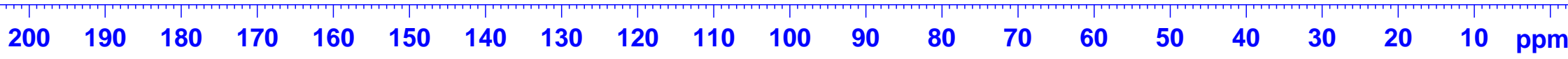



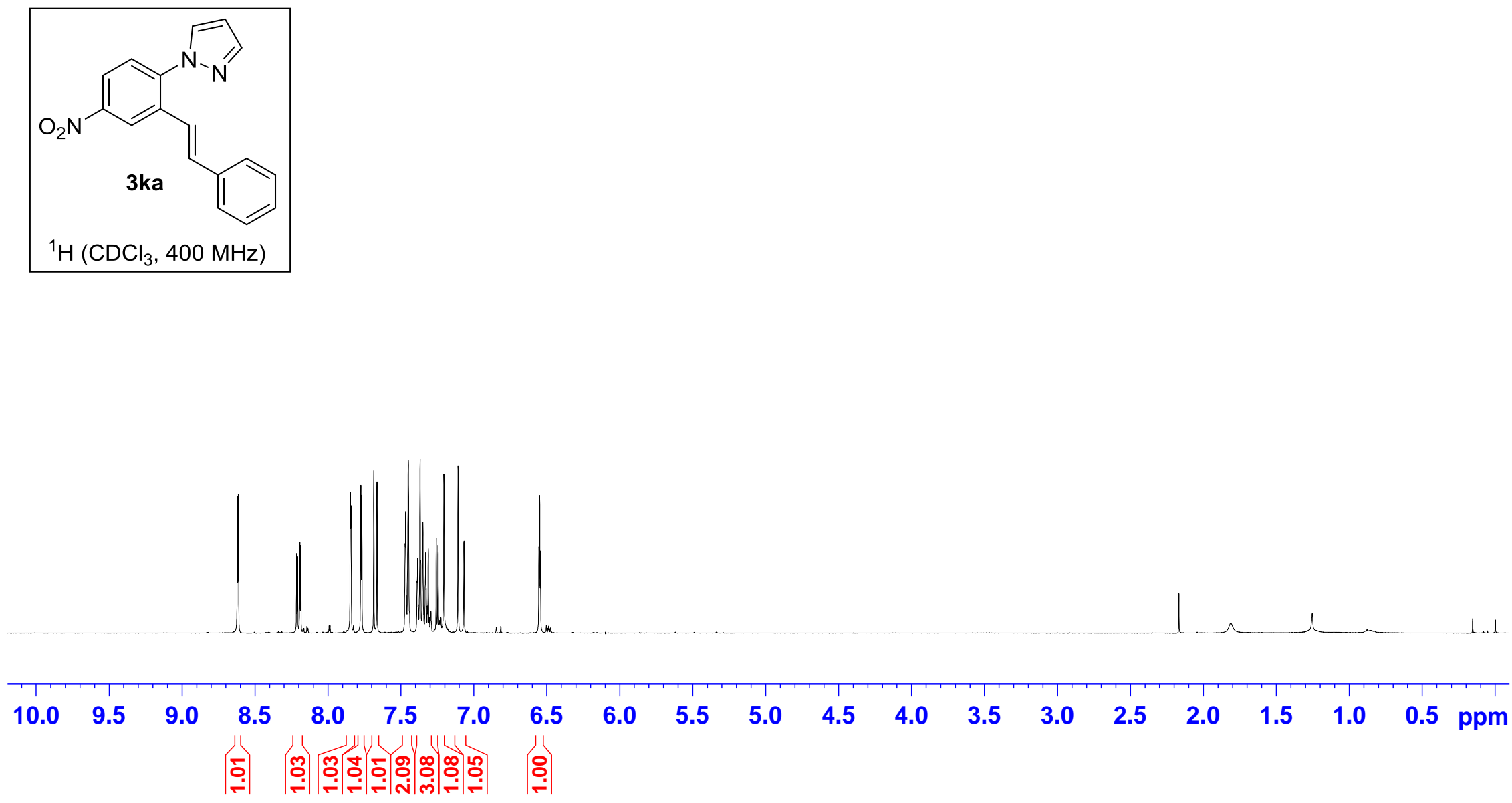

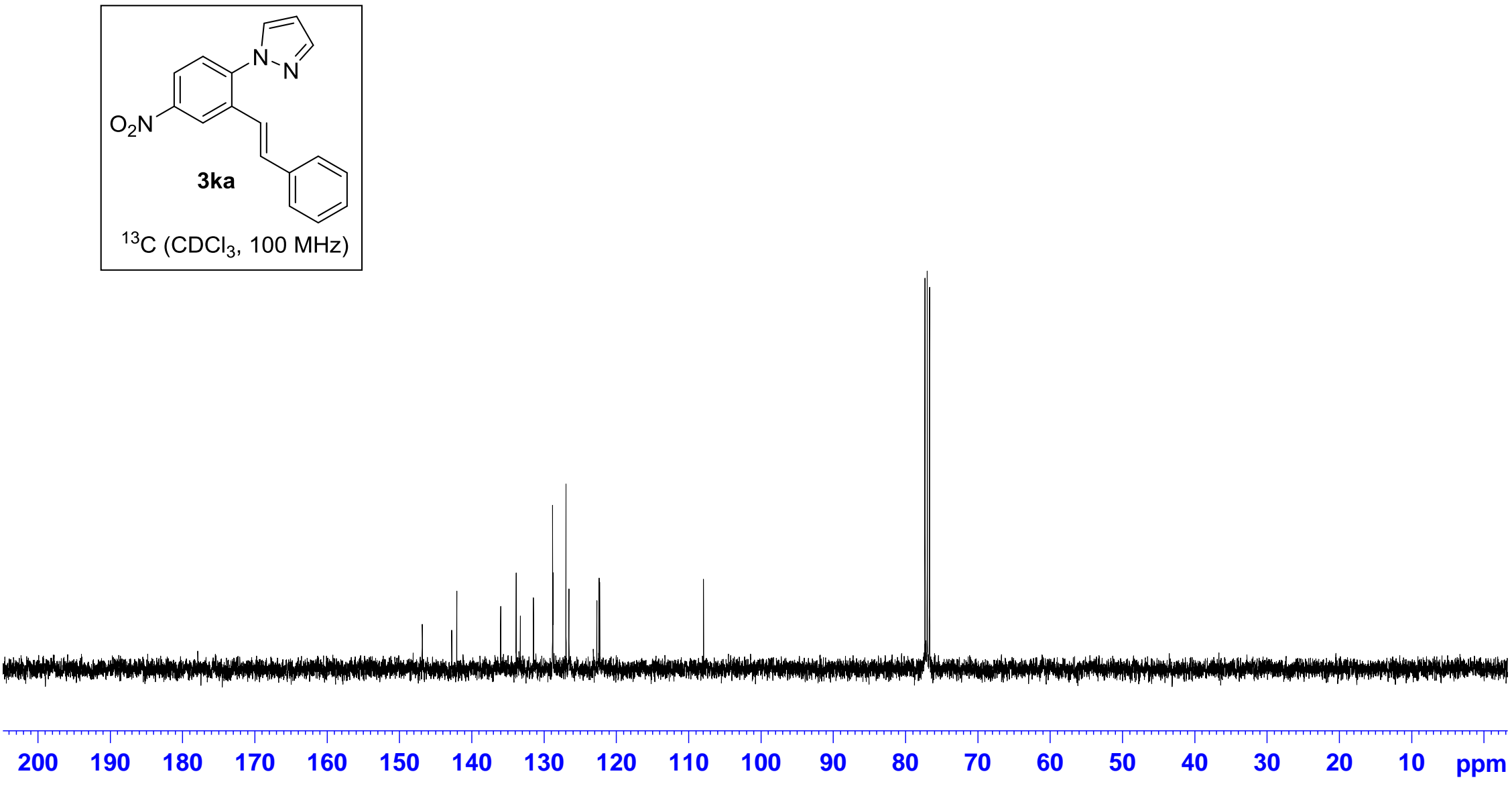

100

9080

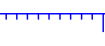

$\begin{array}{lllllll}60 & 50 & 40 & 30 & 20 & 10 & \mathrm{ppm}\end{array}$




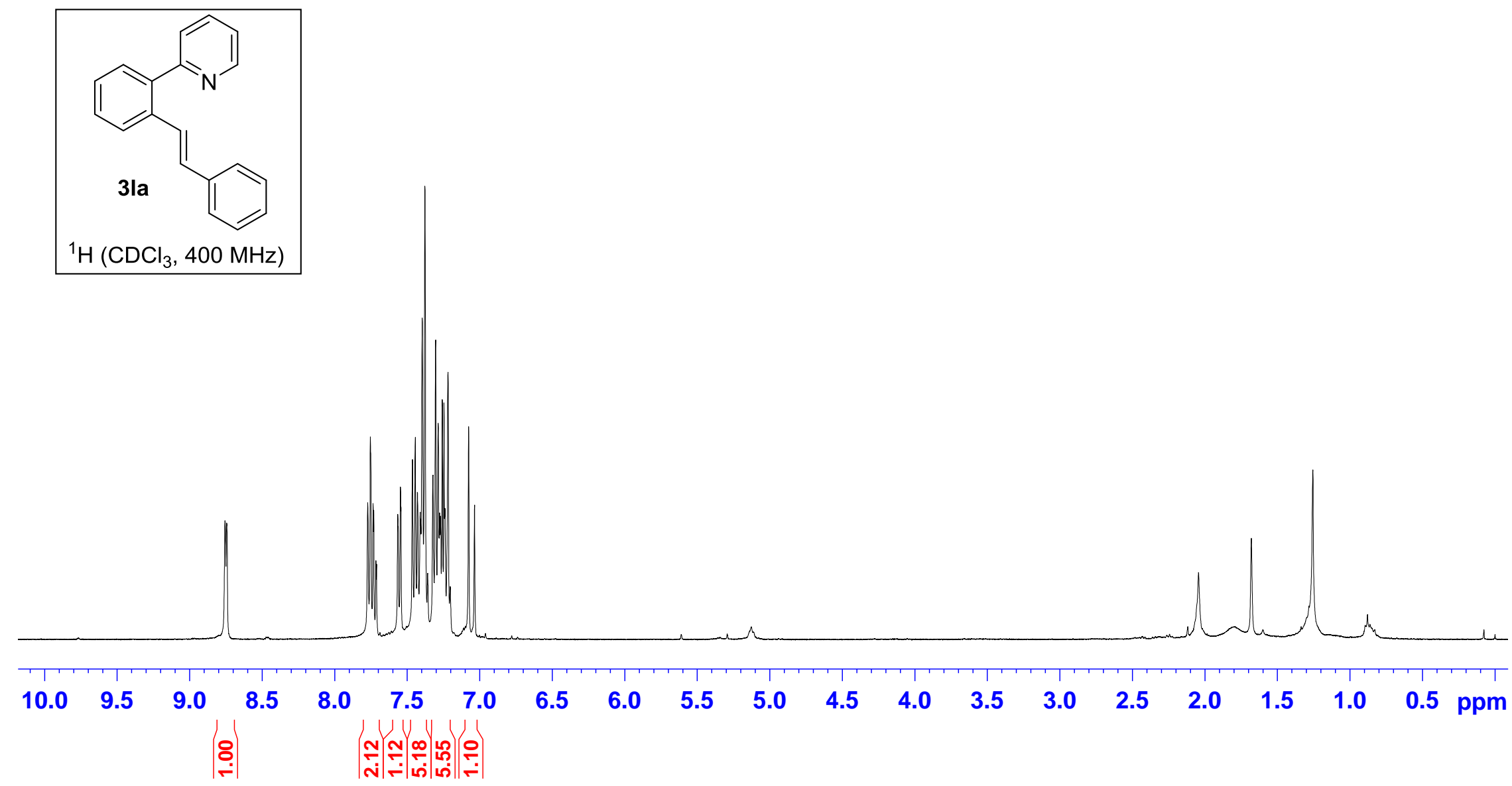



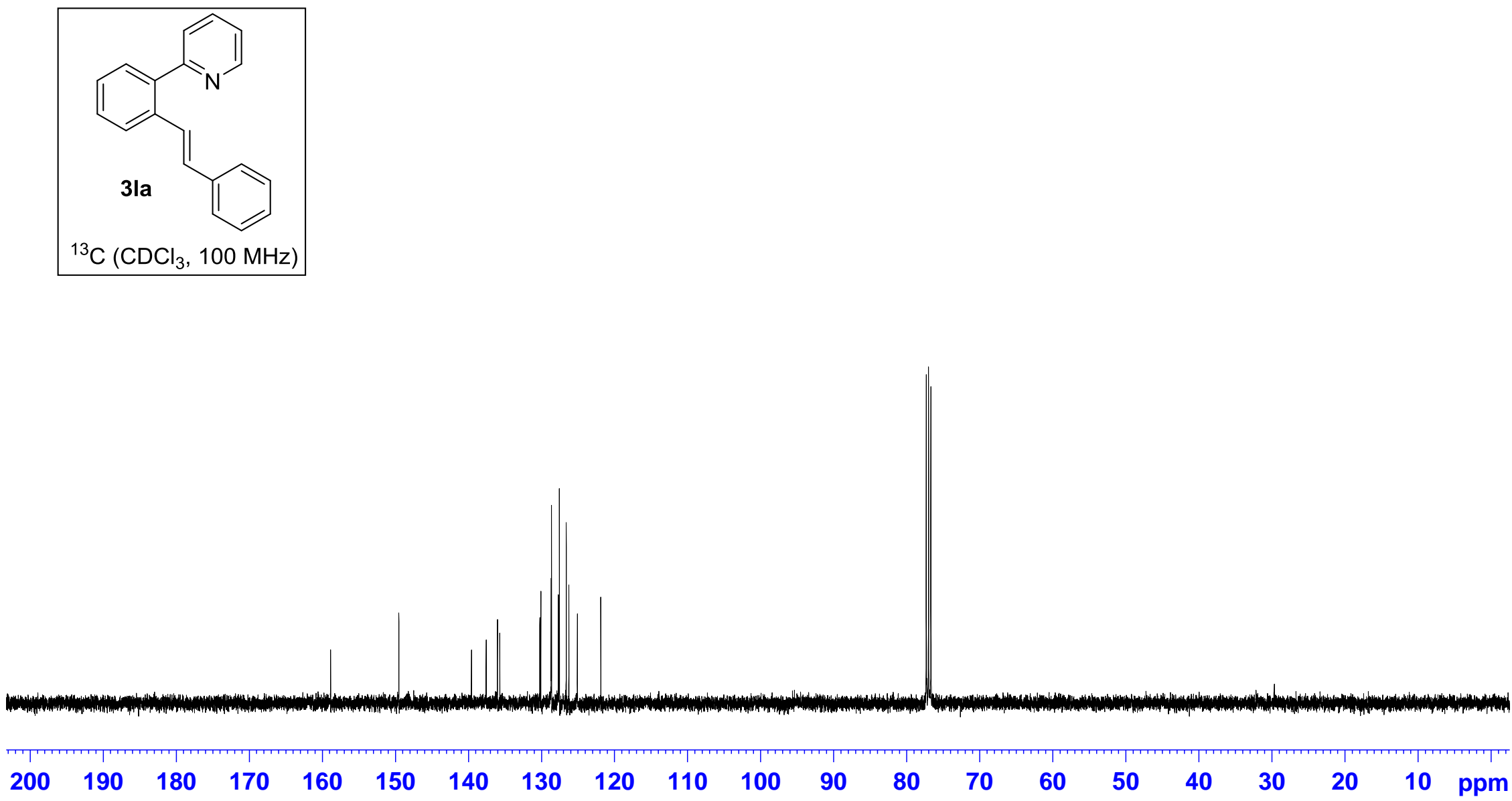

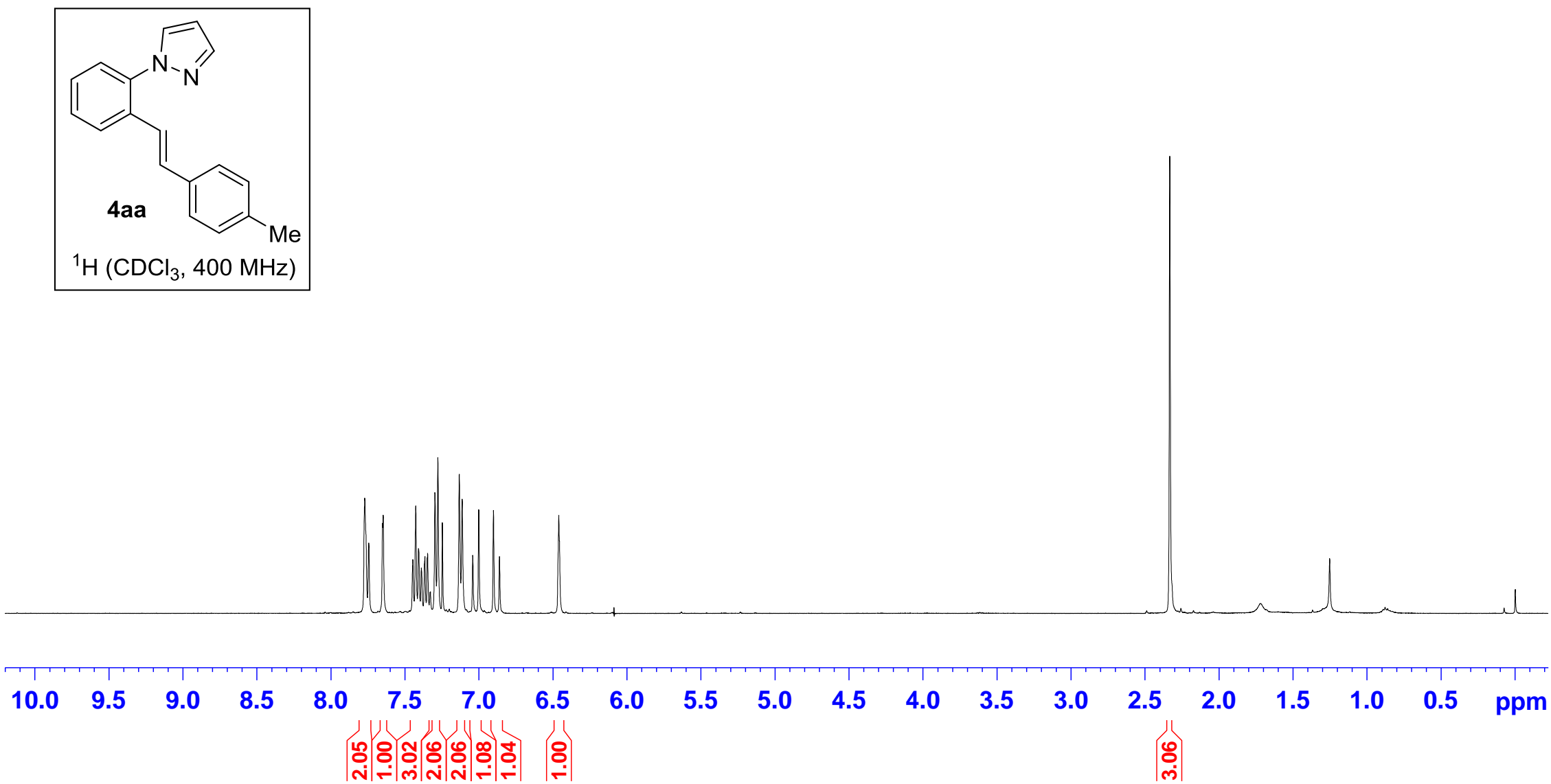

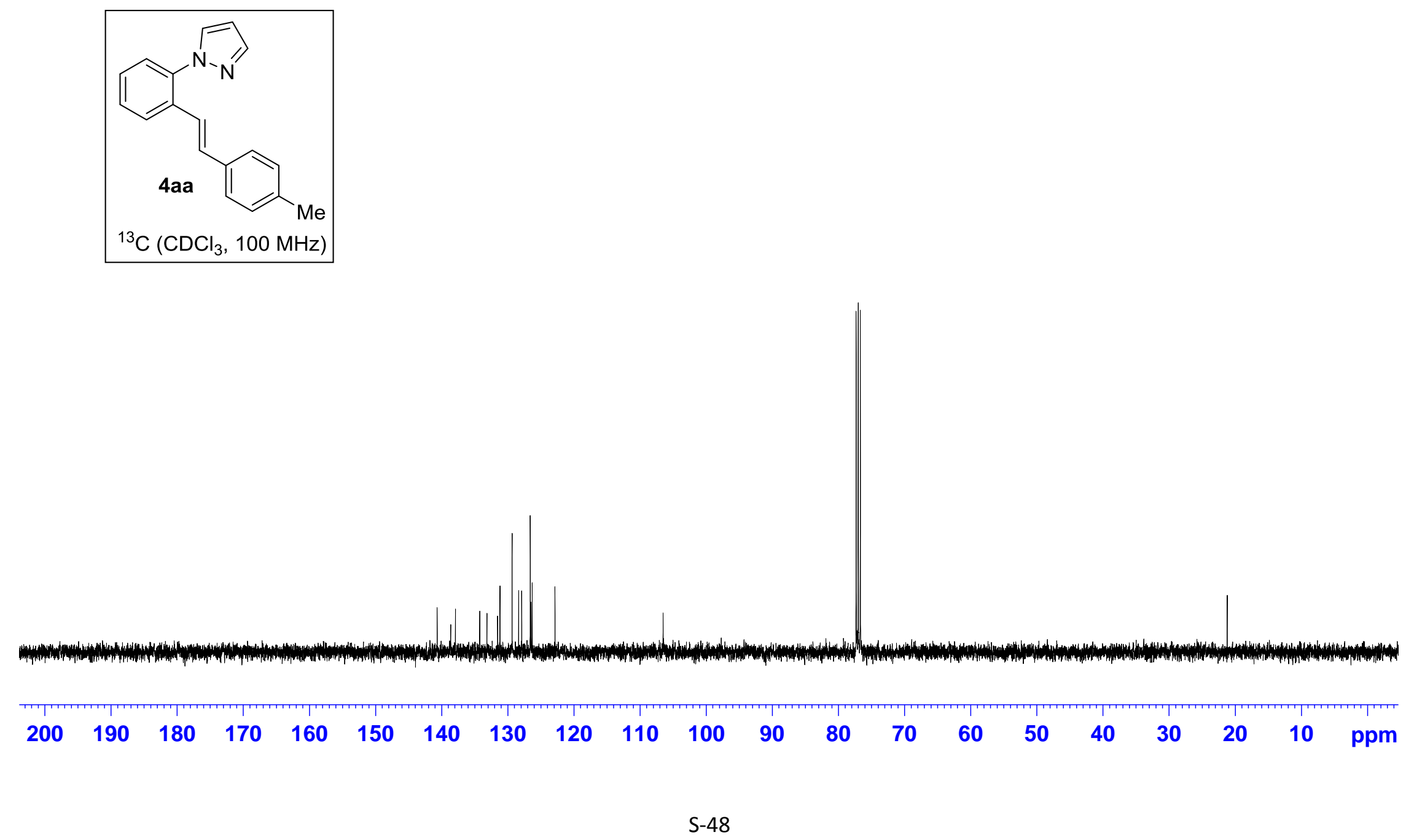


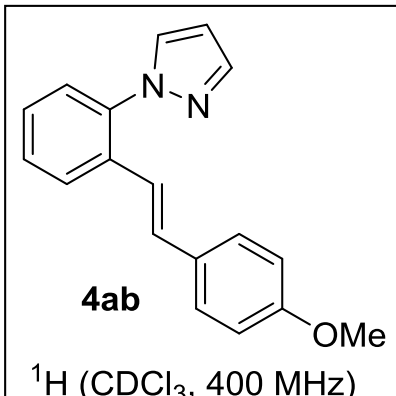

${ }^{1} \mathrm{H}\left(\mathrm{CDCl}_{3}, 400 \mathrm{MHz}\right)$

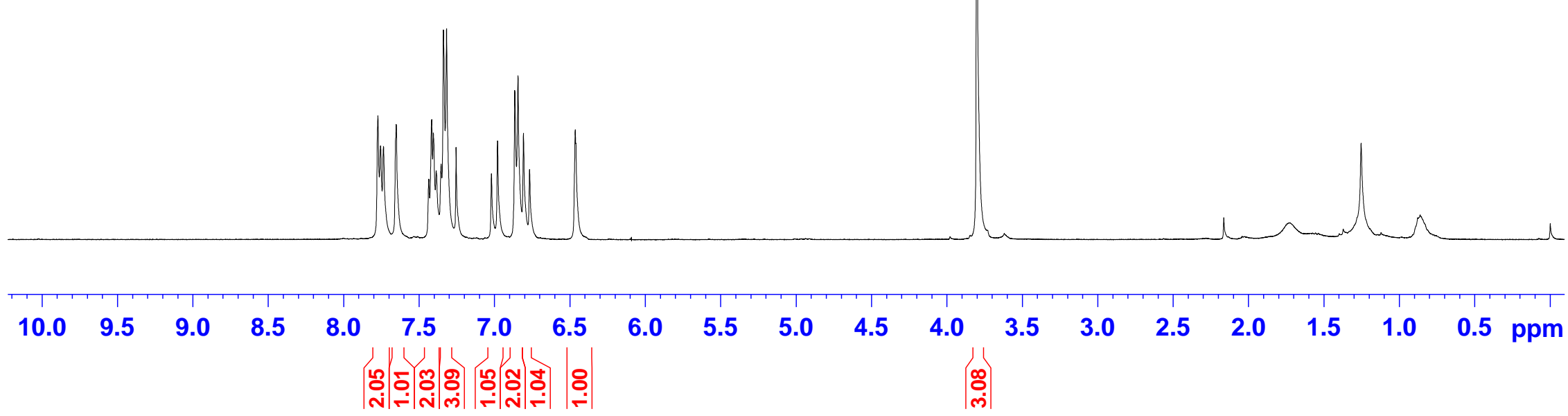



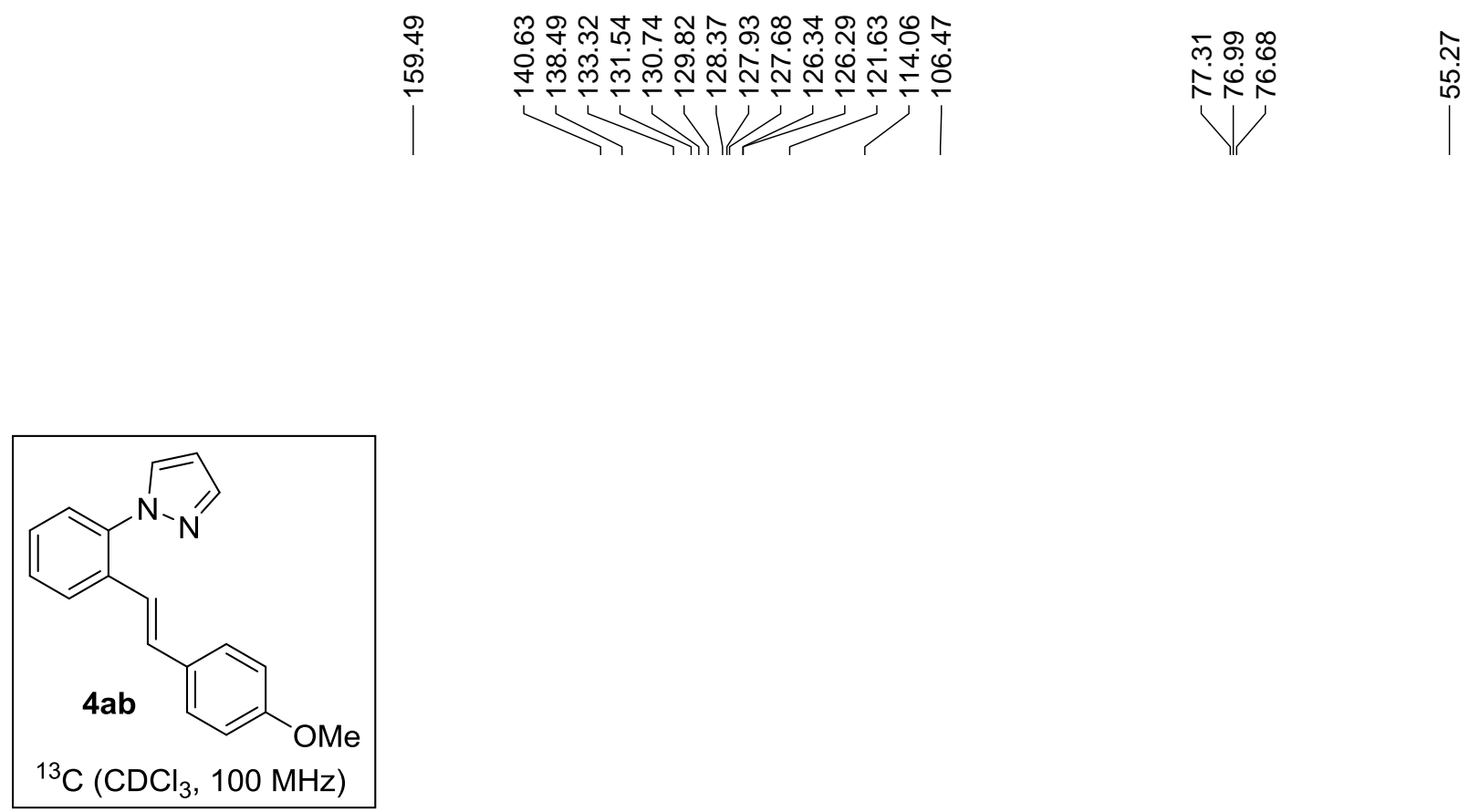

m.

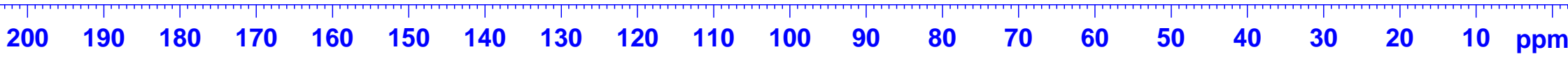




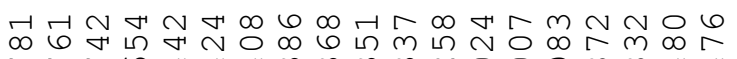

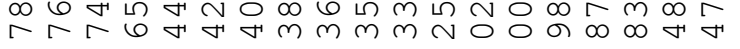

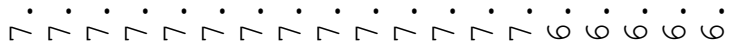

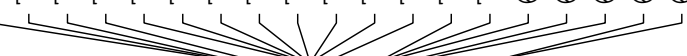
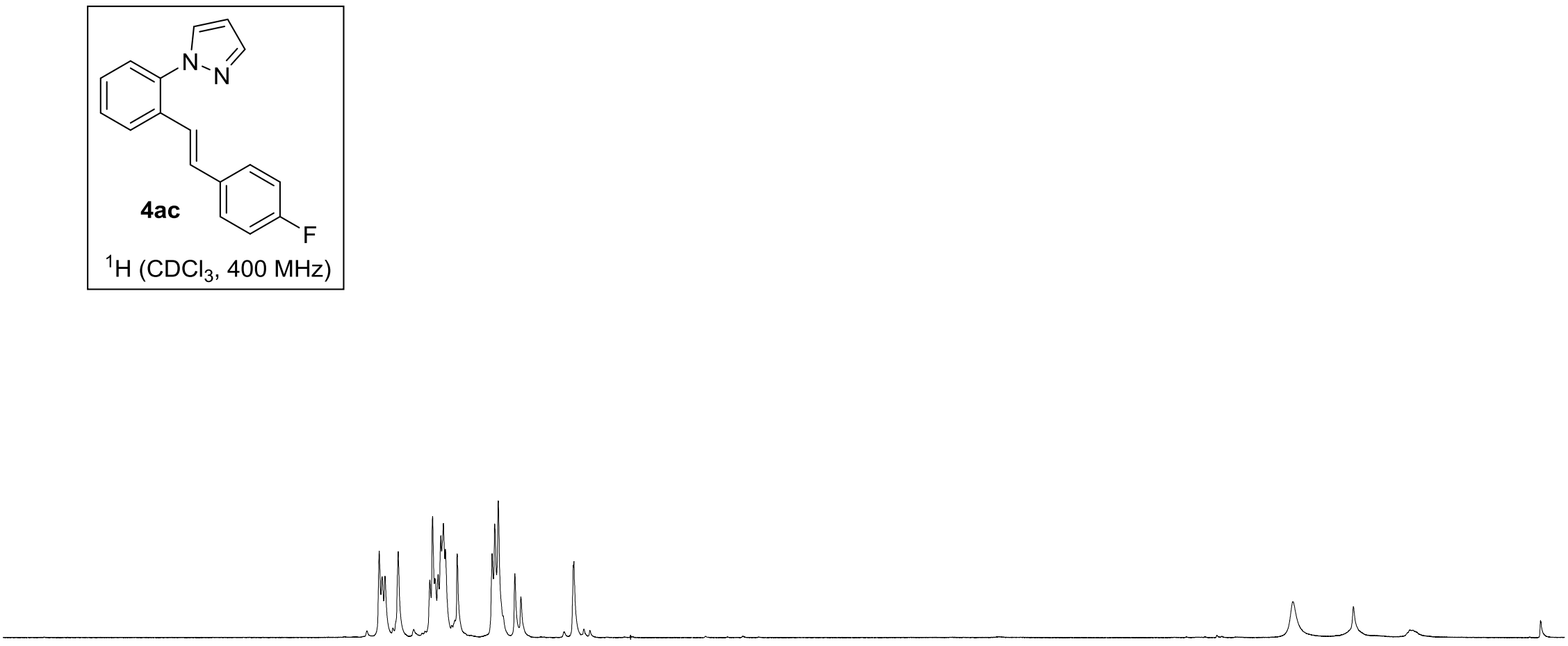

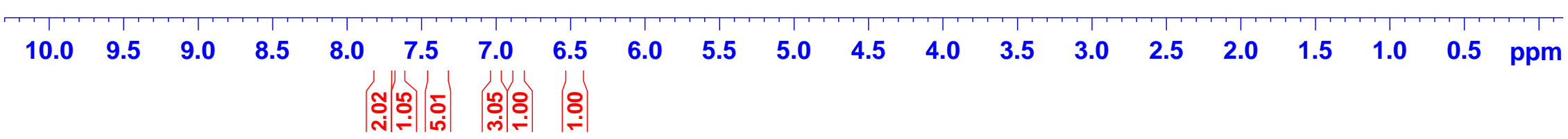




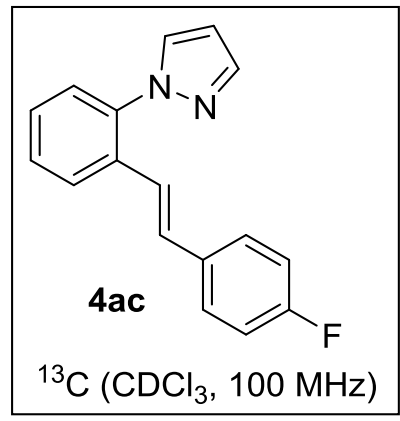

${ }^{3} \mathrm{C}\left(\mathrm{CDCl}_{3}, 100 \mathrm{MHz}\right)$

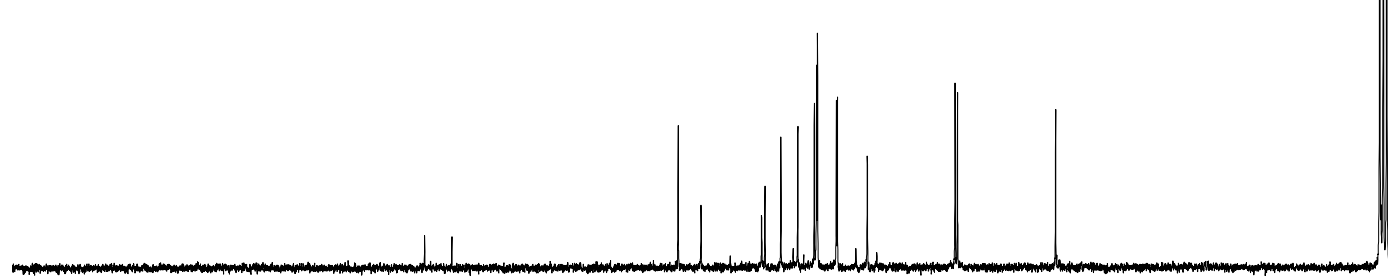

190

$180 \quad 170$

150

140

130

120

110100

90

80

70

6050

50

4030

3020 


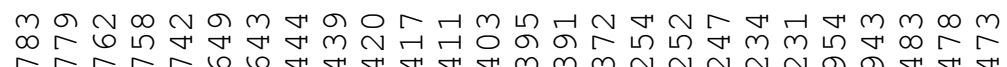

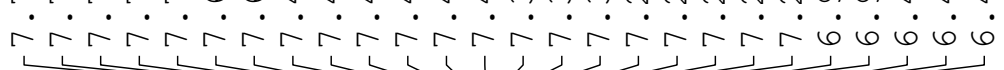
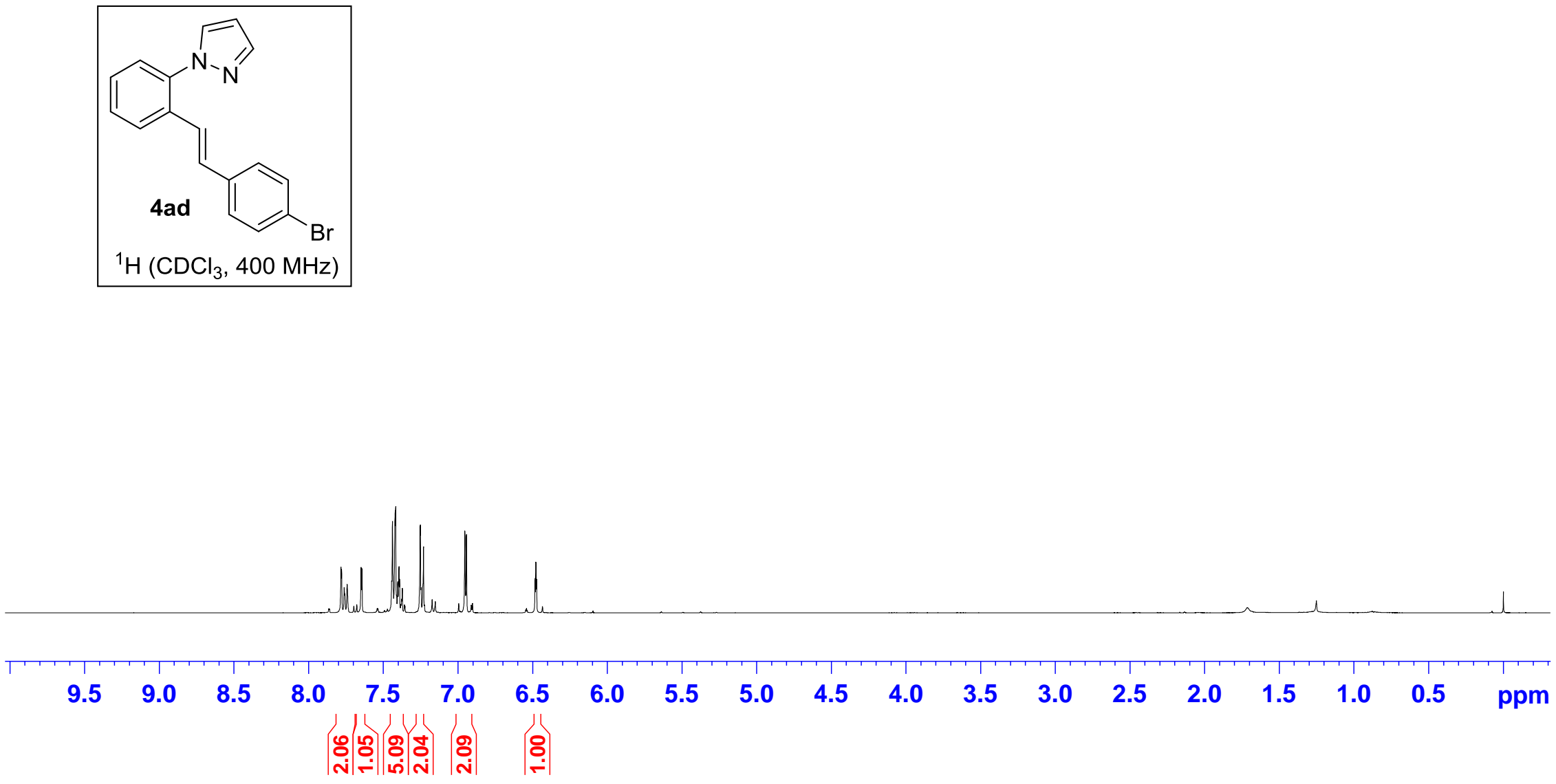


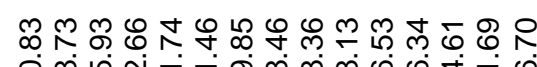

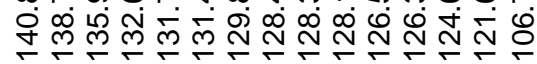

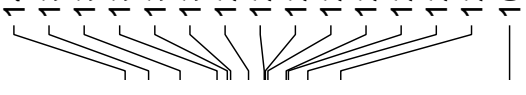

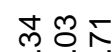

คำ

V
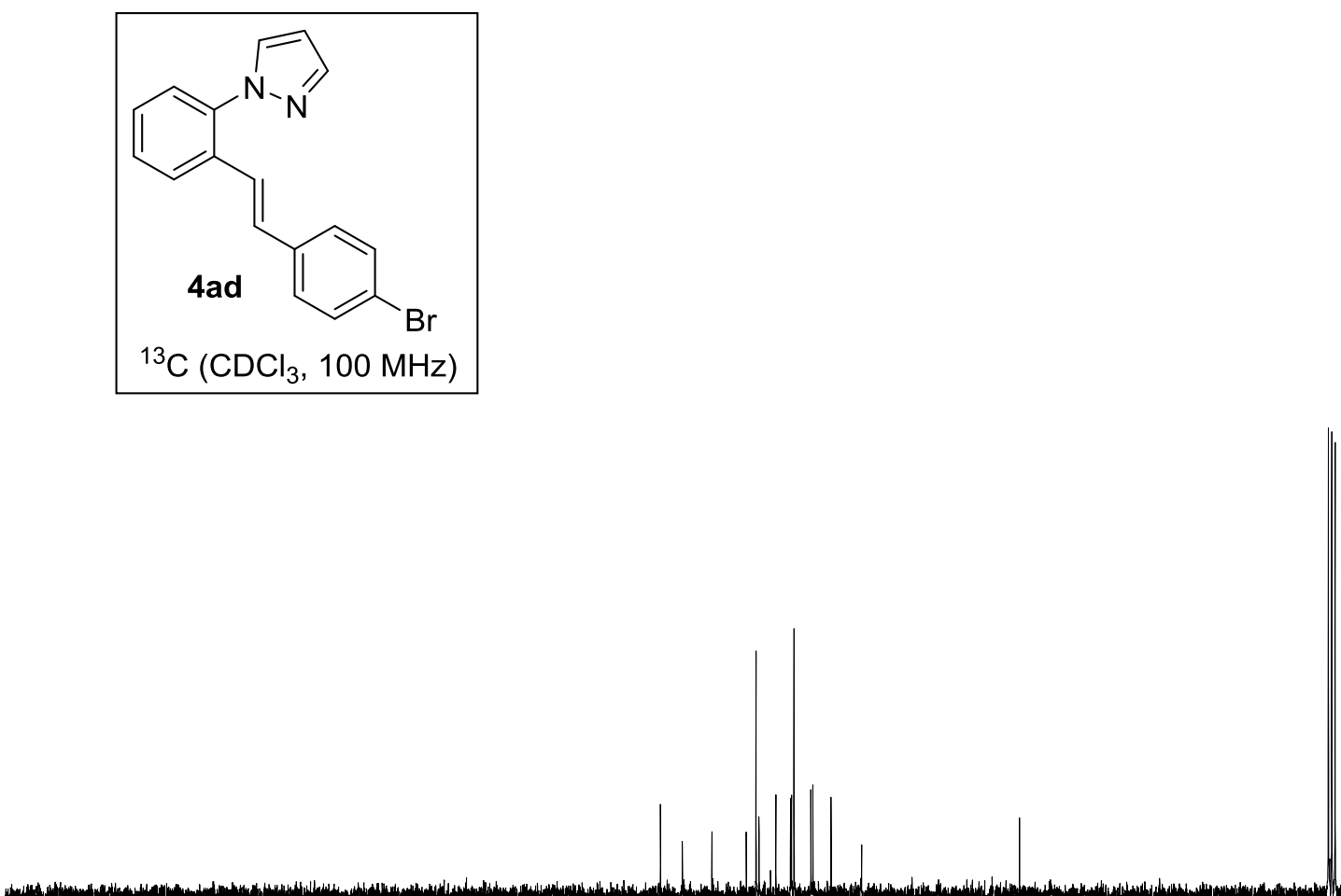
강ํํ

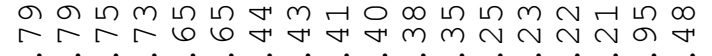

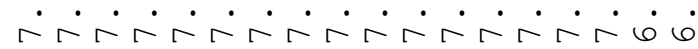

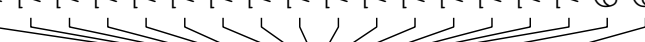

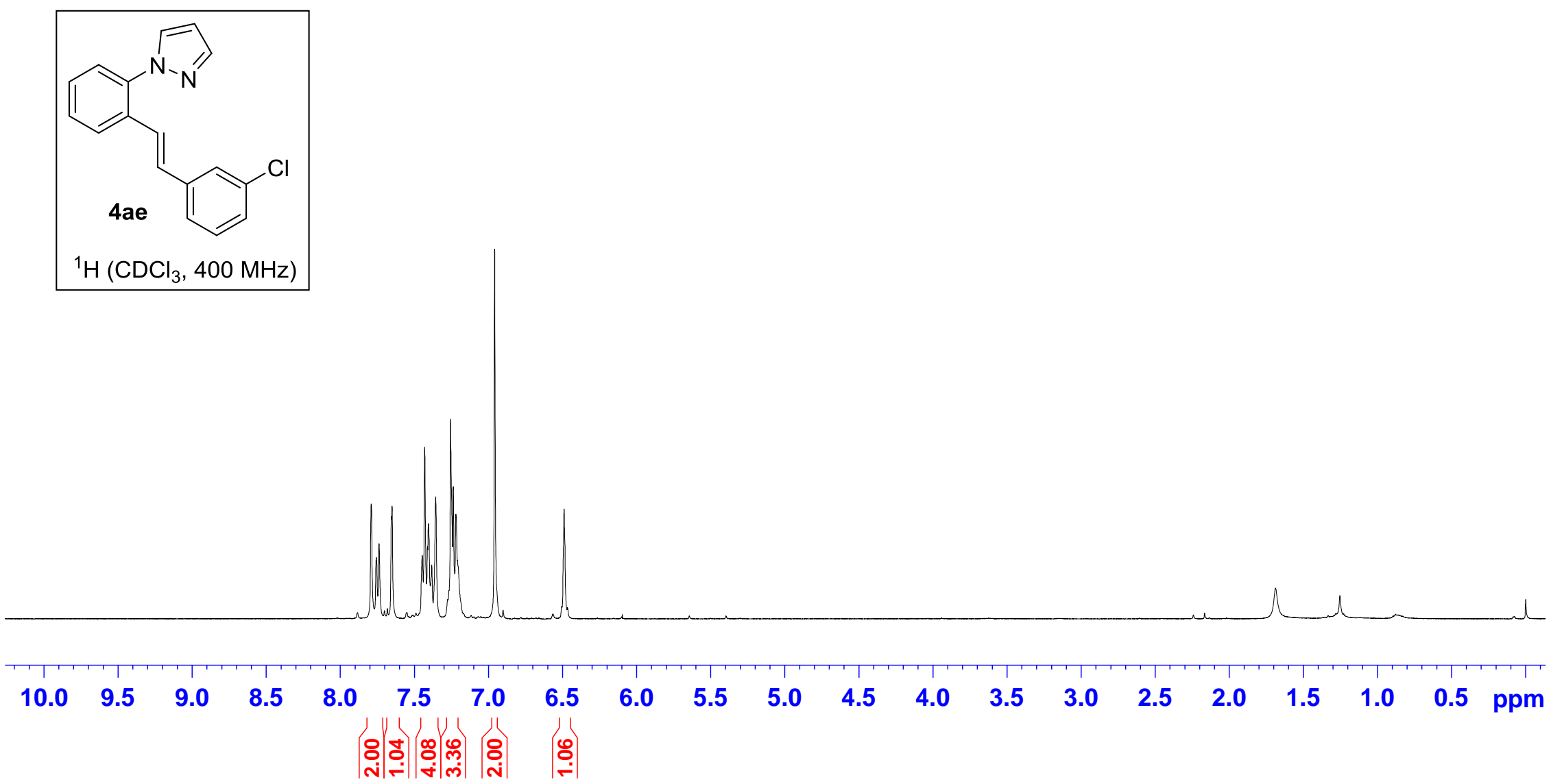




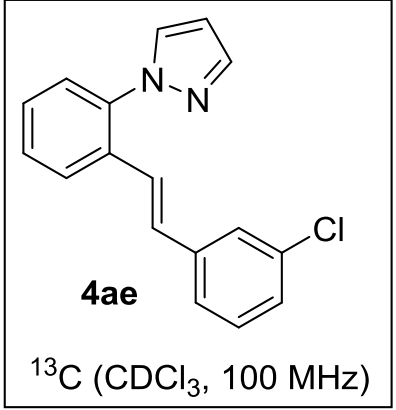



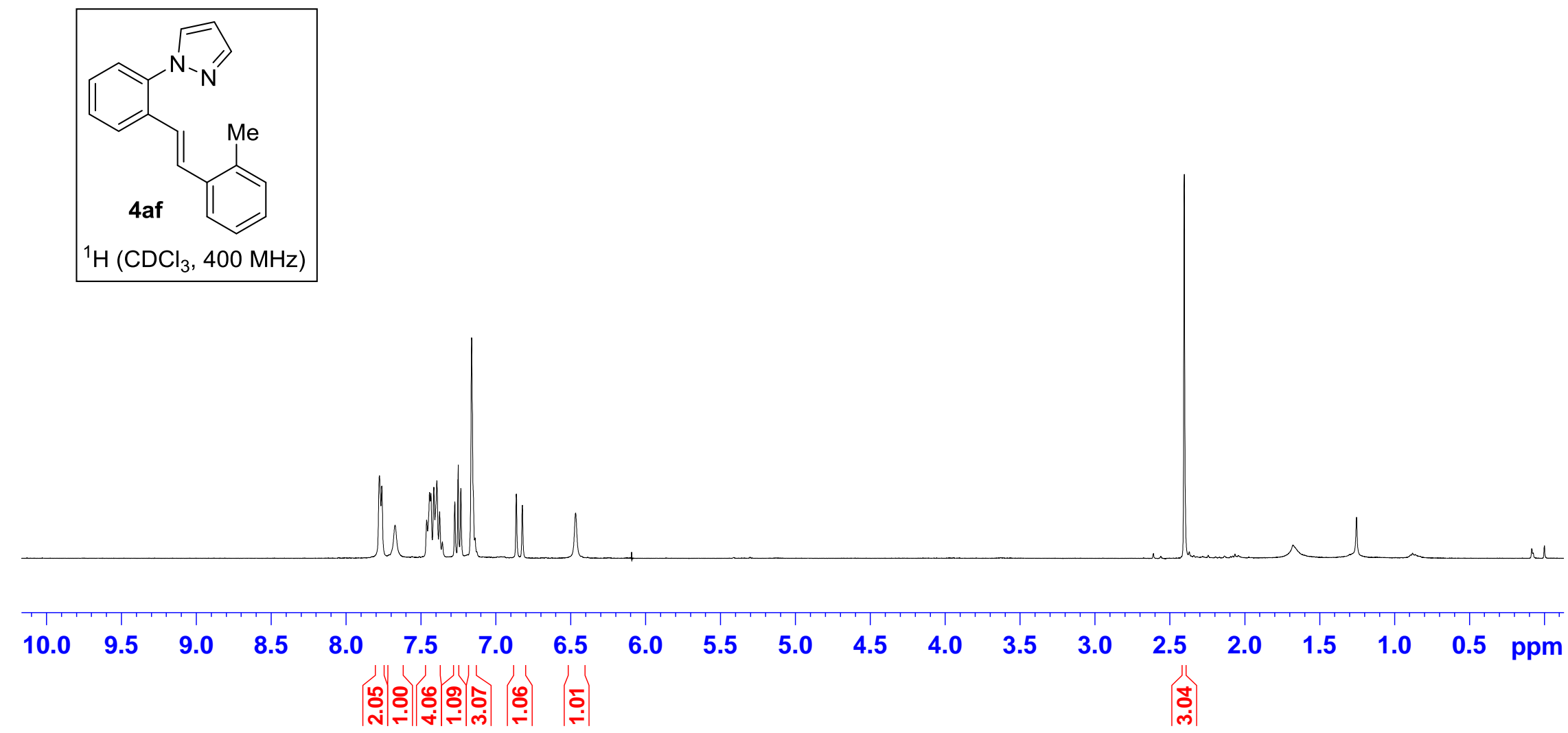


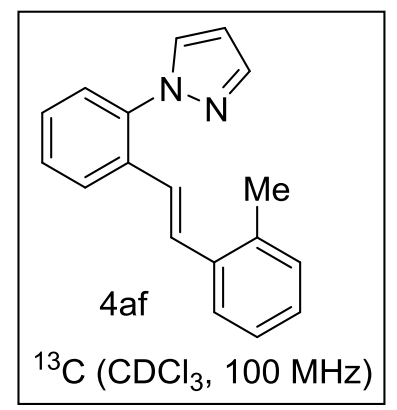




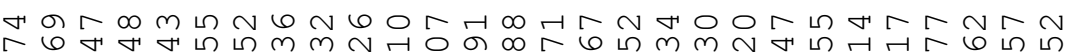

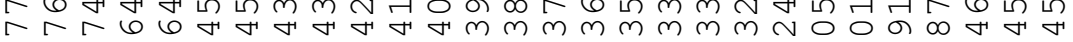

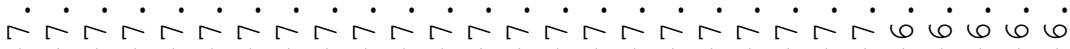
$\longrightarrow$
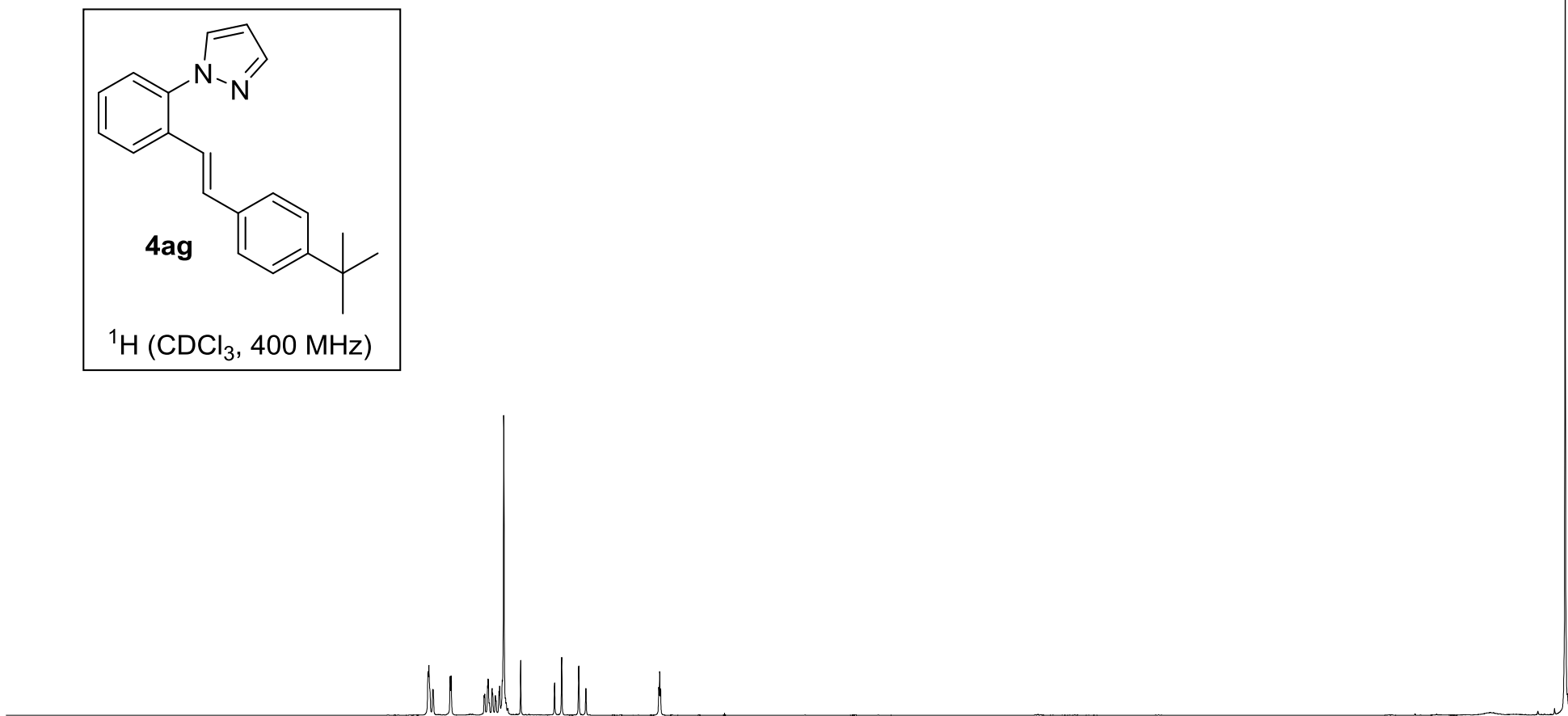

\subsection{8 .0

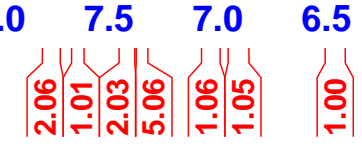

4.54 .0

3.5

\subsection{2 .5}

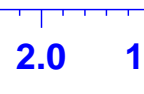

$\begin{array}{llll} & 1.0 & 0.5 & \text { ppm }\end{array}$ 


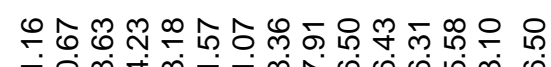

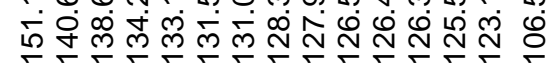

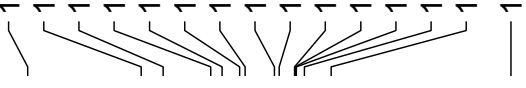

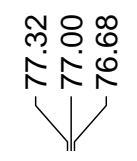

$\bar{్}$ ำ

ले ले
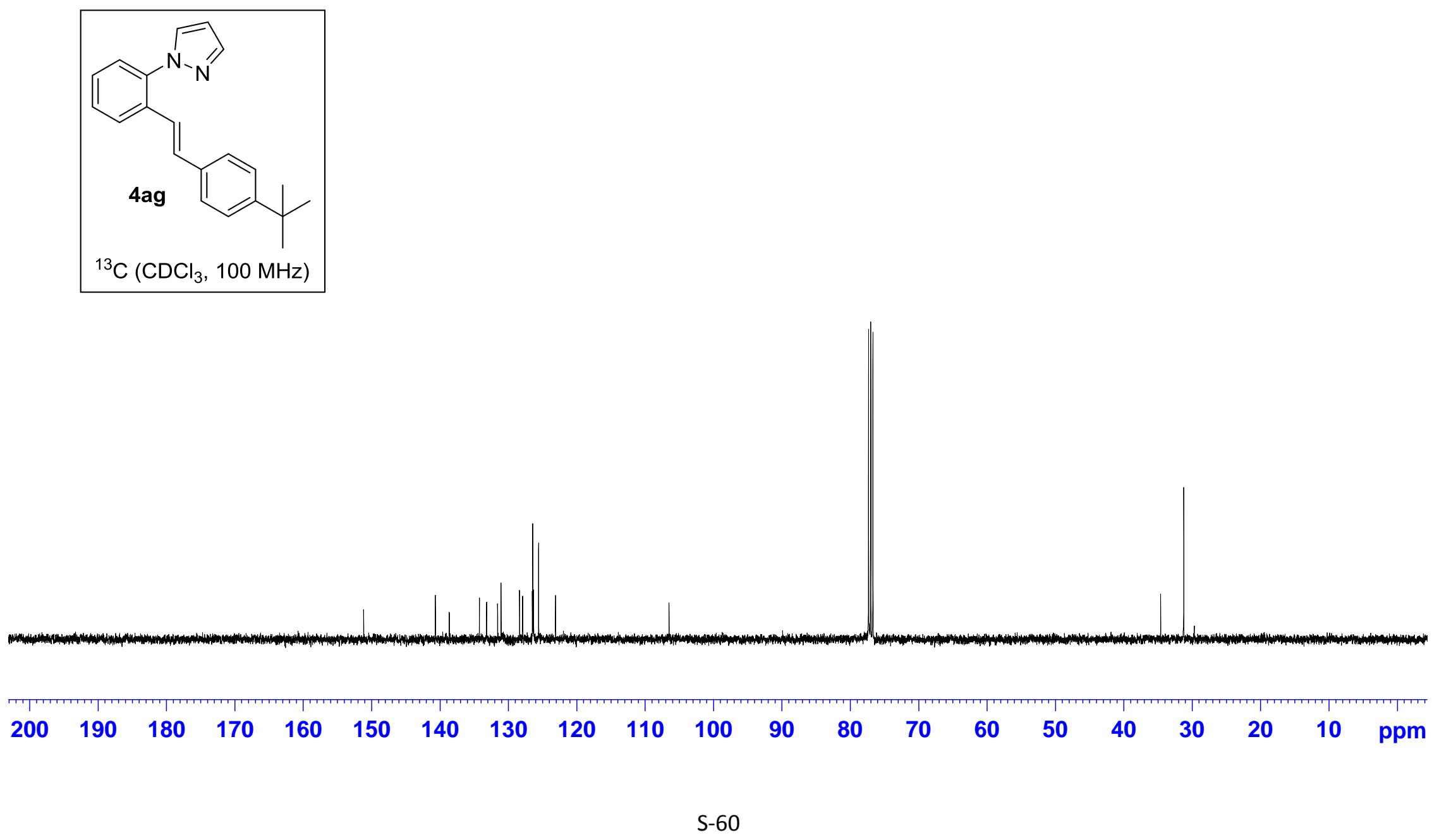


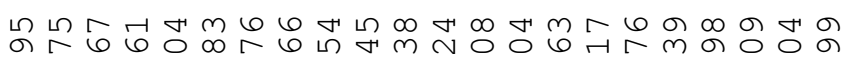

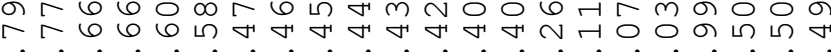

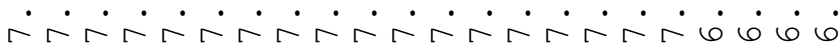

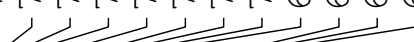
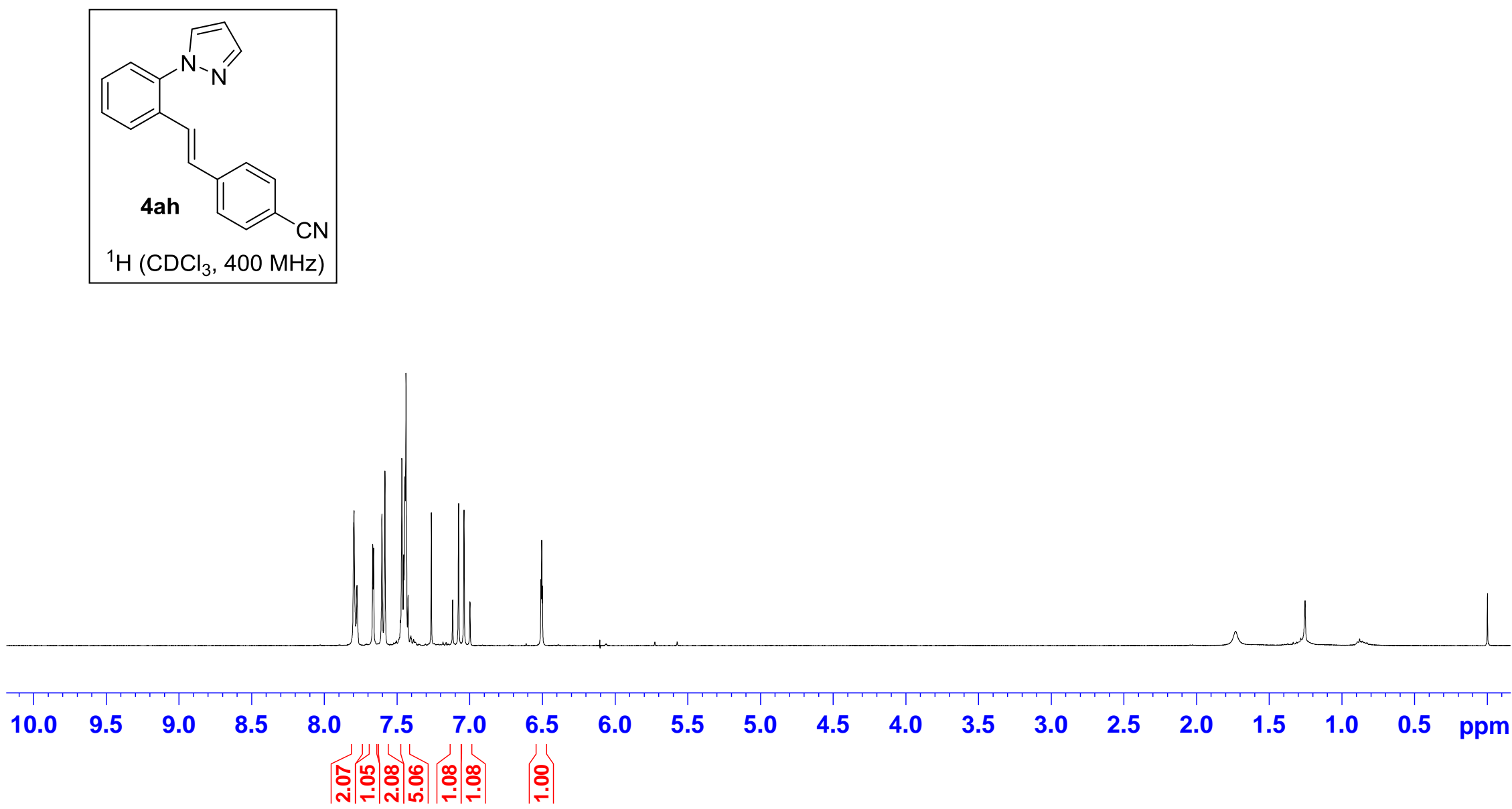

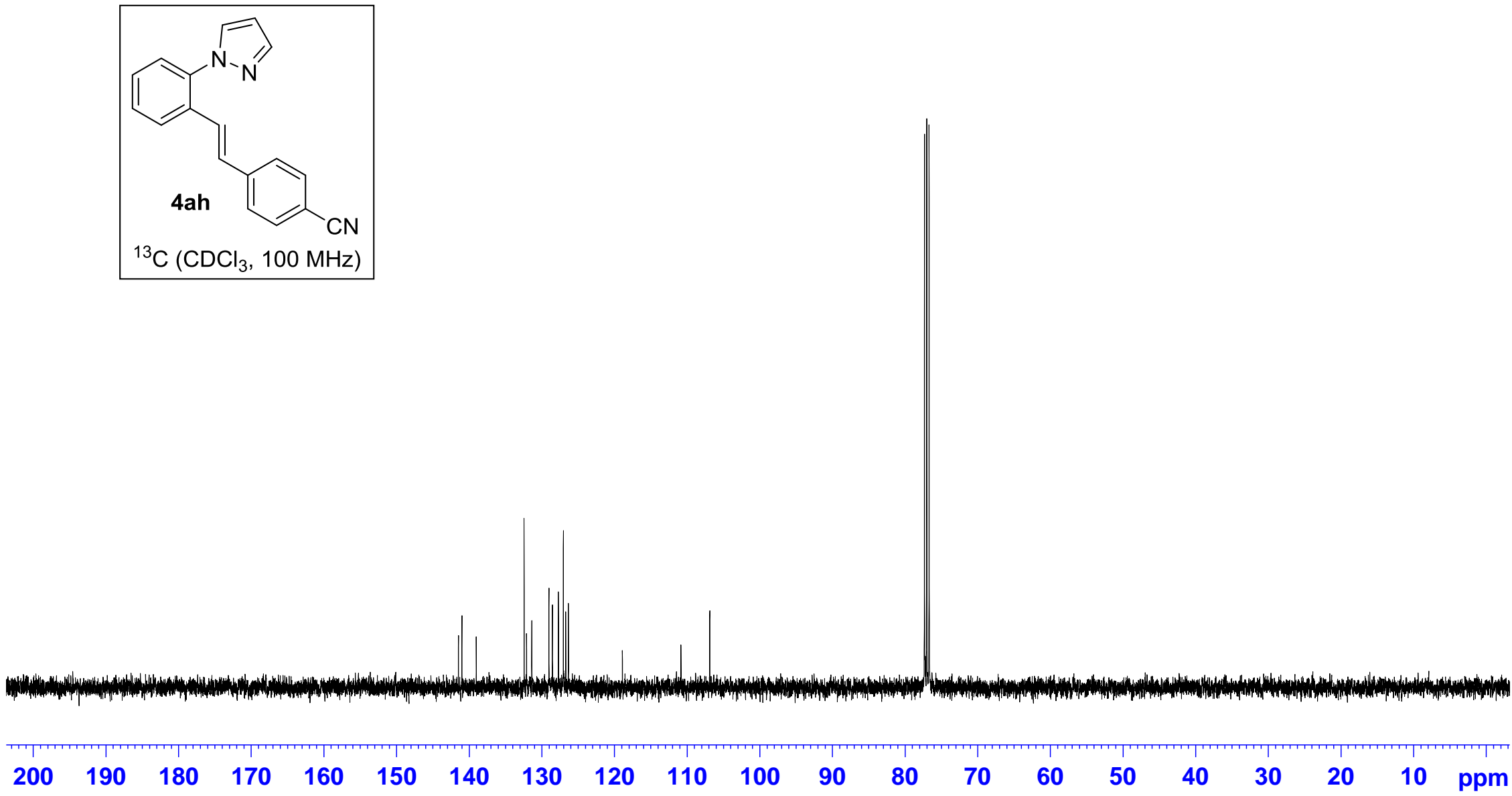

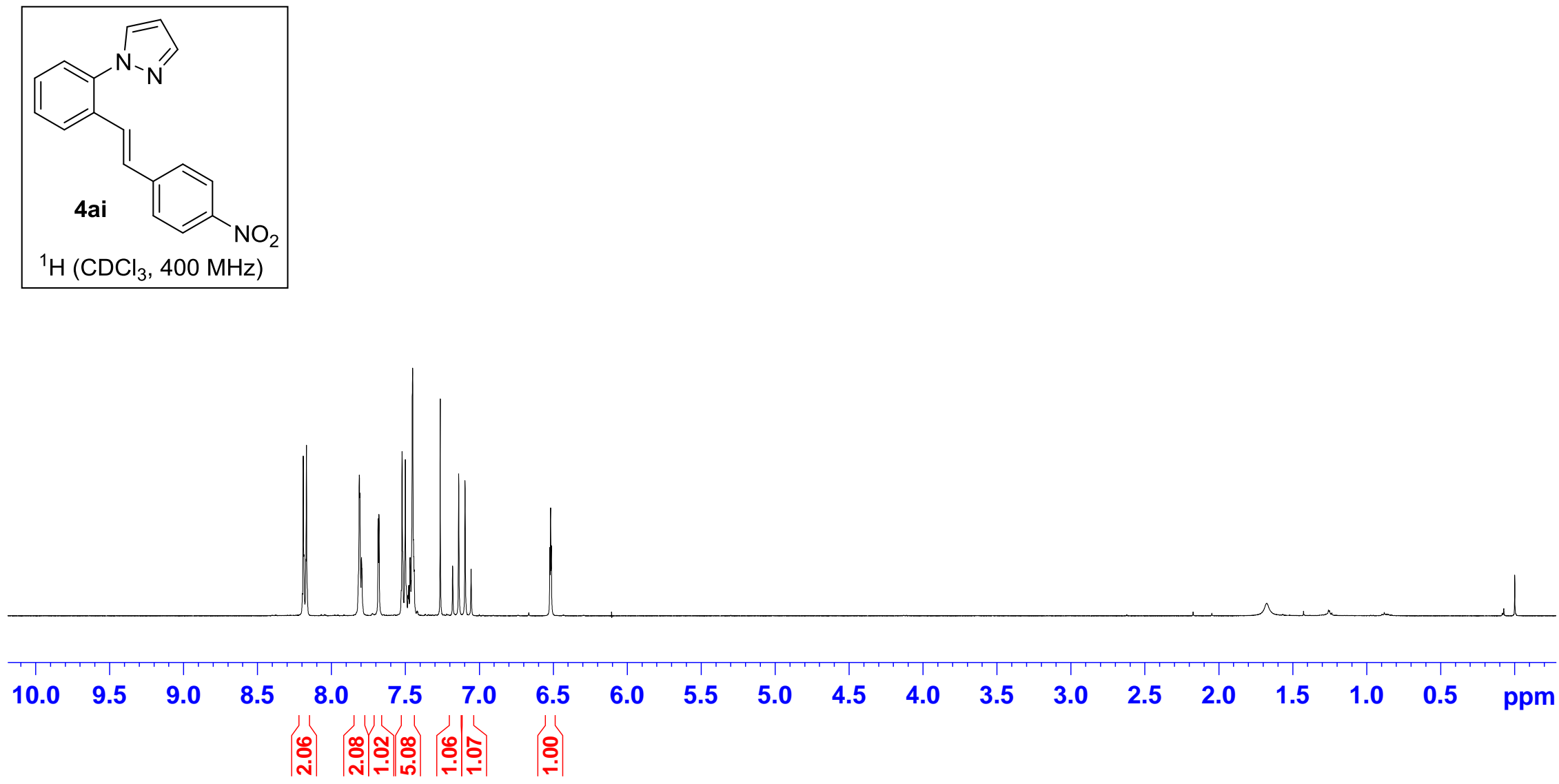

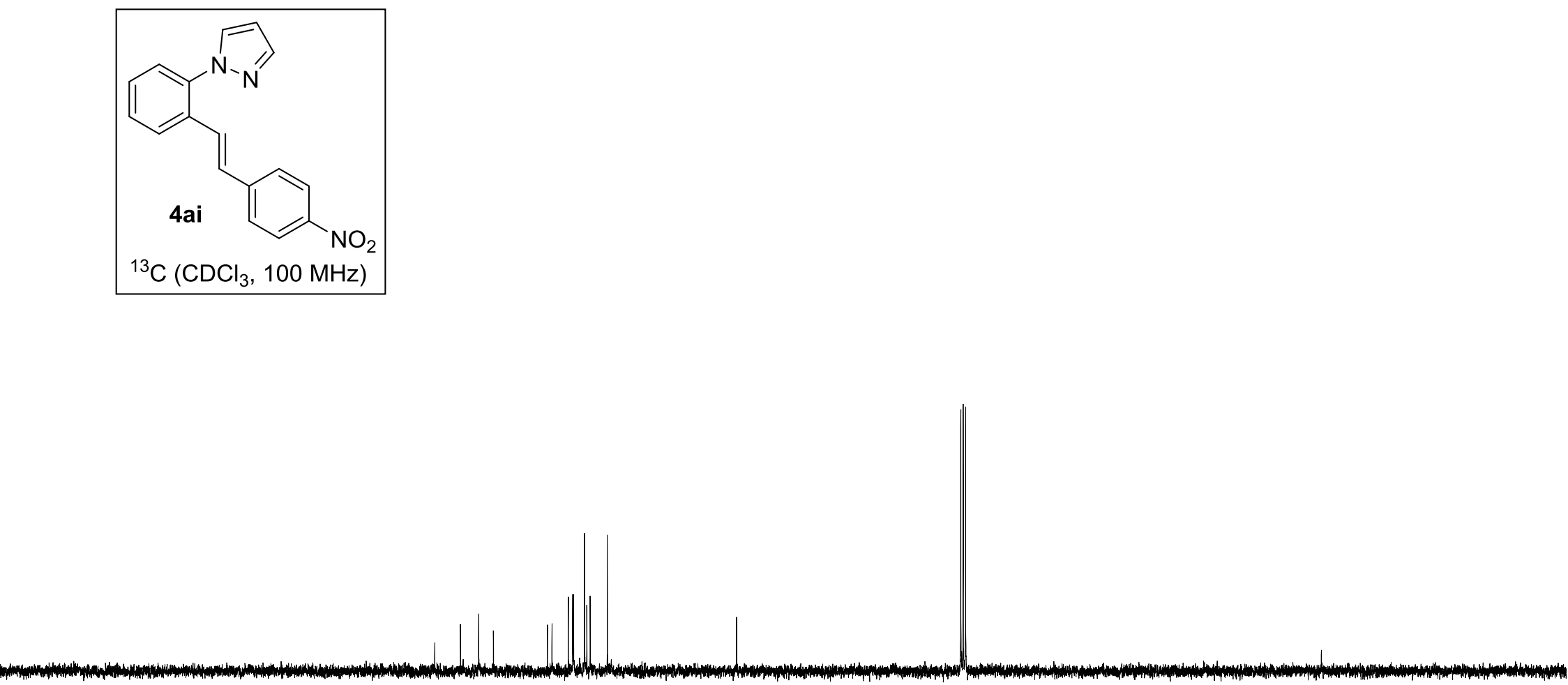

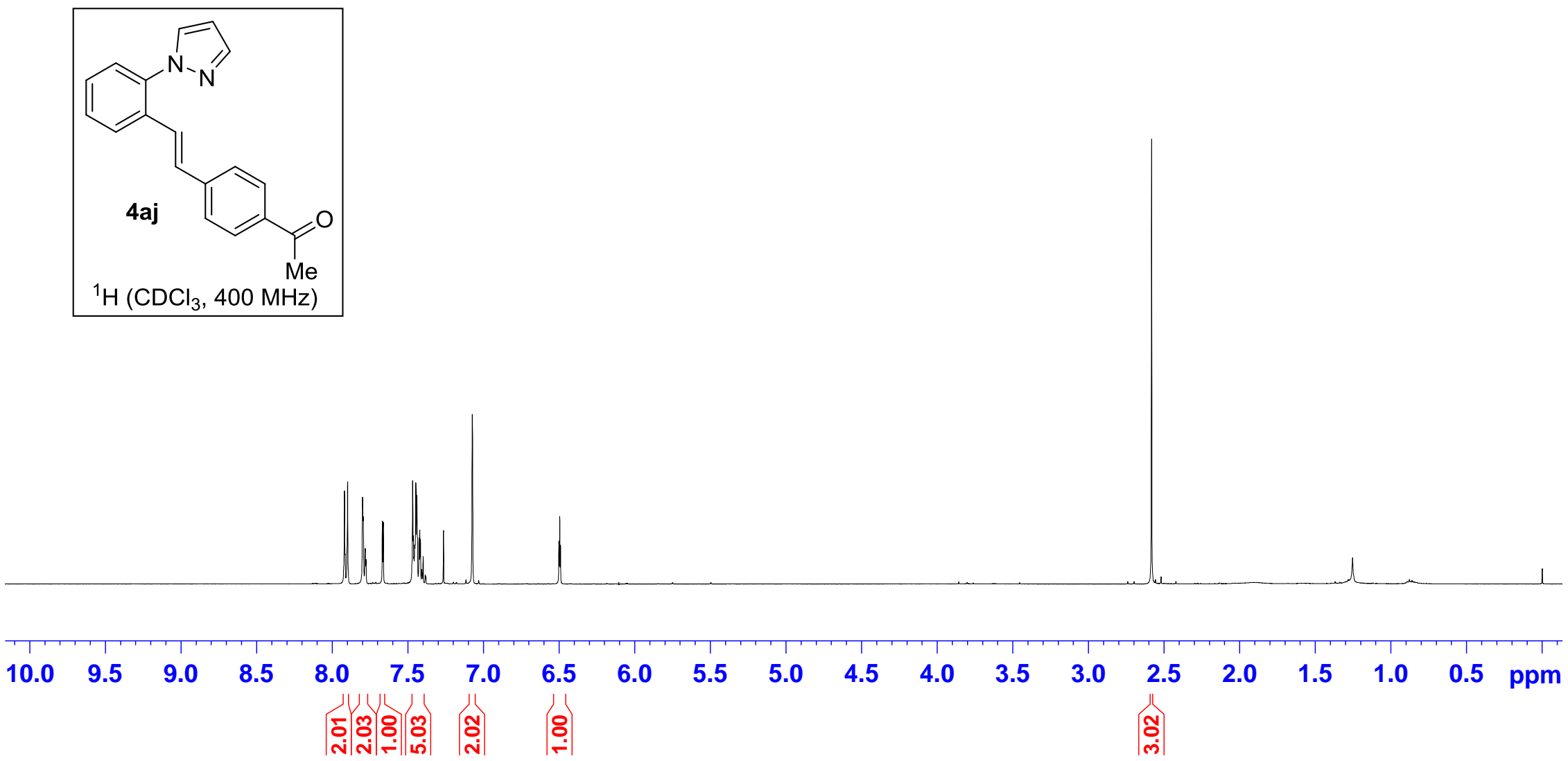

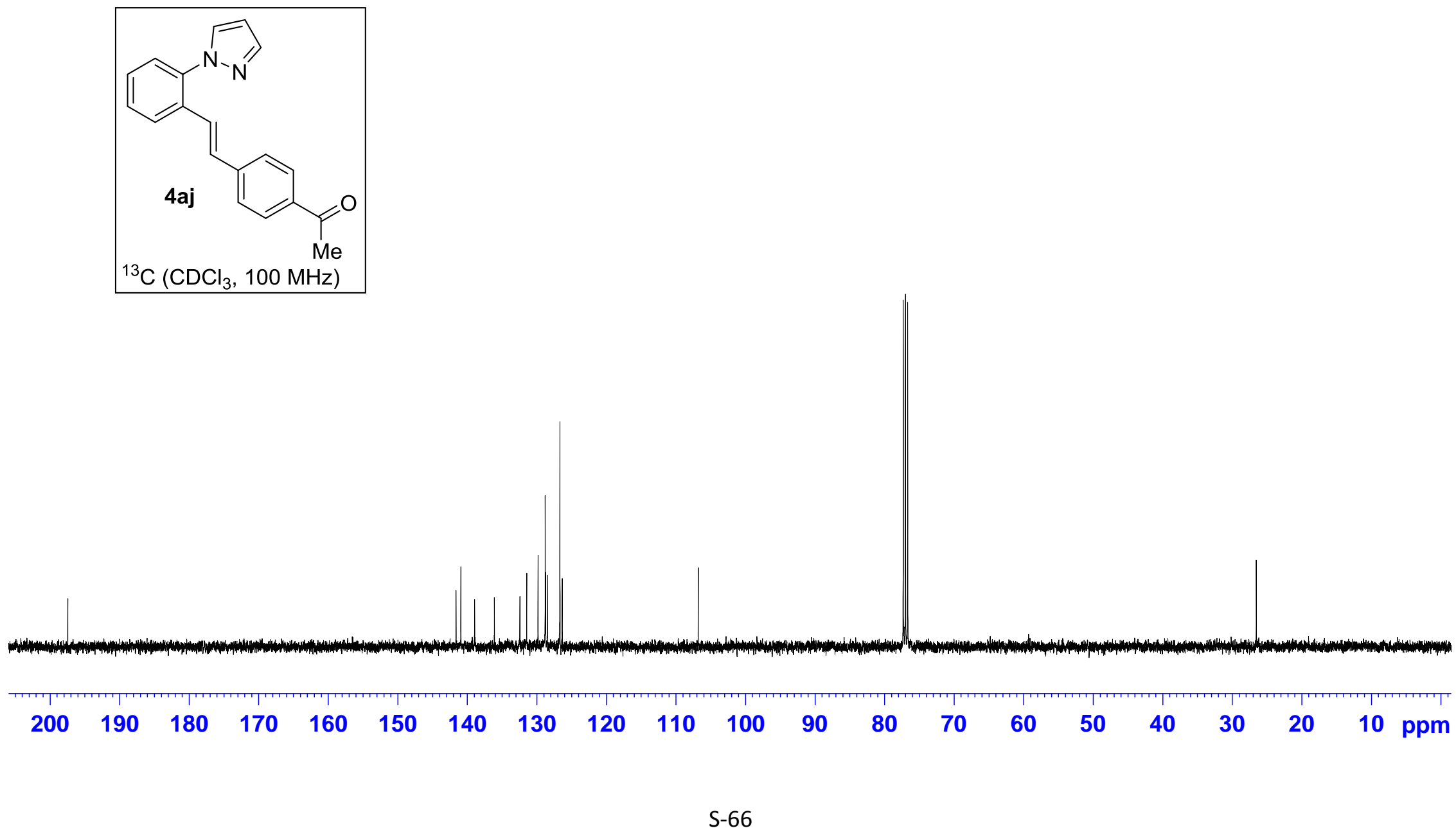

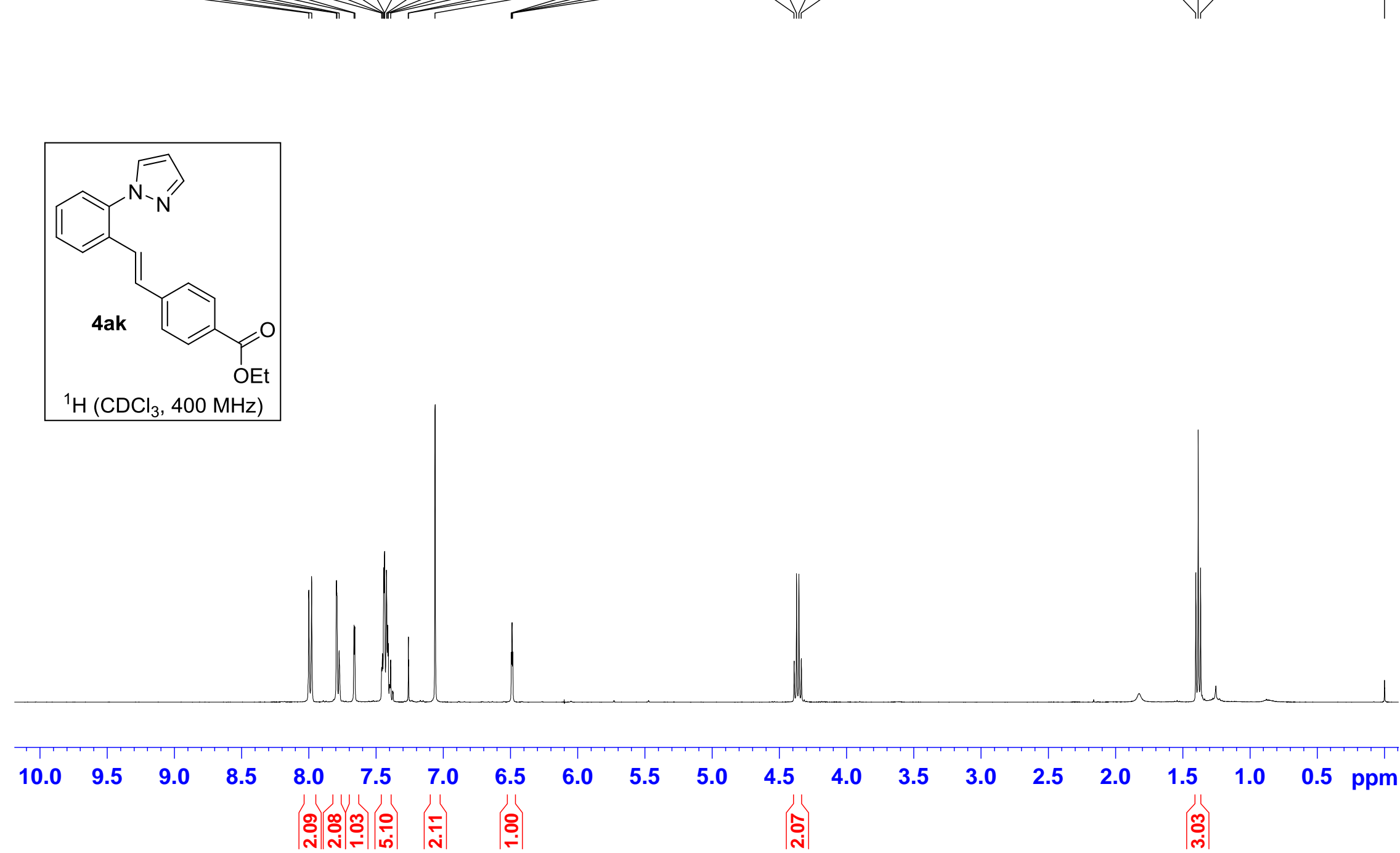


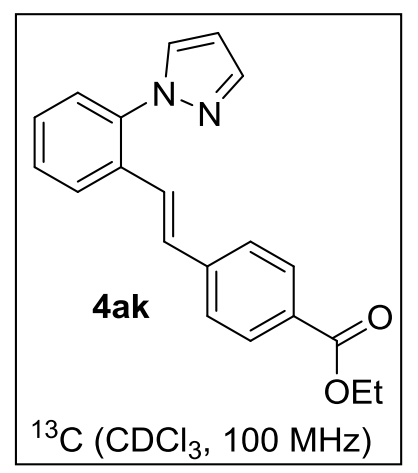

LIt 

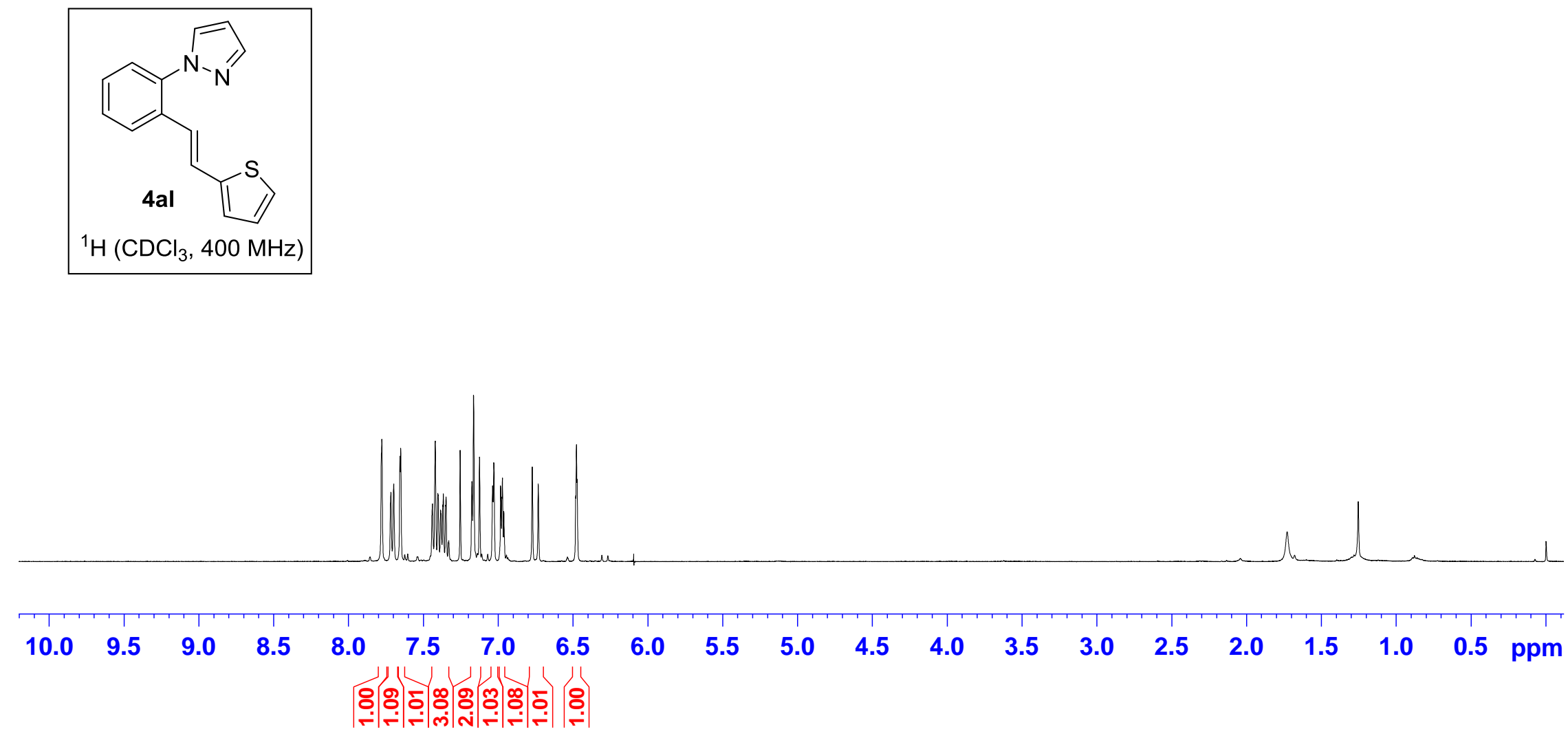


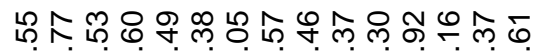

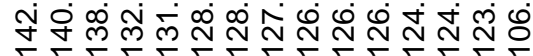

in

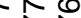

I/

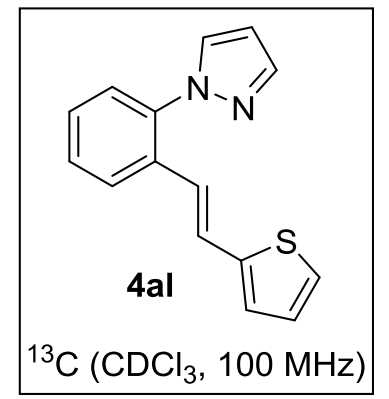

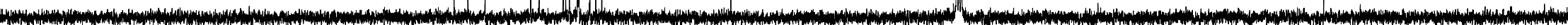
200
190
180
170
160
150
140
$130 \quad 120$
12011
110
100
90
80
70
$60 \quad 50$
$50 \quad 40$
30
20
10 ppm 
m $m$ m

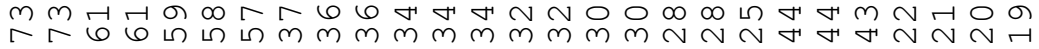

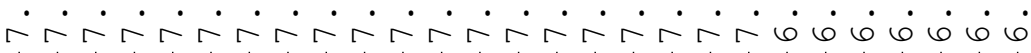

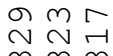

$\dot{-1} \dot{-} \dot{ }$

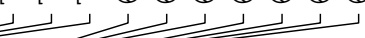
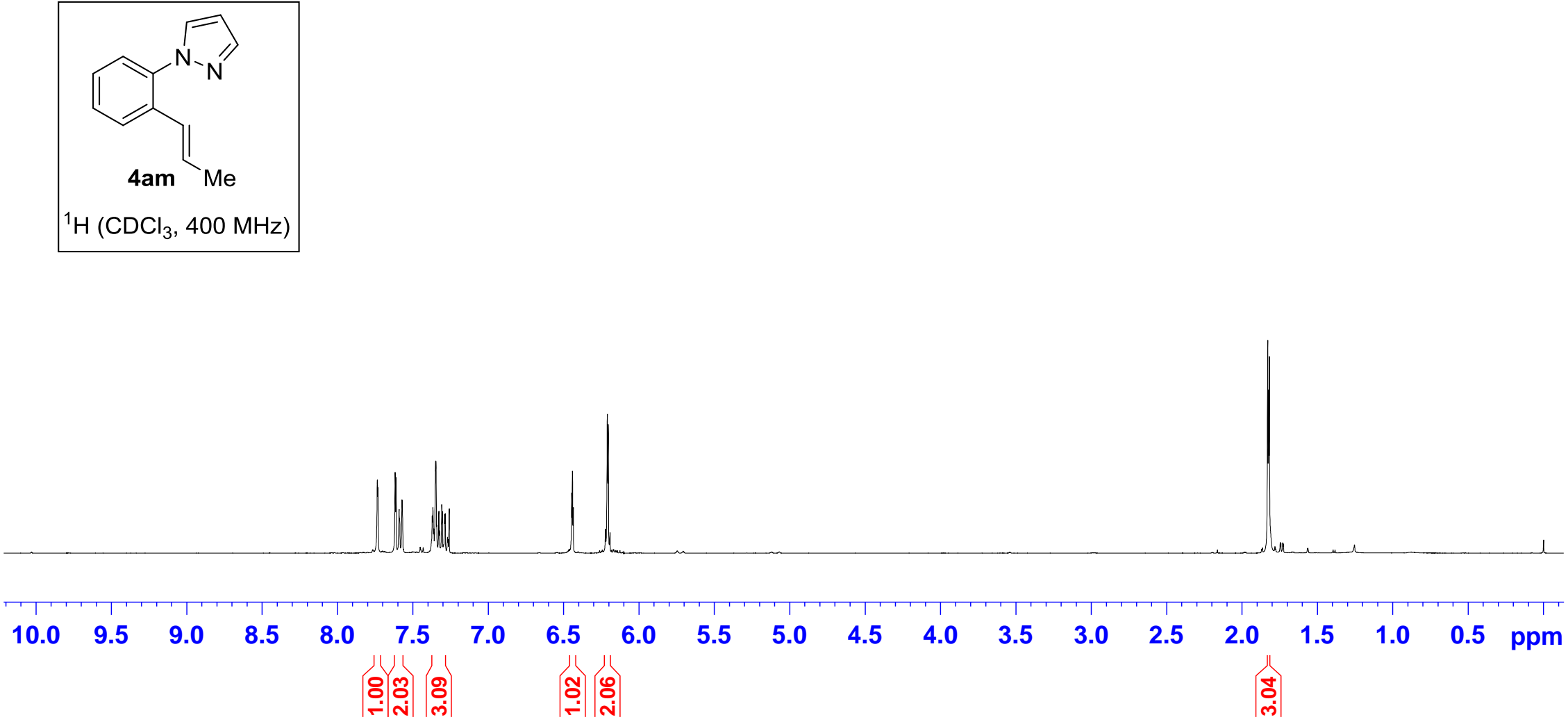

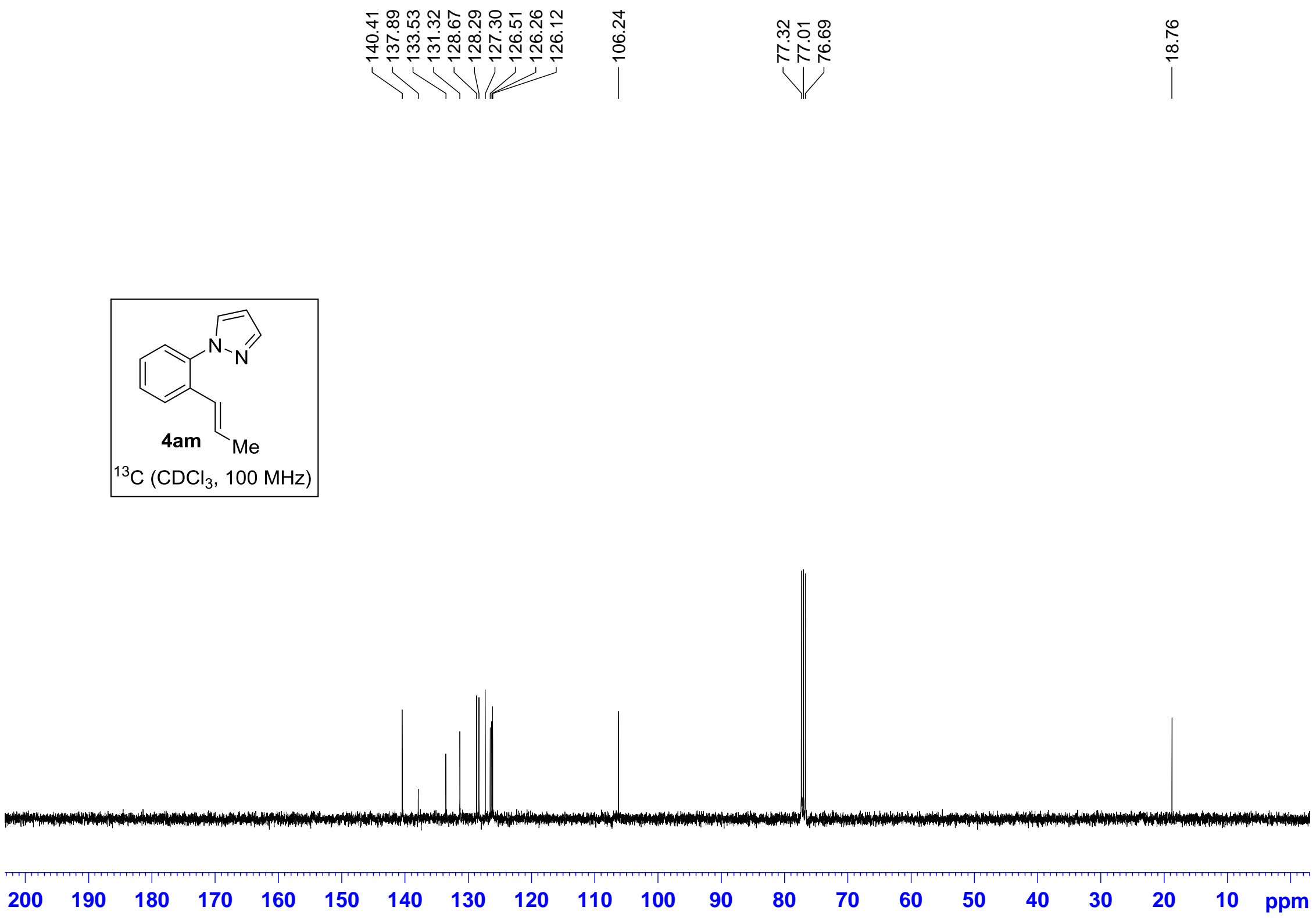

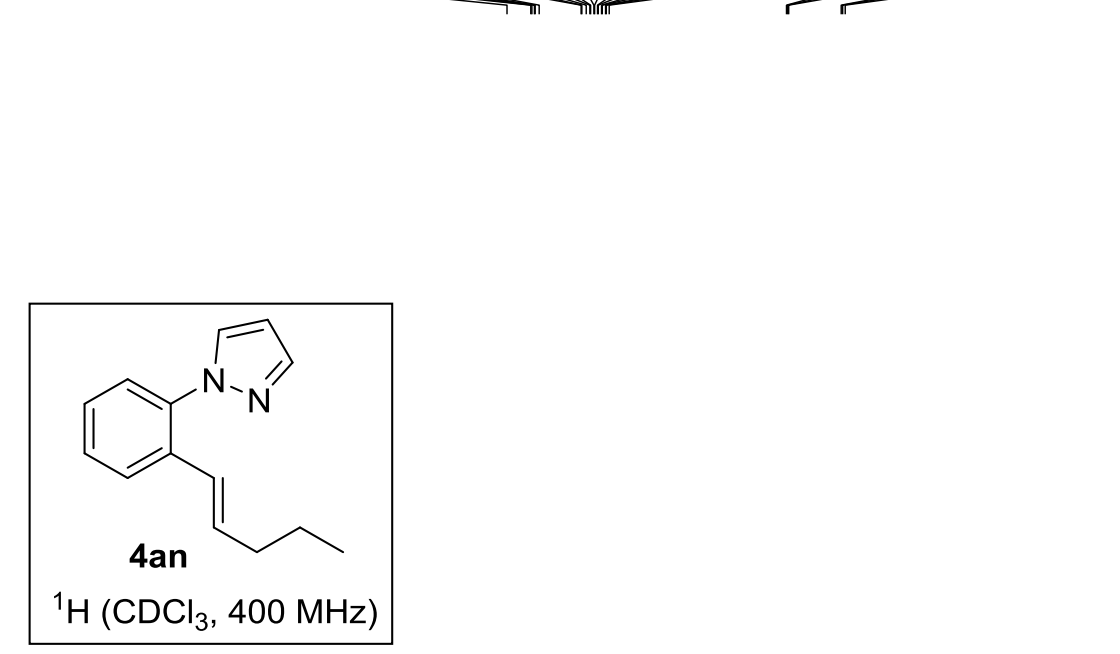


$\underbrace{\infty}_{\mid=1 / l}$

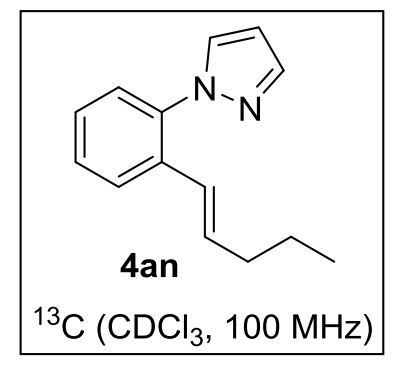

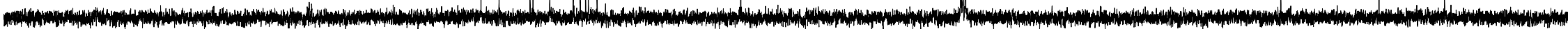

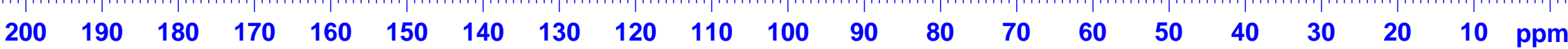




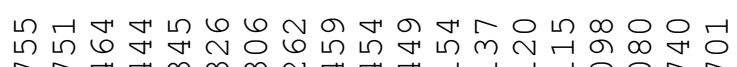

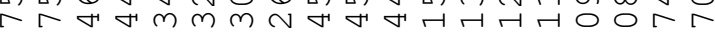

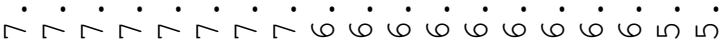

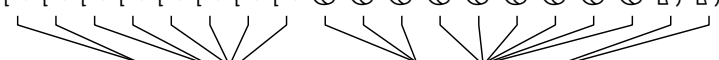

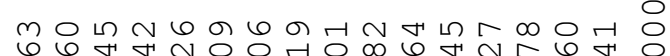

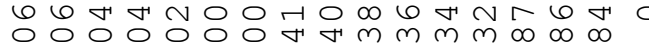
$\dot{\sim} \dot{\sim} \dot{\sim} \dot{\sim} \dot{\sim} \dot{r} \dot{r} \dot{r} \dot{r} \dot{i} \dot{0} 0 \dot{0}$ $3 \times 1 / 10$
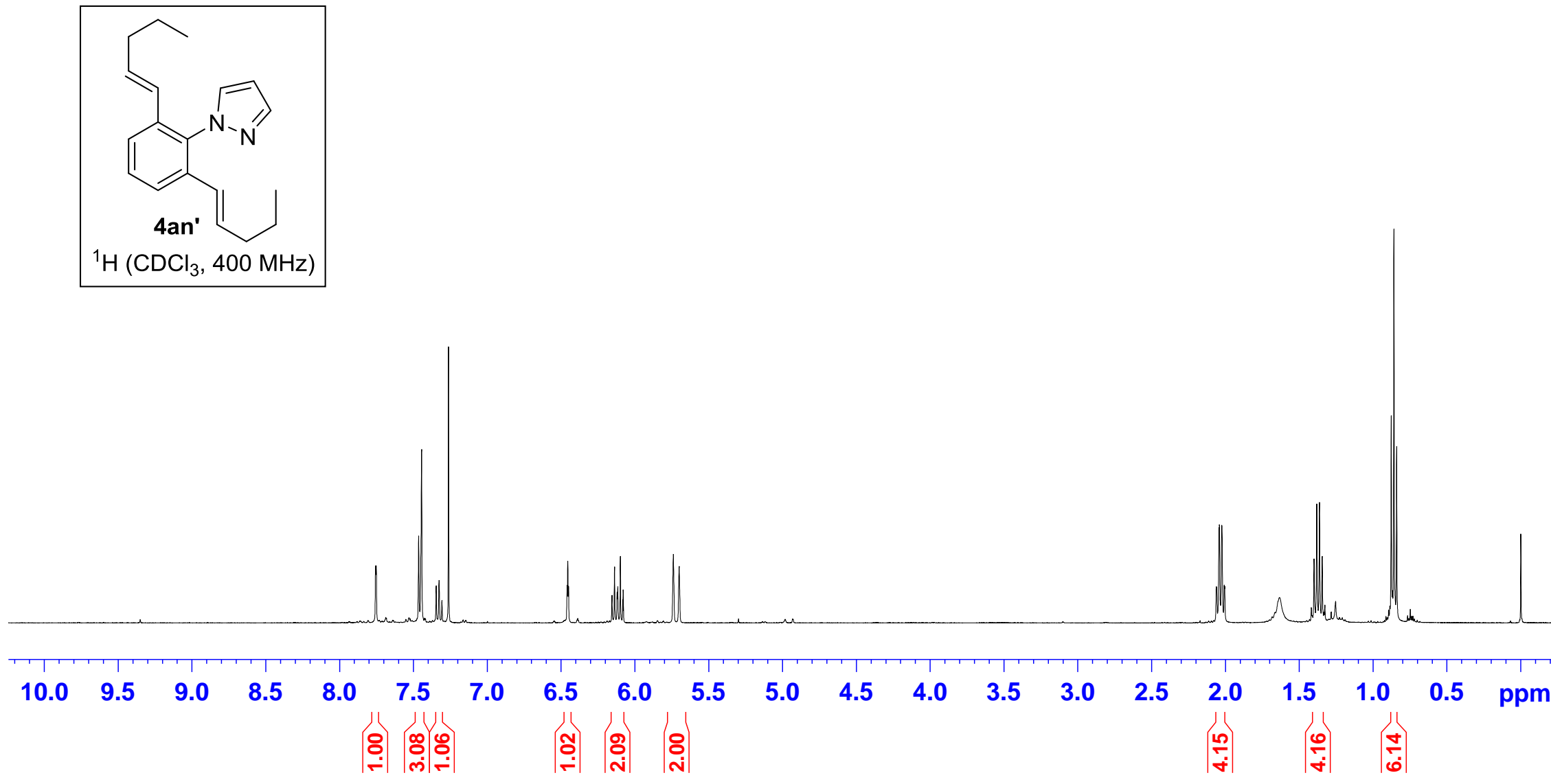


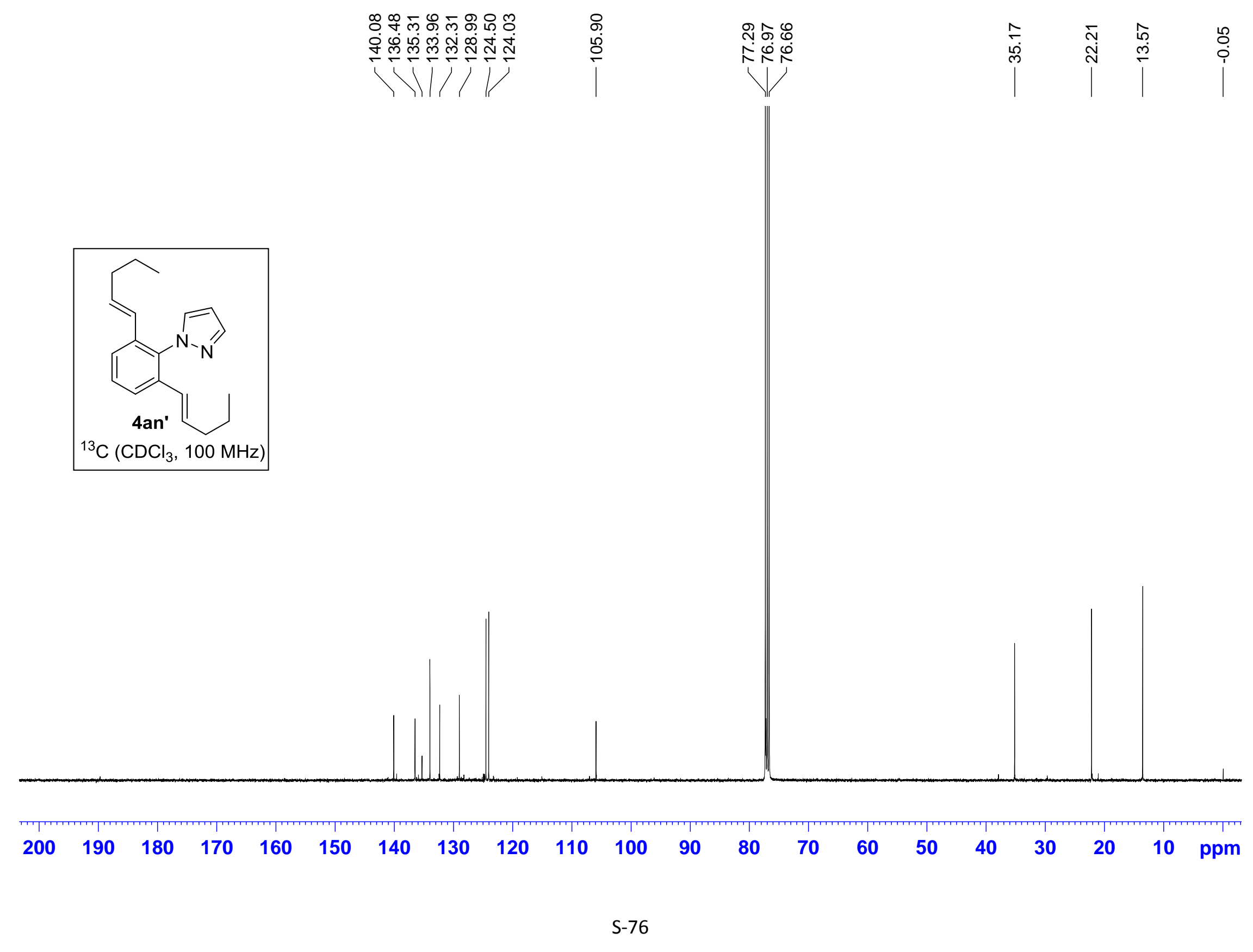




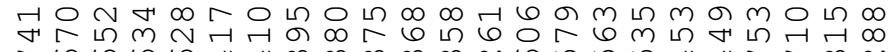

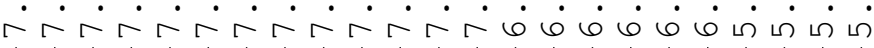

(n)
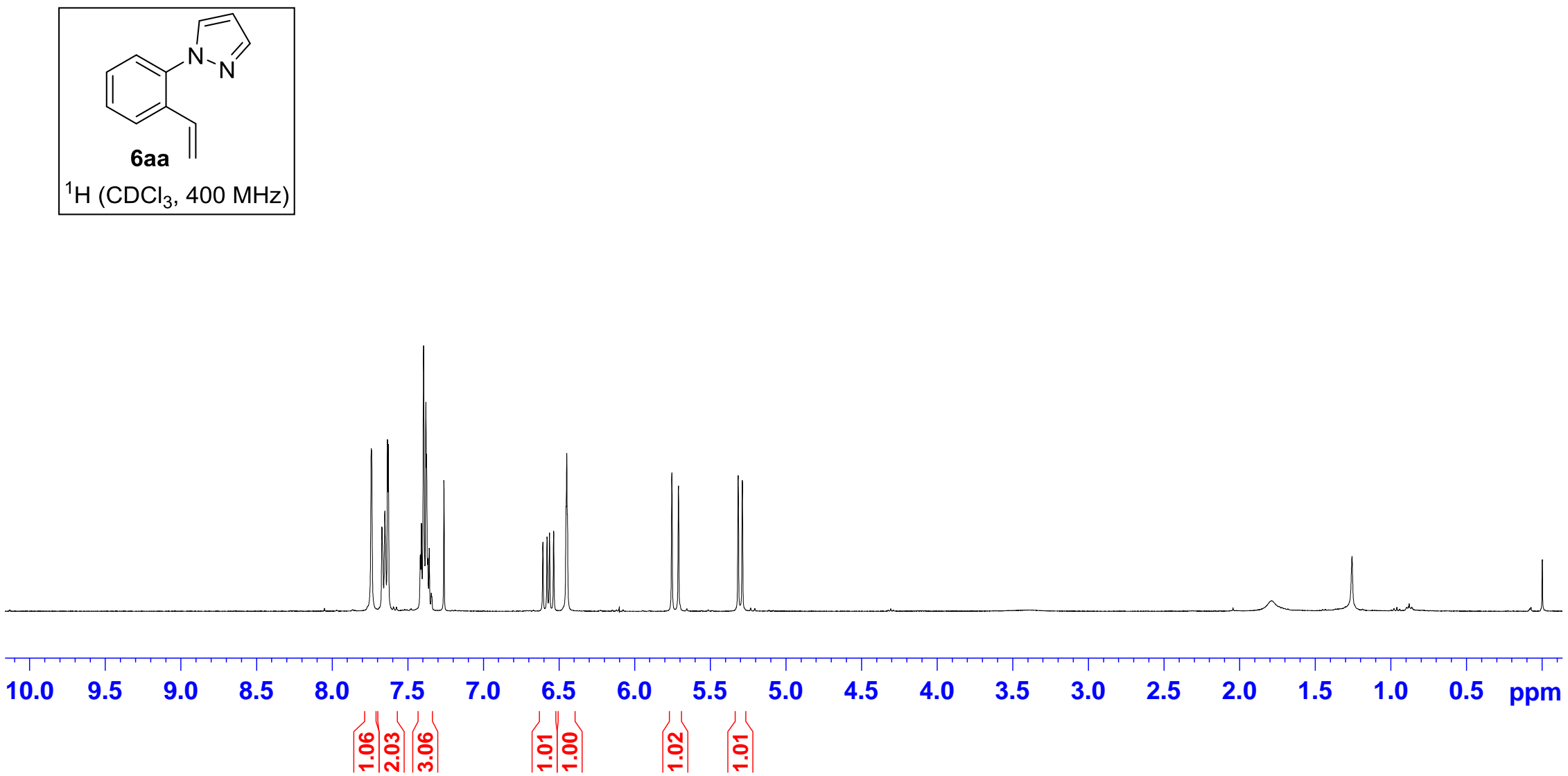

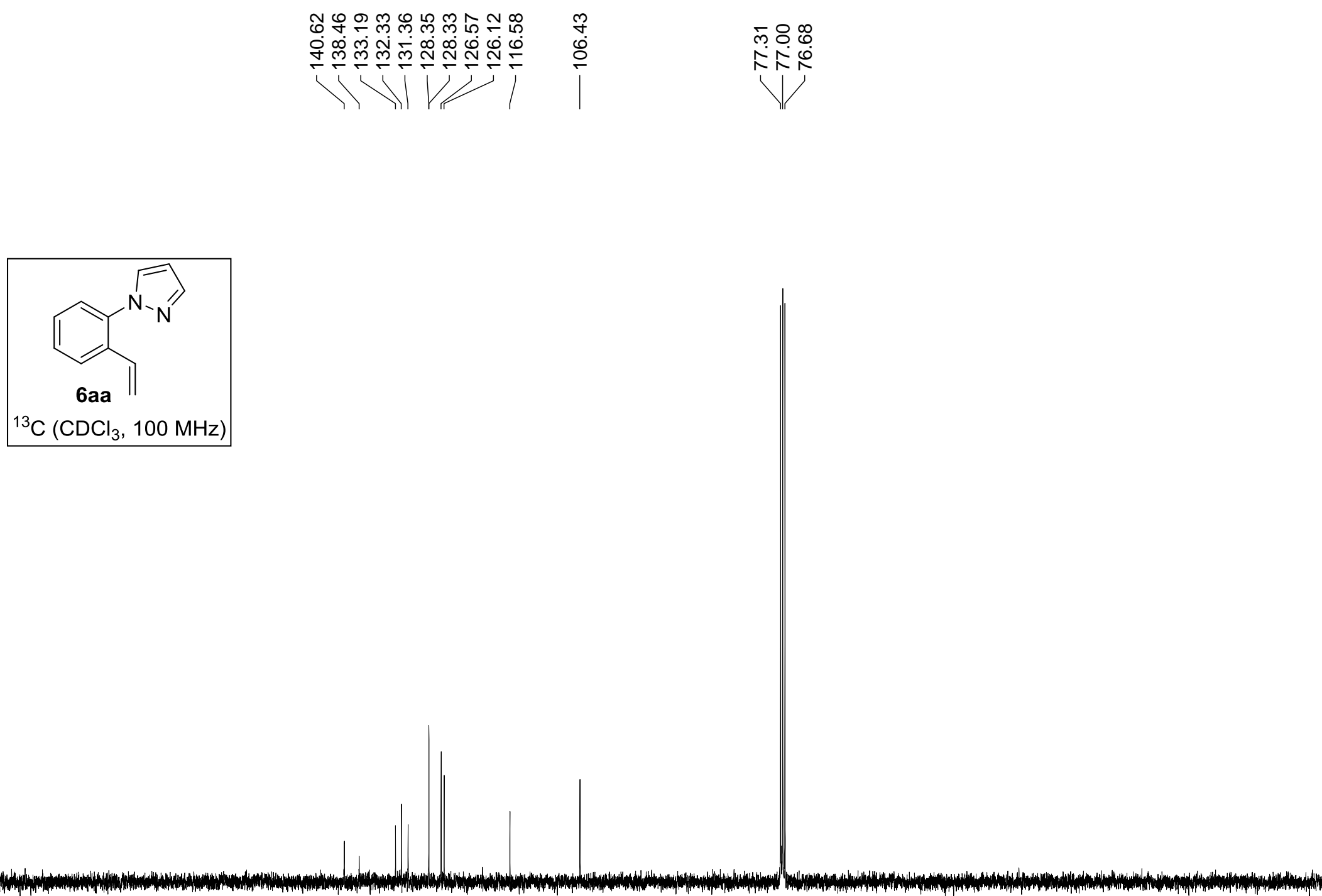

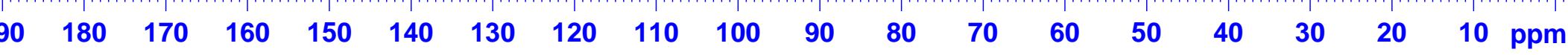




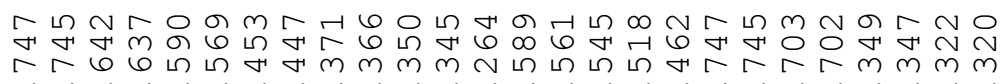

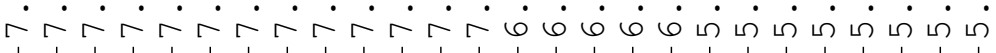

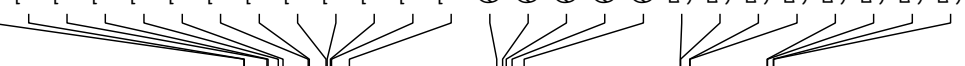
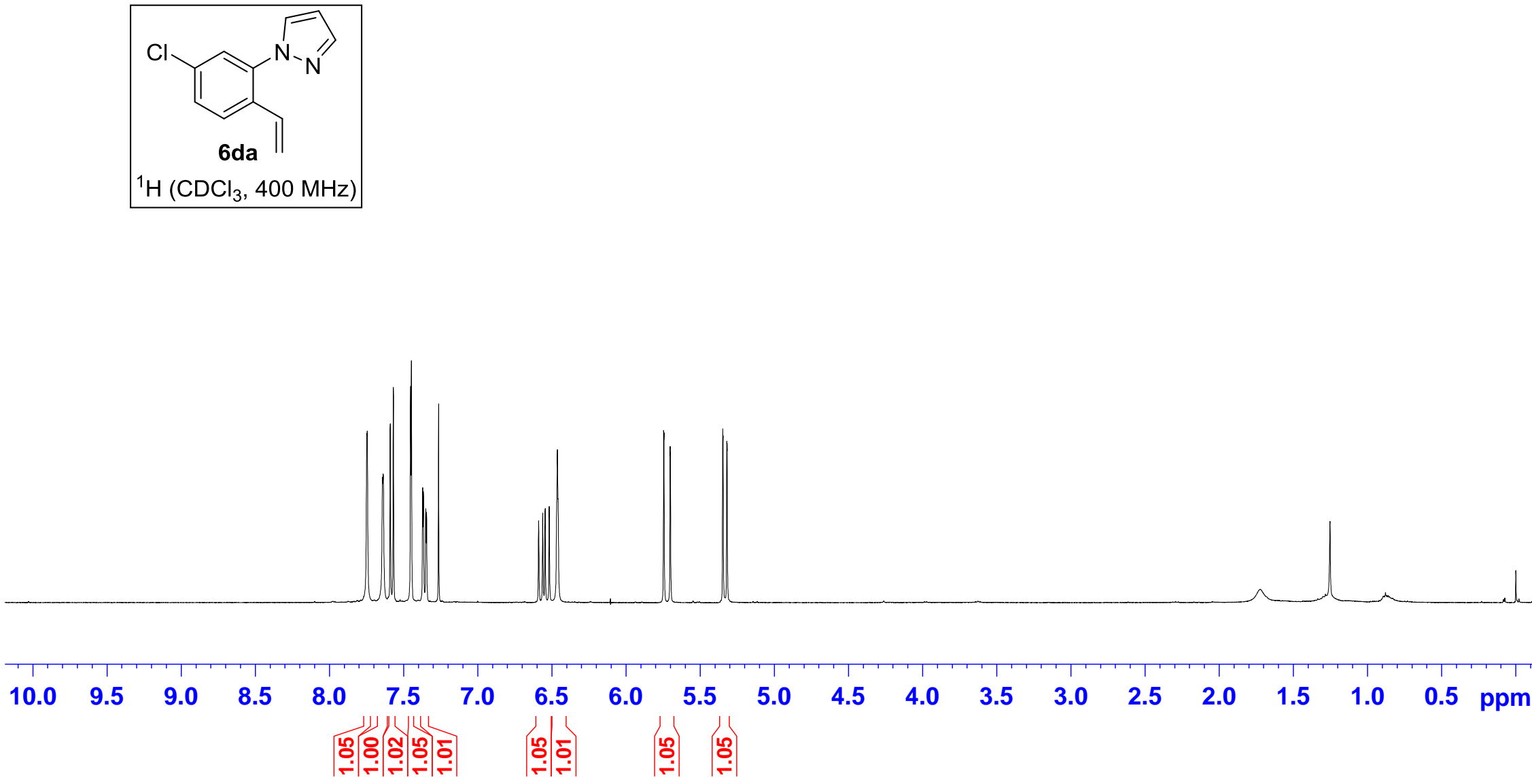

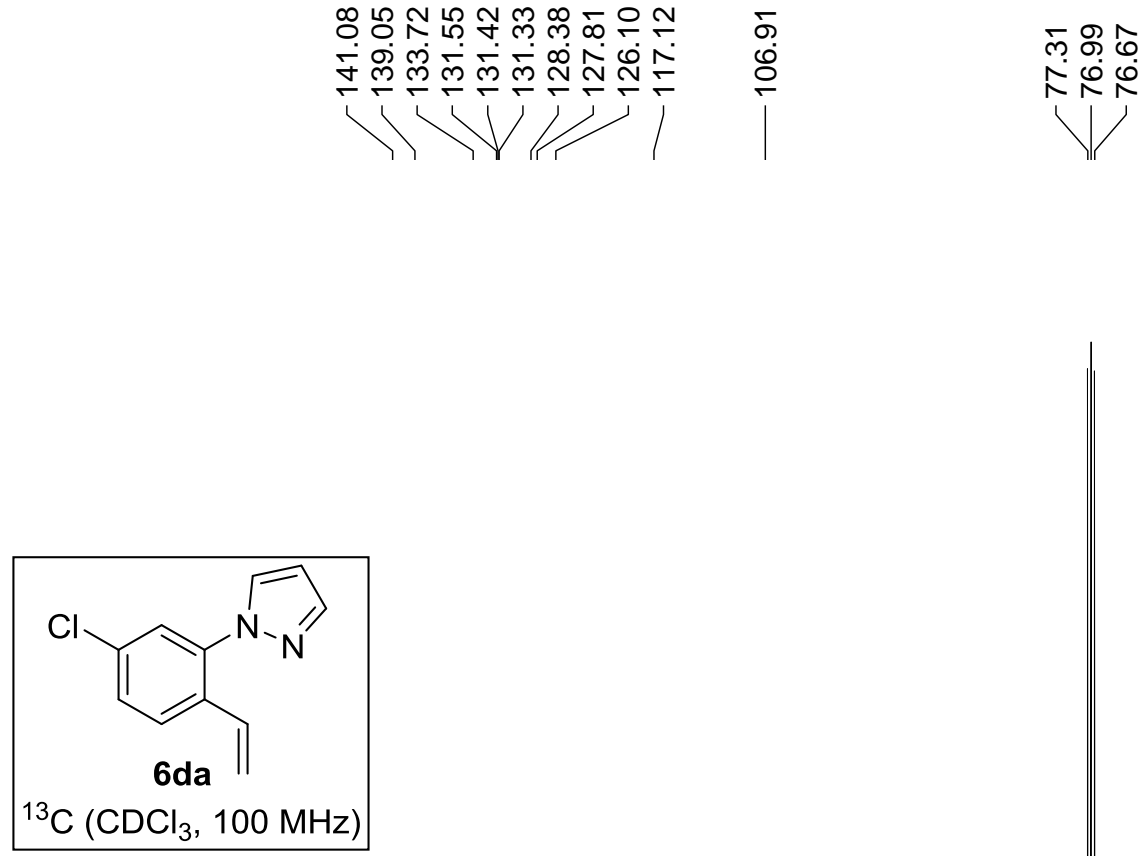

H.

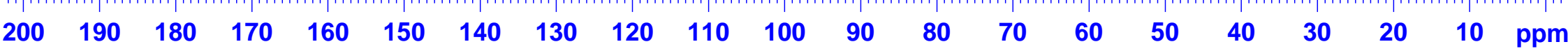




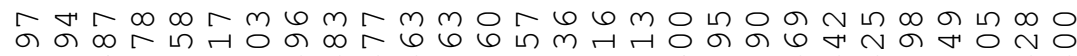

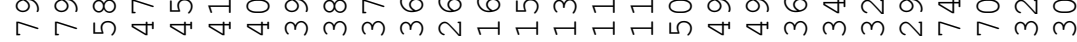

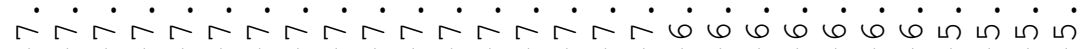
L
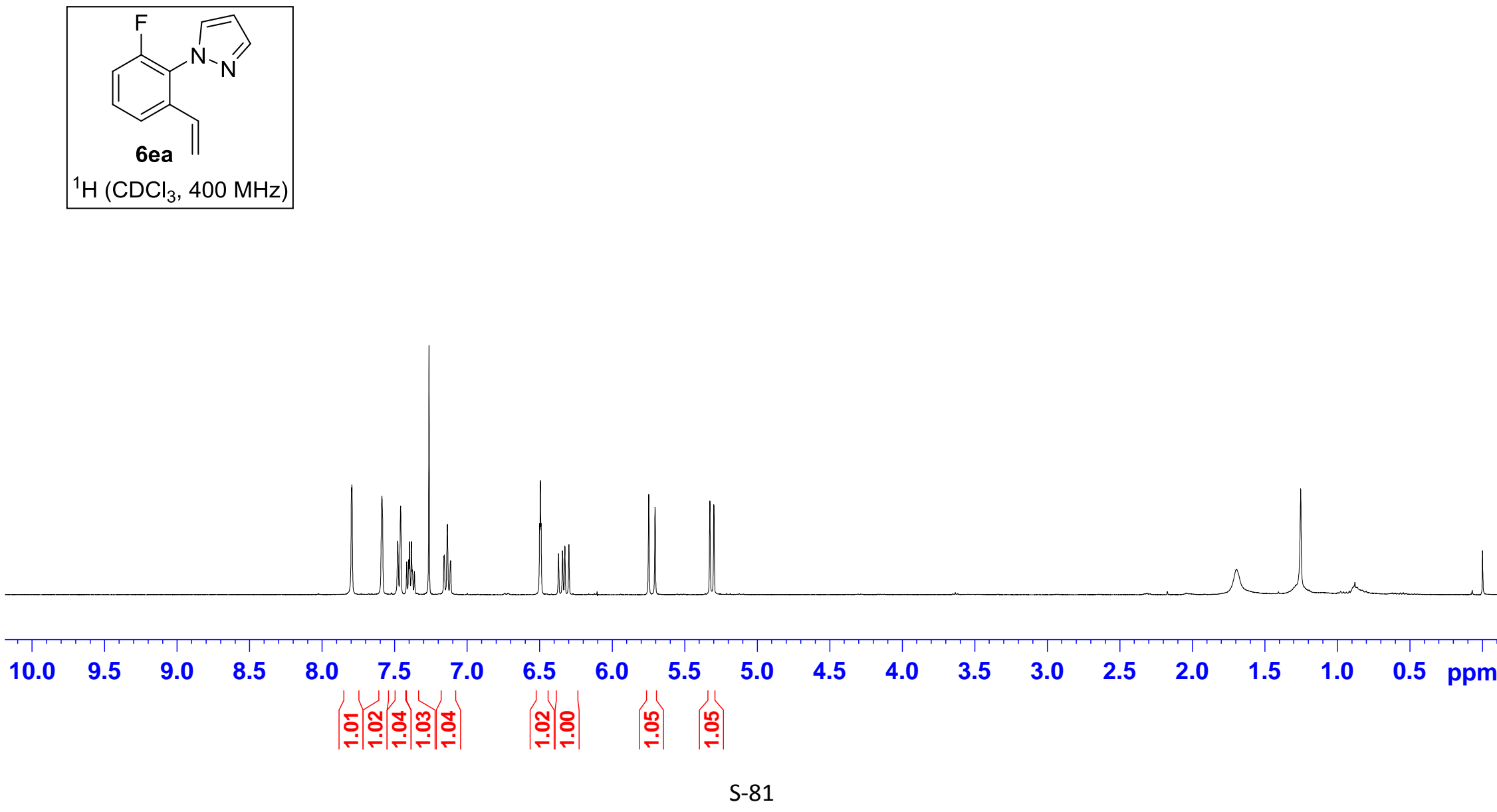


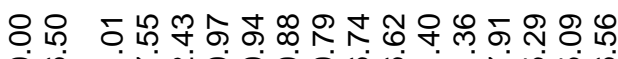

هั

लंखि

र下乐
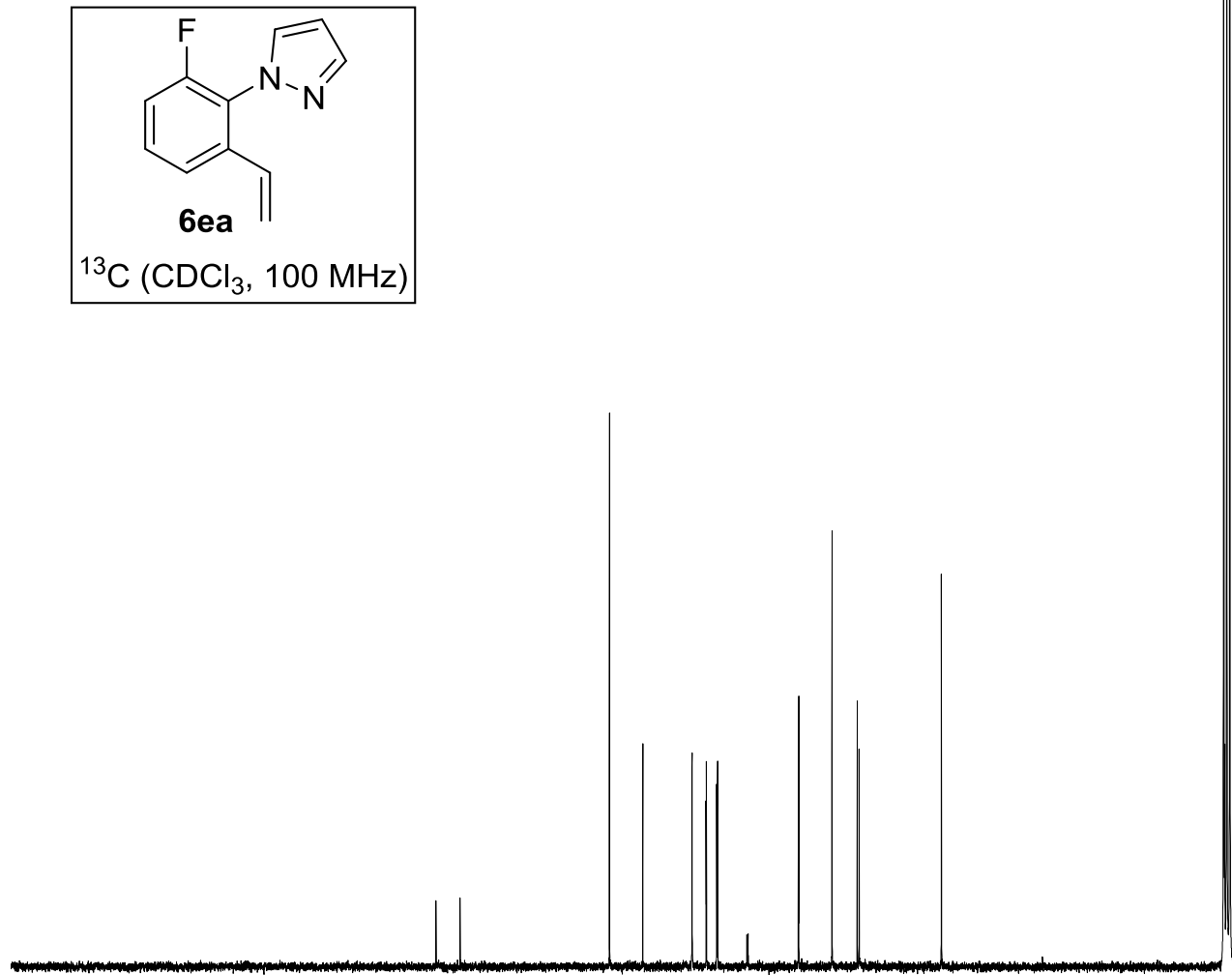


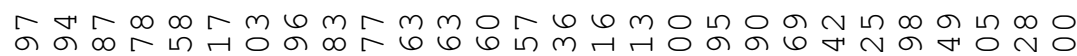

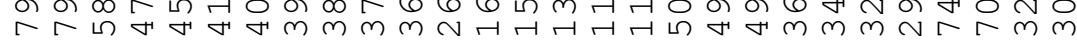

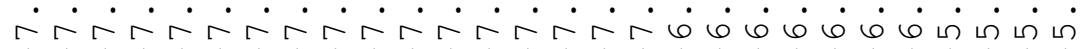
m
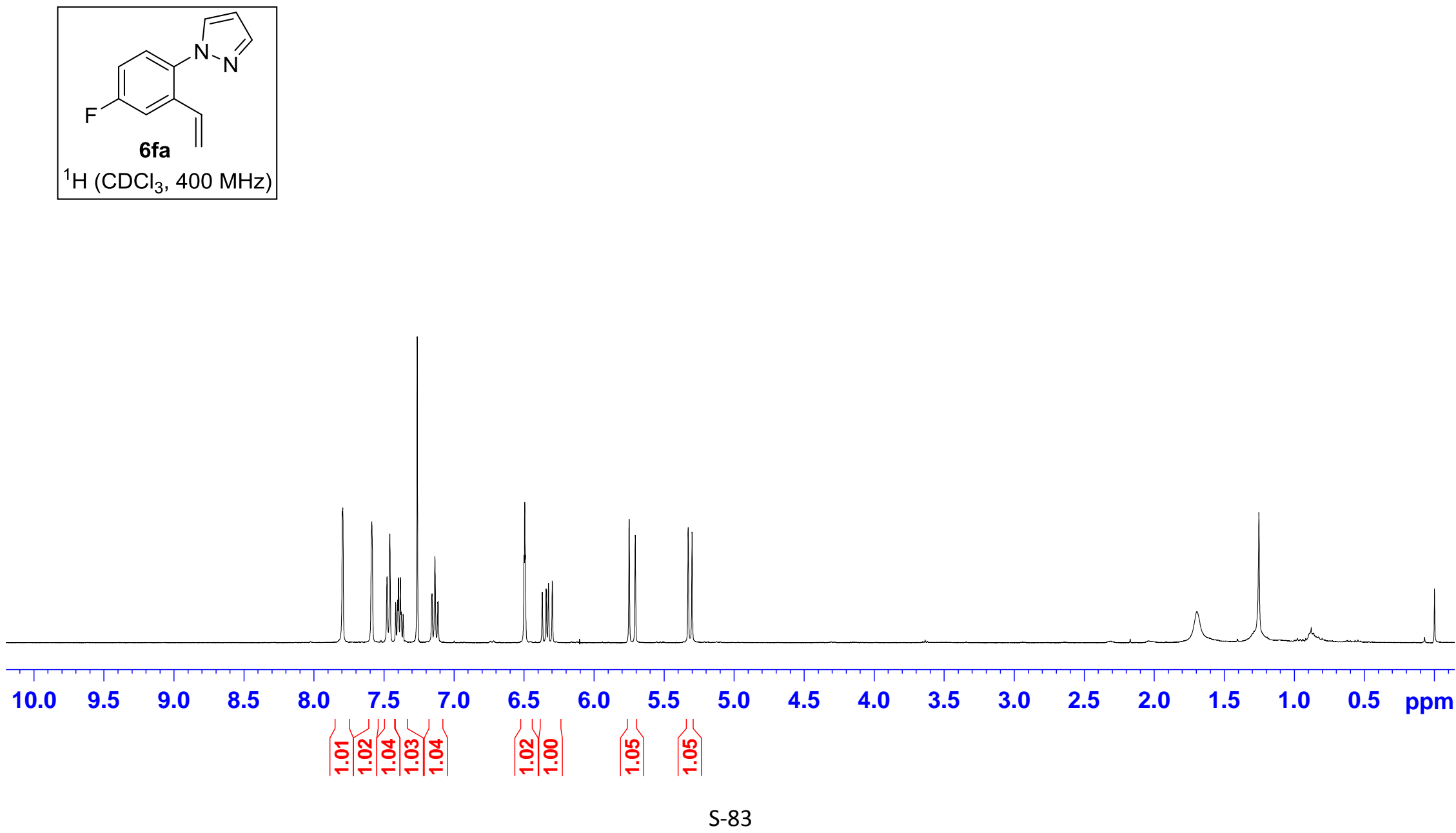


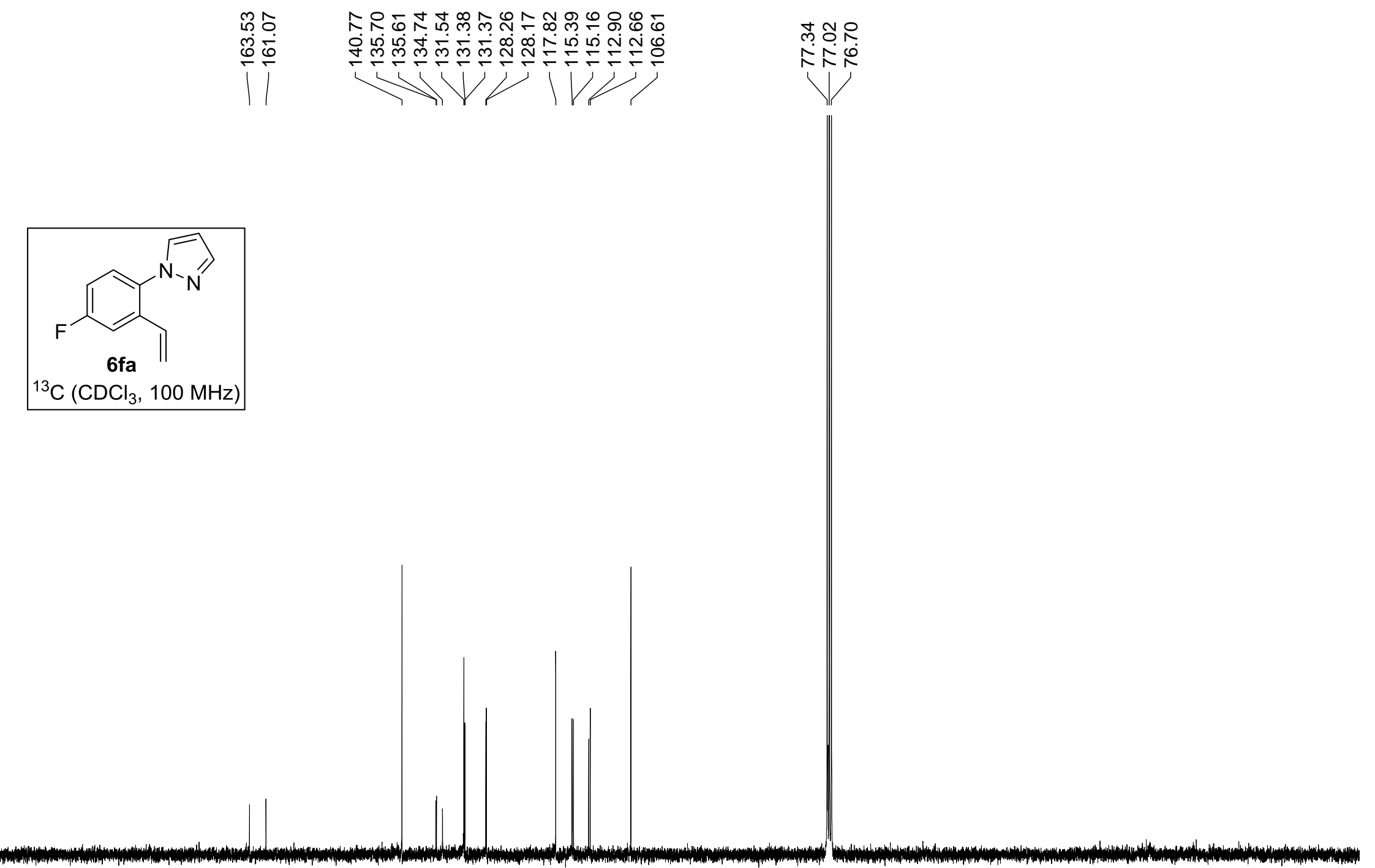

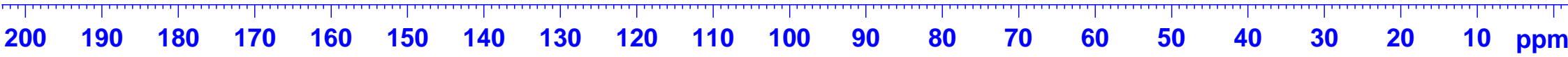



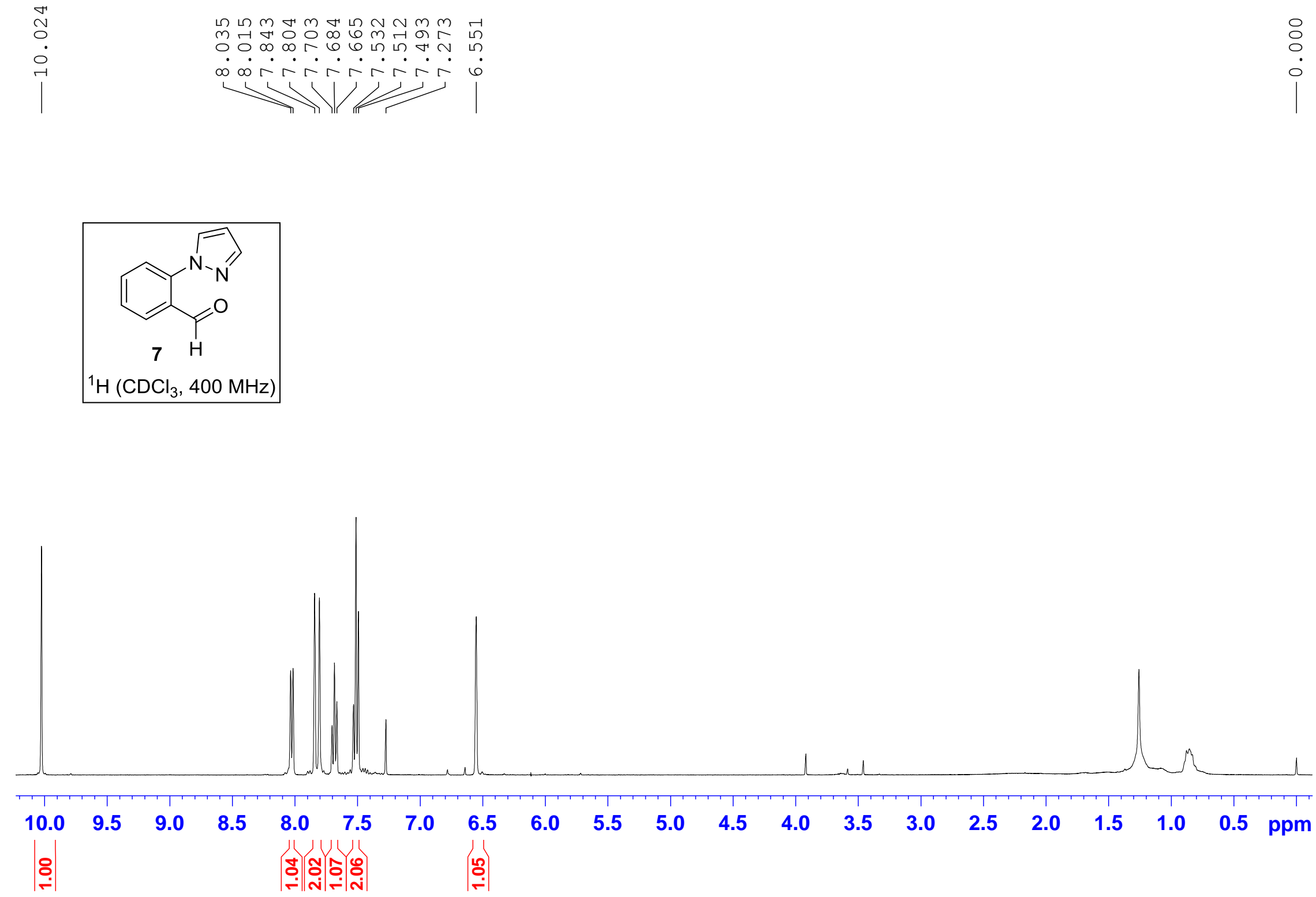


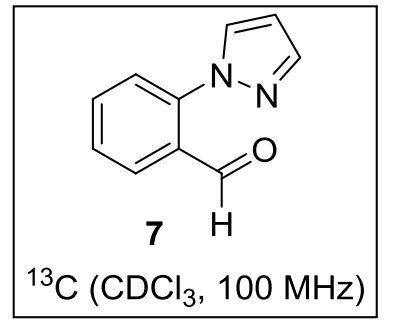

I.t.

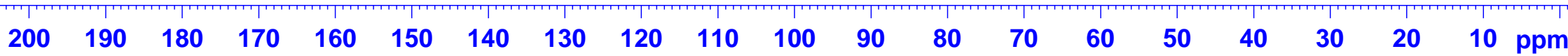

